\title{
Søren Buus. Thirty years of psychoacoustic inspiration
}

\section{Poulsen, Torben}

Published in:

Jounarl of Acoustical Society of America

Publication date:

2005

Document Version

Publisher's PDF, also known as Version of record

Link back to DTU Orbit

Citation (APA):

Poulsen, T. (2005). Søren Buus. Thirty years of psychoacoustic inspiration. In Jounarl of Acoustical Society of America (Vol. 117/4). Acoustical Society of America.

\section{General rights}

Copyright and moral rights for the publications made accessible in the public portal are retained by the authors and/or other copyright owners and it is a condition of accessing publications that users recognise and abide by the legal requirements associated with these rights.

- Users may download and print one copy of any publication from the public portal for the purpose of private study or research.

- You may not further distribute the material or use it for any profit-making activity or commercial gain

- You may freely distribute the URL identifying the publication in the public portal

If you believe that this document breaches copyright please contact us providing details, and we will remove access to the work immediately and investigate your claim. 


\title{
Session 2aAA
}

\section{Architectural Acoustics, Education in Acoustics, Noise and Psychological and Physiological Acoustics: Topical Meeting on Classroom Acoustics-The Research Perspective II}

\author{
Murray R. Hodgson, Cochair \\ Univ. of British Columbia, School of Occupational and Environmental Hygiene, 2206 East Mall, \\ Vancouver, BC V6T 1Z3, Canada \\ Lily M. Wang, Cochair \\ Univ. of Nebraska-Lincoln, Architectural Engineering, Peter Kiewit Institute, 1110 South 67th St., Omaha, NE 68182-0681
}

Chair's Introduction-8:00

Invited Papers

8:05

2aAA1. Survey of conditions in New Zealand primary school classrooms and research on mechanisms influencing speech perception for children. George Dodd (School of Architecture, Univ. of Auckland, Private Bag 92019, Auckland, New Zealand, g.dodd@auckland.ac.nz)

A survey of 122 classrooms in New Zealand supports the need for low reverberation time and low background noise if classrooms are to be satisfactory. The RT of "poor" and "good" classrooms was found to be $0.6 \mathrm{~s}$ and $0.4 \mathrm{~s}$ respectively. Six "poor" classrooms were modified to reduce their RT to $0.4 \mathrm{~s}$ which changed them to "good" as judged by the users. The need for this low RT may result from children having a small integration time. A novel technique of reversed-segmented speech has verified that this is significantly smaller for children compared with adults. Activity noise in classrooms exhibits the cafe effect-a rising noise level as children compete to be heard - and we suggest this is caused by the Lombard effect. Values of the Lombard effect that we measured in a cohort of primary school children predict classroom levels similar to those observed. Present theory does not predict that reducing the RT from 0.6 to $0.4 \mathrm{~s}$ should significantly influence the cafe effect. Further work is planned to refine the theory and to identify if there are mechanisms not accounted for.

\section{$8: 35$}

2aAA2. Auralization studies to develop a classroom questionnaire. Christian Nocke, Catja Hilge (Akustikbuero Oldenburg, Alte Raad 20a, D-26127 Oldenburg, Germany, info@akustikbuero-oldenburg.de), and Markus Meis (Hoerzentrum Oldenburg GmbH, D-26129 Oldenburg, Germany)

Modern, computer-based room acoustic modeling software allows apart from the calculation and visualization of sound fields also the auralization of room acoustic conditions. By these means the listening conditions in (virtual) class rooms can easily be modeled and modified. Furthermore, for each listening position a full set of room acoustical parameters is available. Corresponding measurements in real class rooms would require much effort; well-defined changes could only be reached by interior works in the building. So, computer modeling offers a larger variety of well-defined acoustic conditions. This contribution reports on the auralizations carried out for the development of a questionnaire for subjective evaluations in real class rooms. For this purpose six different virtual class rooms have been modeled. In each of the six situations auralizations at two different listening positions for male and female speakers have been deduced. The auralizations have been presented to 80 test persons. The objective room acoustic parameters have been correlated with the subjective judgements. The questionnaire has been used by Meis et al. in real life experiments on the subjective evaluation of listening conditions. Results of these experiments will be presented in another contribution to this session by M. Meis (2pAAa1).

\section{8:55}

2aAA3. Subjective and objective assessments in classrooms following acoustical renovation. Arianna Astolfi (Dept. of Energy Technologies, Politecnico di Torino, Corso Duca degli Abruzzi, 24, 10129, Torino, Italy)

The effectiveness of an expensive acoustical intervention in an old Italian high school building has been assessed in this work. The school building has fifty classrooms, the majority of which were acoustically renovated. A subjective survey and measurements were performed in both the renovated and non renovated classrooms. With the competence of some psychologists from Turin University, a questionnaire was set up for the subjective analysis. The questionnaire, validated after numerous pilot tests, was submitted to all the students and the teachers in two periods of the year. The questions on acoustical features included questions on annoyance from room noise, reverberation, speech comprehension, overall acoustical satisfaction and the consequences of bad acoustical conditions. Apart from the acoustics, other aspects of environmental quality, such as the thermal and visual environmental features and IAQ were investigated. The statistical analysis of the subjective answers allowed aggregated information to be obtained on the users and different data to be correlated. The aim of the statistical correlation was to determine any significant relationships between the objective and subjective data, and between the overall satisfaction scores and the different environmental factors. The effects of bad environmental conditions and their influence on learning capacity were also examined. 
2aAA4. Questionnaire study of perceived listening quality in renovated university classrooms. Murray Hodgson and Hugh Davies (UBC School of Occ. \& Env. Hygiene, 3rd Fl., 2206 East Mall, Vancouver, BC, Canada V6T 1Z3)

The research reported here investigated the effect of renovations on student perception of the listening environment in university classrooms. It involved four large classrooms at the University of British Columbia which were studied before and after acoustical renovation. Details of the renovations were identified. Measurements of reverberation time, background-noise level and sound propagation were made in each case. Room-average Speech Transmission Indices were calculated as a physically-based summary quality measure, and their changes on renovation were determined. Questionnaires designed to determine student and instructor perception of the listening environment, as well as the personal, academic and environmental factors that might affect it, were administered to a large number of students taking classes in each classroom, and their instructors, before and after renovation. A summary Perceived Listening Ease (PLE) score, measuring student perception of the quality of the listening environment, was calculated for each questionnaire from the responses. Room-average PLE scores, and the changes on renovation, were then calculated. These results were related to the physical measurement results. Physical, personal, academic and environmental factors affecting the changes in PLE score were investigated. The implications of the results for classroom design were considered.

\section{9:35}

2aAA5. Acoustical conditions for speech communication in active elementary school classrooms. Hiroshi Sato (Inst. for Human Sci. \& Biomed. Eng., Natl. Inst. of Adv. Industrial Sci. and Technol., 1-1-1 Higashi, Tsukuba, Japan) and John Bradley (Inst. for Res. in Construction Natl. Res. Council, Ottawa, Canada K1A 0R6)

Detailed acoustical measurements were made in 34 active elementary school classrooms with typical rectangular room shape in schools near Ottawa, Canada. There was an average of 21 students in classrooms. The measurements were made to obtain accurate indications of the acoustical quality of conditions for speech communication during actual teaching activities. Mean speech and noise levels were determined from the distribution of recorded sound levels and the average speech-to-noise ratio was $11 \mathrm{dBA}$. Measured mid-frequency reverberation times (RT) during the same occupied conditions varied from 0.3 to $0.6 \mathrm{~s}$, and were a little less than for the unoccupied rooms. RT values were not related to noise levels. Octave band speech and noise levels, useful-to-detrimental ratios, and Speech Transmission Index values were also determined. Key results included: (1) The average vocal effort of teachers corresponded to louder than Pearsons Raised voice level; (2) teachers increase their voice level to overcome ambient noise; (3) effective speech levels can be enhanced by up to $5 \mathrm{~dB}$ by early reflection energy; and (4) student activity is seen to be the dominant noise source, increasing average noise levels by up to $10 \mathrm{dBA}$ during teaching activities. [Work supported by CLLRnet.]

\section{9:55}

2aAA6. Investigation of noise issues at U-Hill Elementary School. Natalie York and Murray Hodgson (UBC Acoust. and Noise Res. Group, SOEH, 3rd Fl., 2206 East Mall, Vancouver, BC, Canada V6T 1Z3, nlyork@interchange.ubc.ca)

University Hill Elementary School (U-Hill) has a reputation amongst its teachers, staff and parents as being a noisy school. The present work is an assessment of the acoustical environment at U-Hill with a focus on evaluating how various noise sources contribute to the total noise levels measured in four classrooms. A questionnaire was completed by several of U-Hill's teachers to determine their reactions to the school's acoustical environments and what they perceived to be the prevalent noise sources. Standard physical measurements of classroom background noise and reverberation time were made and compared to existing acceptability criteria. Measurements of sound propagation and transmission were made to determine how noise propagates in the hallway, as well as into and between adjacent learning spaces, and the effect of open doors and door grates. Dosimeters were used to monitor sound levels at various hallway source locations and received in the four classrooms studied. Octave-band measurements of typical noise-source levels, such as children walking in the hallways and entering through school doors were made. The measurement data was used to estimate the contributions to total noise levels in the four classrooms of the various noise sources inside and outside the classrooms.

10:15-10:30 Break

\section{0:30}

2aAA7. Acoustical conditions of typical classrooms in Hong Kong. Kai Ming Li and Coriolanus C. L. Lam (Dept. of Mech. Eng., The Hong Kong Polytechnic Univ., Hung Hom, Hong Kong)

This paper presents measurement results of the acoustical environments of local schools in Hong Kong. In the measurements, several acoustical aspects that affect verbal communication in classrooms have been studied. These conditions include outdoor and indoor ambient noise levels, signal-to-noise ratios, reverberation time and the speech transmission index. Typical classrooms in many different schools and other higher-education institutions have been selected in the present study. Experimental results are compared with such national standards as USA (ANSI S 12.60 V 2002), Australian/New Zealand (AS/NZS 2107:2000), China (GB/T 15508 V 1995) and other national and industrial standards. This study will form the basis of devising acceptable standards for use in Hong Kong. [Work supported by the Research Grants Council of the SAR Government, the Research Committee of the Hong Kong Polytechnic University and Architectural Services Department of the Hong Kong SAR Government.] 
2aAA8. Acoustic and social design of schools-ways to improve the school listening environment. Mechthild Hagen (Education Dept., Ludwig-Maximilians-Univ. of Munich, Leopoldstr. 13, D-80802 Muenchen, Germany, m_hagen@ @rimedu.uni-muenchen.de)

Results of noise research indicate that communication, and as a result, teaching, learning and the social atmosphere are impeded by noise in schools. The development of strategies to reduce noise levels has often not been effective. A more promising approach seems to be to pro-actively support the ability to listen and to understand. The presentation describes the approach to an acoustic and social school design developed and explored within the project "GanzOhrSein" by the Education Department of the LudwigMaximilians-University of Munich. The scope includes an analysis of the current "school soundscape," an introduction to the concept of the project to improve individual listening abilities and the conditions for listening, as well as practical examples and relevant research results. We conclude that an acoustic school design should combine acoustic changes in classrooms with educational activities to support listening at schools and thus contribute to improving individual learning conditions and to reducing stress on both pupils and teachers.

\section{1:10}

2aAA9. New schools design: Acoustics as main target. Luigi Maffei and Paola Lembo (Dispama, Faculty of Architecture, Second Univ. of Naples, Via S. Lorenzo, 81031 Aversa (Ce), Italy)

The effects of poor intelligibility and high background noise levels on the cognitive development of school children and on the dissatisfaction of teachers has been largely investigated. National standards have been implemented and attempts to harmonize these standards in international guidelines are ongoing. All these activities have led to the awareness that design of new schools must be centered on the achievement of a good acoustic environment. At this point a strong research effort to study and implement best solutions must be conducted, in collaboration, by architects, acousticians, pedagogues, psychologists, builders and acoustic materials producers. Recently an international competition for the planning of new primary schools in Rome, Italy has been announced. The aim of the competition is to study new architectural and running features of primary schools to obtain, among other parameters such as lighting, low cost energy solutions and air quality, the control of reverberation time, sound insulation and mechanical equipments noise. In these school buildings, as innovative requirement, children must be also able to elaborate interpretative hypothesis of physical phenomena such as sound emission and perception and be aware of their influence on these phenomena. Different possible solutions are presented.

\section{Contributed Papers}

\section{$11: 30$}

2aAA10. Speech intelligibility metrics in small unoccupied classrooms. Matthew E. Cruikshank, Melinda J. Carney, and Dominique J. Cheenne (Dept. of Audio Arts \& Acoust., Columbia College Chicago, Chicago, IL 60605)

Nine small volume classrooms in schools located in the Chicago suburbs were tested to quantify speech intelligibility at various seat locations. Several popular intelligibility metrics were investigated, including Speech Transmission Index (STI), \%Alcons, Signal to Noise Ratios (SNR), and 80 ms Useful/Detrimental Ratios (U80). Incorrect STI values were experienced in high noise environments, while the U80s and the SNRs were found to be the most accurate methodologies. Test results are evaluated against the guidelines of ANSI S12.60-2002, and match the data from previous research.

\section{1:45}

2aAA11. The prediction of speech intelligibility in classrooms using computer models. Stephen Dance and Roger Dentoni (Dept. of Eng. Systems, London South Bank Univ., Borough Rd., London, UK)

Two classrooms were measured and modeled using the industry standard CATT model and the Web model CISM. Sound levels, reverberation times and speech intelligibility were predicted in these rooms using data for 7 octave bands. It was found that overall sound levels could be predicted to within $2 \mathrm{~dB}$ by both models. However, overall reverberation time was found to be accurately predicted by CATT $14 \%$ prediction error, but not by CISM, $41 \%$ prediction error. This compared to a $30 \%$ prediction error using classical theory. As for STI: CATT predicted within $11 \%$, CISM to within $3 \%$ and Sabine to within $28 \%$ of the measured value. It should be noted that CISM took approximately 15 seconds to calculate, while CATT took 15 minutes. CISM is freely available on-line at www.whyverne.co.uk/acoustics/Pages/cism/cism.html 


\title{
Session 2aAB
}

\section{Animal Bioacoustics: Methodology for Measurements of Auditory Evoked Potentials in Aquatic Mammals I}

\author{
Mardi C. Hastings, Chair \\ Office of Naval Research, ONR 341, 800 North Quincy St., Arlington, VA 22217 \\ Chair's Introduction-8:00 \\ Invited Papers
}

8:05

2aAB1. Conditions for evoked-potential audiometry in odontocetes. Alexander Ya. Supin (Inst. of Ecology and Evolution, Russian Acad. of Sci., 33 Leninsky Prospect, 119071 Moscow, Russia)

Currently auditory brainstem evoked potentials (ABR) become widely used for audiometry in odontocetes. Depending on the goal, the ABR technique differs with respect of (i) electrode positions, (ii) stimulus parameters, and (iii) threshold evaluation procedure. (I) Optimal electrode positions are vertex (for binaural responses), lateral (for monaural responses), or pharyngeal (for some express investigations). (II) The shorter the stimulus and the wider its spectrum, the more robust the response. Thereafter, narrow-band stimuli provoke low-amplitude ABR with a short dynamic range, but the goal of investigation often requires keeping the stimulus spectrum narrow. Sinusoidally amplitude modulated (SAM) sounds have narrow spectra, thereby provoking low amplitude envelope-following response (EFR); however, EFR can be extracted from noise by Fourier analysis. Modulation rate for SAM sounds must fit a spectrum peak of the ABR waveform. High-frequency sounds are more effective to provoke ABR and EFR than low-frequency ones, so ABR technique is better applicable for measurements in high-frequency ranges. (III) For precise threshold evaluation, low-amplitude responses must be extracted from noise. Apart from the averaging procedure, cross-correlation (for single ABR) or Fourier (for EFR) analysis is helpful to extract and measure low response amplitudes. [Work supported by Russian Basic Research Foundation, Russian President Grant, ONR.]

\section{8:35}

2aAB2. Auditory evoked potential measurement methodology for odontocetes and a comparison of measured thresholds with those obtained using psychophysical techniques. Paul E. Nachtigall, Michelle Yuen, T. Aran Mooney, and Kristen Taylor (Marine Mammal Res. Program, Hawaii Inst. of Marine Biol., Univ. of Hawaii, Honolulu, HI 96822)

Most measurements of the hearing capabilities of toothed whales and dolphins have been taken using traditional psychophysical procedures in which the animals have been maintained in laboratory environments and trained to behaviorally report the sensation or difference of acoustic stimuli. Because of the advantage of rapid data collection, increased opportunities, and new methods, Auditory Evoked Potentials (AEPs) have become increasingly used to measure audition. The use of this new procedure calls to question the comparability of the established literature and the new results collected with AEPs. The results of behavioral and AEP methods have been directly compared with basic audiogram measurements and have been shown to produce similar (but not exactly the same) values when the envelope following response procedure has been used and the length of the stimulus is taken into account. The AEP methods allow possible audiometric opportunities beyond those available with conventional psychophysics including: (1) the measurement of stranded dolphins and whales that may never be kept in laboratories, (2) the testing of stranded animals for hearing deficits perhaps caused by overexposure to noise, and (3) passive testing of hearing mechanisms while animals actively echolocate. [Work supported by the Office of Naval Research and NOAA-NMFS.]

\section{9:00}

2aAB3. Auditory evoked potential measurements with cetaceans. David Mann, Mandy Cook (USF College of Marine Sci. \& Mote Marine Lab., 140 7th Ave. S., St. Petersburg, FL 33701, dmann@ marine.usf.edu), Gordon Bauer (New College of Florida \& Mote Marine Lab., Sarasota, FL 34243), Wendi Fellner (Walt Disney World, Lake Buena Vista, FL), and Randy Wells (Chicago Zoological Society and Mote Marine Lab., Sarasota, FL 34236)

Auditory evoked potentials (AEPs) allow researchers to measure the hearing abilities of animals that would be difficult or impossible to train for behavioral measurements of hearing. The hearing abilities of live-stranded cetaceans and wild dolphins can only be made with AEP techniques. In these situations, time with the animal is often restricted to an hour or less, and there is often little control over the acoustic environment in which the tests are performed. AEP measurements may be made while the animals are in air or in shallow pools. For cetaceans in air, sounds are typically presented with a suction cup jawphone. For cetaceans in water, sounds may be presented in a direct field (with the transducer located at some distance from the test subject) or with a jawphone. In each of these situations it is important to understand how thresholds derived from AEP measurements compare with behavioral hearing measurements. Examples of AEP measurements from wild and live-stranded cetaceans are presented to illustrate their usefulness and the constraints under which these measurements must be made. AEP measurements from bottlenose dolphins in air and in water are also compared with their behavioral audiograms. 
2aAB4. Narrow band pulses as stimuli in an auditory brain stem recording study with a harbor porpoise. Kristian Beedholm and Lee A. Miller (Inst. of Biol., Univ. of Southern Denmark, Campusvej 55, DK-5230 Odense M, Denmark, lee@ biology.sdu.dk)

We have studied several aspects of hearing by a harbor porpoise using the ABR method with pulsed stimuli. Experiments were conducted on a male porpoise in collaboration with Fjord and Baelt, Kerteminde, Denmark. The animal had suction cups containing silver electrodes placed near the blowhole and near the dorsal fin. When fitted with the electrodes he moved to an underwater listening post where his outgoing sonar signal could be used to trigger a phantom echo. EEG signals were amplified differentially and averaged over a variable number of presentations depending on trial duration and experiment. For studying the frequency/intensity response, narrow band pulsed stimuli were generated and presented in several ways. One way was to use the impulse response of a B\&K $1 / 3$ octave filter bank (set to $80,100,125$, or $160 \mathrm{kHz}$ ) as a stimulus. This stimulus was presented in both a passive hearing task, when a signal generator triggered the echo, and in an active experiment, where the echo was time locked to the animals emitted signal. Our results show the best response at $125 \mathrm{kHz}$ and indicate a slight, but significantly higher response in the active mode. The latter has a methodological explanation. [Work supported by ONR.]

9:50

2aAB5. Auditory evoked potentials (AEP) methods for population-level assessment of hearing sensitivity in bottlenose dolphins. Dorian Houser (BIOMIMETICA, 7951 Shantung Dr., Santee, CA 92071) and James Finneran (Space and Naval Warfare Systems Ctr., San Diego, CA 92152)

A portable system for recording auditory evoked potentials (AEP) was developed to rapidly assess the hearing sensitivity of dolphins in air. The system utilizes a transducer embedded in a silicone suction cup to deliver amplitude modulated tones to the dolphin through the lower jaw. Frequencies tested range from $10-150 \mathrm{kHz}$ and testing of both ears is completed within $90 \mathrm{~min}$. AEP-determined thresholds from one subject were benchmarked against that subject's direct field behavioral audiogram to quantify variation between the two methods. To date, AEP audiograms have been obtained from over 30 bottlenose dolphins. Considerable individual variation in frequency-specific hearing sensitivity was observed. Some high-frequency hearing loss was observed in relatively young (early 20s) and old (35+ years) animals; conversely, age was not necessarily related to hearing loss as several animals greater than 40 years of age had good hearing sensitivity across the range of tested frequencies. Profound hearing loss typically occurred at higher frequencies. Decline in sensitivity was rapid in all cases and began between $50-60 \mathrm{kHz}$. Increased sample size of hearing sensitivity in dolphins suggest that the use of audiometric functions from single animals as representative of population level audiometry might be misleading.

\section{0:15-10:30 Break}

\section{0:30}

2aAB6. Electrophysiological methods for hearing assessment in pinnipeds. Colleen Reichmuth Kastak, David Kastak (Univ. of California Santa Cruz, Long Marine Lab., 100 Shaffer Rd., Santa Cruz, CA 95060, coll@ucsc.edu), James J. Finneran (SPAWARSYSCEN San Diego, San Diego, CA 92152), Dorian S. Houser (BIOMIMETICA, Santee, CA 92071), and Alexander Supin (Inst. of Ecology and Evolution, 119071 Moscow, Russia)

Studies of auditory sensitivity in marine mammals generally rely on behavioral psychophysical methodologies. While these studies are the standard for hearing assessment in marine mammals, data are limited to only a few individuals representing a small proportion of species. Accumulating research on dolphin auditory physiology has resulted in the refinement of electrophysiological methods appropriate for odontocete cetaceans and an increase in available audiometric information. Electrophysiological methods have also been used with pinnipeds, but there are significant gaps in our understanding of pinniped auditory physiology that must be addressed before such appoaches can be broadly applied to investigations of pinniped hearing. We are taking a bottom-up approach to developing suitable methods for evoked potential audiometry in pinnipeds, including technology transfer from studies of cetaceans and other mammals, mapping of response amplitude with respect to recording positions on the skull, characterization of responses in relationship to various stimulus types and presentation parameters, and determination of whether useful frequency-specific data can be reliably obtained using electrophysiological methods. This approach is being taken with representative pinniped species including California sea lions (Zalophus californianus), harbor seals (Phoca vitulina), and northern elephant seals (Mirounga angustirostris) using both training and chemical immobilization techniques. [Work supported by NOPP.]

\section{0:55}

2aAB7. Ground-truthing evoked potential measurements against behavioral conditioning in the goldfish, Carassius auratus. Randy J. Hill (College of Marine Sci., Univ. of South Florida, 140 7th Ave. S., St. Petersburg, FL 33701) and David A. Mann (Univ. of South Florida, St. Petersburg, FL 33701)

Auditory evoked potentials (AEPs) have become commonly used to measure hearing thresholds in fish. However, it is uncertain how well AEP thresholds match behavioral hearing thresholds and what effect variability in electrode placement has on AEPs. In the first experiment, the effect of electrode placement on AEPs was determined by simultaneously recording AEPs from four locations on each of 12 goldfish, Carassius auratus. In the second experiment, the hearing sensitivity of 12 goldfish was measured using both classical conditioning and AEP's in the same setup. For behavioral conditioning, the fish were trained to reduce their respiration rate in response to a $5 \mathrm{~s}$ sound presentation paired with a brief shock. A modified staircase method was used in which 20 reversals were completed for each frequency, and threshold levels were determined by averaging the last 12 reversals. Once the behavioral audiogram was completed, the AEP measurements were made without moving the fish. The recording electrode was located subdermally over the medulla, and was inserted prior to classical conditioning to minimize handling of animal. The same sound stimuli (pulsed tones) were presented and the resultant evoked potentials were recorded for 1000-6000 averages. AEP input-output functions were then compared to the behavioral audiogram to compare techniques for estimating behavioral thresholds from AEP data. 
2aAB8. Electrophysiological responses to vibratory stimulation in the brainstem of the tadpole. Andrea M. Simmons, Aimee Two, and Seth S. Horowitz (Dept. Psych. and Neurosci., Brown Univ., Providence, RI 02912, Andrea_Simmons@brown.edu)

Maturation of saccular responses to vibratory stimuli in 3 axes was measured in the Rana catesbeiana tadpole across metamorphic development. The animal was suspended in a water-filled circular aluminum dish attached to a shaker table system consisting of 5 vibration exciters producing sinusoidal, translational movements in the $x, y$, and $z$ planes. Multi-unit responses were recorded from the medulla with tungsten or platinum-iridium microelectrodes. Recording sites were electrolytically marked to verify electrode placement. Data were collected from 16 animals in late larval stages, before onset of metamorphic climax. Most recording sites were sensitive to vibrations in the $z$ (vertical) direction, indicating a saccular origin of the response. Vibration frequencies of $40 \mathrm{~Hz}$ or below produced the strongest activity, with thresholds below a displacement of $10 \mathrm{~nm}$. Secondary sensitivity was sometimes observed to either $x$ (body-longitudinal) or $y$ (body-lateral) directions of movement. Although some sites produced strong phase-locked activity to the stimulation, others showed more diverse temporal patterns of response. Active recording sites were found in the medial vestibular nucleus, lateral vestibular nucleus, reticular gray, and dorsal superior olivary nucleus. These data suggest that tadpole saccule is functional early in larval development, with sensitivity similar to that observed in adult bullfrogs.

TUESDAY MORNING, 17 MAY 2005

PLAZA A, 9:00 TO 11:45 A.M.

\title{
Session 2aAO
}

\section{Acoustical Oceanography, Underwater Acoustics and Animal Bioacoustics: Acoustics and Ocean Observatories I}

\author{
Bruce M. Howe, Chair \\ Applied Physics Lab., Univ. of Washington, 1013 NE 40th St., Seattle, WA 98105-6698
}

Chair's Introduction-9:00

Invited Papers

9:05

2aAO1. Initial results from the Pacific Ocean Shelf Tracking System: A permanent continental-scale acoustic tracking array for fisheries research. David W. Welch (Chief Scientist, Census of Marine Life Project POST, Kintama Res. Corp., 4737 Vista View Crescent, Nanaimo, BC, Canada V9V 1N8)

The Census of Marine Life is developing POST, a seabed acoustic array for tracking marine animals. The long-range plan involves the deployment of 30 or more cross-shelf monitoring lines forming a permanent continental-scale array, each consisting of autonomous seabed nodes spaced at roughly $1 \mathrm{~km}$ intervals. Nodes would be modular and use an acoustic modem to periodically communicate with an overhead ship, which would upload data and download new programming. 2004 was a large-scale test of the tracking array component using $135 \mathrm{~km}$ of listening lines and 1050 acoustically tagged juvenile salmon (12-15 cm long). Detection rates for individual fish crossing $20 \mathrm{~km}$ long acoustic lines were approximately $91 \%$, and precise measurements of migration timing, speed of movement and survival were obtained for both the freshwater and early marine phases. In subsequent years we plan to include additional oceanographic sensors to provide detailed data on changes in bottom temperature, salinity, and currents over time and implement acoustic modems to allow remote data upload from a visiting boat. These data could then be meshed with the fish movement data to describe how animals move relative to changes in the three dimensional structure of the ocean that they are migrating through.

9:25

2aAO2. Autonomous underwater vehicle networks as integrated acoustic observation systems. Henrik Schmidt (MIT, 77 Massachusetts Ave., Cambridge, MA 02139)

Autonomous Underwater Vehicles (AUV) are rapidly being transitioned into operational systems for national defense, offshore exploration, and ocean science. However, the use the robotic platforms as components of integrated acoustic sensing systems is still at an early stage. Thus, for example, with recent advances in underwater navigation and communication, and collaborative robotics, networks of AUVs may have significant potential as moving tomographic networks for optimally and adaptively providing in-situ environmental estimates. Such concepts are important to the development and operation of environmenatally adaptive, autonomous littoral surveillance systems by providing environmental inversion specifically emphasizing the parameters significant for the actual platform configuration. Also such multi-AUV concepts have significant potential for subbottom characterization in the deep ocean, of increased importance in the offshore industry. For such systems to be operationally feasible, the limited underwater communication bandwidth makes it necessary for such systems to involve significant on-board processing, in turn requiring the acoustic sensing to be fully integrated with an onboard modeling capability and the platform navigation and control. The development of such new nested, distributed processing concepts, autonomously integrating sensing, modeling and control, is a significant challenge to the acoustics community. [Work supported by ONR.] 
2aA03. Communications performance of an undersea acoustic large-area network. Hannah A. Kriewaldt (Phys. Dept., Naval Postgrad. School, Monterey, CA 93943, hakessle@nps.edu) and Joseph A. Rice (SSC San Diego, Naval Postgrad. School, Monterey, CA 93943)

The U.S. Navy is developing Seaweb acoustic networking capability for integrating undersea systems. Seaweb architectures generally involve a wide-area network of fixed nodes consistent with future distributed autonomous sensors on the seafloor. Mobile nodes including autonomous undersea vehicles (AUVs) and submarines operate in the context of the grid by using the fixed nodes as both navigation reference points and communication access points. In October and November 2004, Theater Anti-Submarine Warfare Exercise (TASWEX04) showcased Seaweb in its first fleet appearance. This paper evaluates the TASWEX04 Seaweb performance in support of networked communications between a submarine and a surface ship. Considerations include physical-layer dependencies on the $9-14 \mathrm{kHz}$ acoustic channel, such as refraction, wind-induced ambient noise, and submarine aspect angle. [Work supported by SSC San Diego.]

\section{0:05-10:25 Break}

\section{$10: 25$}

2aAO4. Acoustic navigation for ultra-precise seafloor mapping. Donald M. Hussong and J. Grant Blackinton (Fugro Seafloor Surveys, Inc., 2727 Alaskan Way, Seattle, WA 98121)

Numerous autonomous underwater vehicles (AUVs) are operating with very precise swath bathymetric mapping systems in ocean depths to $3000 \mathrm{~m}$ and greater. Although these systems acquire data from positions known to a few centimeters accuracy with respect to the vehicle position, the positioning of the AUVs is generally no better than tens of meters at great depths. Although not yet assembled into an operational system, the technology now exists to acoustically navigate an AUV to within a $10 \mathrm{~cm}$ in $x, y$, and $z$. This begins by establishing active reference positions on the seafloor, known to within a few centimeters absolutely, using acoustic mirror transponders that transmit and receive streams of pulse coded signals from an accurately navigated surface vessel. These bottom reference positions can then be the core of a long baseline acoustic navigation system which, when coupled with constant monitoring of oceanographic conditions to calculate pressure corrections for depth measurements, will provide 10-cm navigation accuracy for the mapping AUV. Implementation of this technology will enable observational mapping of highly seismic areas where frequent earthquakes can cause many meters of deformation of the seafloor, and can generate massive tsunamis.

\section{Contributed Papers}

\section{0:45}

2aAO5. NEPTUNE Canada-status and planning. Brian D. Bornhold (NEPTUNE Canada, P.O. Box 1700, STN CSC, Univ. of Victoria, Victoria, BC, Canada V8W 2Y2, bornhold@uvic.ca)

Stage 1 of the joint Canada-U.S. NEPTUNE seafloor observatory has been funded by the Canada Foundation for Innovation and the British Columbia Knowledge Development Fund with an overall budget of $\$ 62.4$ million. The network is designed to provide as close to real-time data and images as possible to be distributed to the research community, government agencies, educational institutions and the public via the Internet. Covering much of the northern segment of the Juan de Fuca Plate, this first phase of the NEPTUNE project is scheduled to be installed, with an initial suite of "community experiments", in 2008. As part of the planning, NEPTUNE Canada held a series of three workshops to develop the science plans for these "community experiments"; these experiments have a budget of approximately $\$ 13$ million. The experiments will cover the gamut of oceanographic science themes including various aspects of: ocean climate and marine productivity, seabed environments and biological communities, fluids at ocean ridges, gas hydrates and fluids on continental margins, plate tectonics processes, associated earthquakes and tsunamis. The next three years will be spent developing and testing the necessary instrumentation for deployment on the network.

\section{1:00}

2aAO6. Ambient sound budgets. Jeffrey A. Nystuen and Bruce M. Howe (Appl. Phys. Lab., 1013 NE 40th St., Seattle, WA 98105)

The underwater ambient sound field contains a lot of information about geophysical, biological and anthropogenic activities in the ocean. Identification and quantification of these signals will complement more traditional measurements collected on future ocean observatories, including systems cabled to shore and remote mooring arrays. Long-term ambient sound measurements using a low duty cycle recorder are reported from remote deep ocean moorings, coastal shelf moorings, inland waterways and even a deep, quiet fresh water lake. Identifiable geophysical signals include accurate measurements of wind speed and rainfall over a wide range of wind speeds and rainfall rates. The character and temporal patterns of biological activity, shipping and recreational boating are monitored. The overall ambient sound budgets will be reported for these different environments. Acoustic monitoring is available from sub-surface moorings, remote locations and under severe weather conditions where and when other ocean instrumentation is likely to fail. [Work supported by ONR Ocean Acoustics.]

\section{1:15}

2aA07. The role of ocean observatories in monitoring for potential effects of man-made sound on the marine environment. David L. Bradley (Appl. Res. Lab., Penn State Univ., P.O. Box 30, State College, PA 16804), Gerald L. D'Spain (Scripps Inst. of Oceanogr., La Jolla, CA 93940-0701), James H. Miller (Univ. of Rhode Island, Narragansett, RI 02882), and George V. Frisk (Florida Atlantic Univ., Dania Beach, FL 33004-3023)

An important question is whether or not man-made sounds have an adverse long-term impact on the ocean environment. Clear evidence exists that an impact has occurred on some individual animals in a few cases. However, according to the 2003 National Academy of Sciences report, the degree of impact on any marine mammal population or on ocean ecosystems as a whole is unknown. An essential component of an approach to addressing this question is an ocean noise/marine ecosystem monitoring system. The monitoring regions should be global in extent and include biologically sensitive areas. The effort should be sustained so that longterm trends in ocean noise levels can be evaluated. An important aspect of the data collection effort is the type and quality of ancillary information. Ancillary data on the marine ecosystems allows determination of any impacts and data on the sources of marine noise and marine environmental properties which are required to develop metrics for evaluating and predicting the characteristics of the noise field. The ocean observatory system presently being contemplated could play an important role in establishing 
an ocean noise monitoring capability. [Work supported through the National Ocean Partnership Program, with sponsorship from ONR, NOAA, NSF, and USGS.]

$$
11: 30
$$

2aA08. A nearshore observatory for Antarctic krill: Analysis of the spatial variability in their distribution and abundance. Joseph D. Warren (Southampton College, 239 Montauk Hwy, Southampton, NY 11968, joe.warren@liu.edu) and David A. Demer (Southwest Fisheries Sci. Ctr., La Jolla, CA 92037)

Annual surveys of the distribution and abundance of Antarctic krill are conducted by the United States Antarctic Marine Living Resources Program in order to assist fishery managers in conserving this economic and ecologic resource. These surveys utilize a large vessel which does not sample close (within $10 \mathrm{~km}$ ) to the South Shetland Islands. These islands are home to many krill predators (fur seals and penguins) who often forage in these nearshore waters. In order to better understand the abundance and distribution of the krill in these waters and to determine the physical and biological factors affecting the krill, a nearshore observatory has been created that combines multiple sensors and platforms to provide improved spatial and temporal coverage of the nearshore region of Livingston Island. This study utilizes a large research vessel, a small inflatable vessel, an array of instrumented buoys, and a pilot project involving an Autonomous Underwater Vehicle. This presentation will discuss acoustic backscatter measurements made from both large and small vessels. Results from the most recent field season (Jan-Feb 2005) will be presented as well as data from previous years $(2002,2004)$. [Work supported by the NSF Office of Polar Programs and NOAA.] \\ Session 2aBB \\ Biomedical Ultrasound/Bioresponse to Vibration and Physical Acoustics: Diagnostic and Therapeutic Ultrasound Relevant to the Brain
}

\author{
Pierre D. Mourad, Cochair \\ Applied Physics Lab., Univ. of Washington, Neurosurgery, 1013 NE 40th St., Seattle, WA 98195 \\ E. Carr Everbach, Cochair \\ Dept. of Engineering, Swarthmore College, 500 College Ave., Swarthmore, PA 19081-1397
}

\section{Invited Papers}

\section{8:00}

2aBB1. Opening the blood-brain barrier with ultrasound. Pierre Mourad (APL/Neurosurgery, Univ. of Washington, 1013 NE 40th St., Seattle, WA 98105, pierre@apl.washington.edu)

The blood-brain barrier (BBB) constitutes a unique anatomical structure within the brain that prevents many therapeutic chemicals within the blood from entering the brain. Research at the University of Washington has shown that it is possible to transiently disrupt the BBB without damage in an intra-operative setting, as verified by the flux of vital dyes from the blood-stream into the brain. This talk will review the research we have done to date on this subject, and discuss potential applications of this approach to BBB disruption.

\section{8:20}

2aBB2. Feasibility of noninvasive ultrasound delivery for tumor ablation and targeted drug delivery in the brain. Kullervo Hynynen, Nathan McDannold, Greg Clement, Jason White (Dept. of Radiol., Brigham and Women's Hospital, 75 Francis St., Boston, MA 02115), Lisa Treat, Xiangtao Yin, Ferenc Jolesz, Nickolai Sheikov (Brigham and Women's Hospital, Boston, MA 02115), and Natalia Vykhodtseva (Brigham and Women's Hospital, Boston, MA 02115)

The objective of our research during the past few years has been to develop multichannel ultrasound phased arrays for noninvasive brain interventions. We have been successful in developing methods for correcting the skull induced beam distortions and thus, are able to produce sharp focusing through human skulls. This method is now being tested for thermal ablation of tumors, with results from animal studies demonstrating feasibility. In addition, the ability of ultrasound to open the blood-brain barrier (BBB) locally has been explored in animal models. The results suggest that the transcranial ultrasound exposures can induce BBB opening such that therapeutic agents can be localized in the brain. This tool is especially powerful since the beam can be guided by MR images, thus providing anatomical or functional targeting. This talk will review our current status in this research, which ultimately aims for the clinical use of this methodology.

\section{$8: 40$}

2aBB3. Transcranial power $\boldsymbol{M}$-mode Doppler ultrasound for diagnosis of patent foramen ovale. Mark Moehring and Merrill Spencer (Spencer Technologies, 701 16th Ave., Seattle, WA 98122, mm@spencertechnologies.com)

Patent foramen ovale (PFO) is a right-to-left shunt (RLS) which communicates blood from the right to left atrium of the heart. PFO has been associated with stroke and, more recently, with migraine headache. Diagnosis of RLS can be accomplished effectively with transcranial power $M$-mode Doppler ultrasound (PMD). PMD is a modality which can be performed without the sedation 
required by the more invasive diagnostic technique using transesophageal echocardiography. PMD for this application consists of 2 $\mathrm{MHz}$ pulse Doppler ultrasound with placement of sample gates at $2 \mathrm{~mm}$ intervals along the single-transducer beam axis, and $8 \mathrm{kHz}$ pulse repetition rate (PMD100M, Spencer Technologies). Doppler power versus depth is constructed every 4ms, using 33 sample gates. Bubble microemboli injected in the venous system and moving across a PFO present as high intensity tracks on a PMD image, as emboli transit from the heart to the brain and through the observed cerebral vasculature. Use of PMD in this context has been reported in the clinical literature [M. P. Spencer, M. A. Moehring, J. Jesurum et al, J. Neuroimaging 14, 342-349 (2004)]. This talk surveys the basic technical features of PMD for sensing PFO-related showers of bubble microemboli, and how these features provide clues to the severity of PFO.

\section{Contributed Papers}

9:00

2aBB4. Frequency-dependent ultrasound transmission through the human skull. P. Jason White, G. T. Clement, and Kullervo Hynynen (Dept. of Radiol., Brigham and Women's Hospital, Harvard Med. School, Boston, MA 02115)

The development of large-aperture multiple-source transducer arrays for ultrasound transmission through the human skull has demonstrated the possibility of controlled acoustic energy delivery into the brain parenchyma. The individual control of acoustic parameters from each ultrasound source allows for the correction of distortions arising from transmission through the skull bone and also opens up the possibility for electronic steering of the acoustic focus within the brain. To determine the efficacy of frequency modulation with such a device, this study examines the frequency dependence of ultrasound transmission in the range of 0.61.4 MHz through a series of seven points on each of three ex vivo human calvaria. Using broadband pulses, it is shown that the reflected spectra from the skull reveal information regarding the transmission energies at specific frequencies. In fact, there exists an inverse correlation between the reflected pressure amplitude and the transmitted pressure amplitude such that, for the frequency range under examination, approximately three local minima in the reflection spectra with the corresponding three peaks in transmission are observed. A noninvasive determination of the most efficient transmission frequency for a localized spatial region on a skull can yield an improvement of up to $3.5-\mathrm{dB}$ in ultrasound intensity transmission.
9:15

2aBB5. Numerical simulation of ultrasound thermotherapy of brain with a scanned focus transducer. Sohrab Behnia, Farzan Ghalichi, Amin Jafari, and Ashkan Bonabi (Dept. of physics, IAU, Univ. of Ourmi, Iran, s.behnia@iaurmia.ac.ir)

Brain tumors are one of the most difficult ones to treat. The margin between destruction of the tumor and damage to the surrounding tissue is narrow in the brain. Ultrasound could be an effective treatment because of its ability to propagate deep in tissue and induce temperature rise at the focus while leaving the surrounding tissue intact. This study investigates whether using a fix-focus transducer could destroy brain tumor cells, in a cost effective manner which reduces the treatment time significantly. In this work an appropriate fix-focus transducer was designed considering effective parameters and limitations which are dominant in this case. Then a real 2-D brain model was constructed from a MR image. A piece of the skull bone has been removed to allow ultrasound to propagate into the brain. The resultant pressure field and the temperature rise were calculated by Rayleigh integral and bio-heat equation on the model. The obtained results were promising indicating that toxic temperatures could be obtained in short treatment times. This could be of great advantage especially in treating primary brain tumors.

\section{Invited Papers}

\section{9:30}

2aBB6. Ultrasound-accelerated thrombolysis using microbubbles. William Culp (Interventional Radiol., Univ. of Arkansas for Medical Sci., Little Rock, AR 72205-7199, CulpWilliamC@uams.edu)

Current thrombolytic therapy for ischemic stroke reaches less than $3 \%$ of cases and is only moderately successful. Numerous studies have demonstrated increased thrombolytic activity when ultrasound is delivered to clot in the presence of tissue plasminogen activator. Initial human reports using trans-cranial Doppler technology $(2 \mathrm{MHz})$ to deliver continuous ultrasound to intracranial clot are very promising with improved clot lysis and no increase in symptomatic bleeding. However, another human study using lowfrequency therapeutic ultrasound resulted in excessive bleeding and was discontinued. Microbubble augmented ultrasound clot lysis has proven successful in several studies ranging from lysis of very small peripheral clots in rabbits to very large clots in dialysis grafts in dogs. Early human studies of thrombosed dialysis grafts show success and no adverse events. Intracranial clot lysis in pigs has been successful with both intra-arterial and intravenous microbubble techniques. Better definition of possible combination therapies and of efficacy and safety is still required in animal models, but human studies should be designed to avoid some of the complications of current therapy. This presentation will review the subject, present recent advances, and define some requirements for successful clot destruction in peripheral vessels and intracranial vessels.

9:50

2aBB7. Ultrasound enhanced thrombolysis: Clinical evidence. Andrei V. Alexandrov (The Univ. of Texas-Houston Med. School, MSB 7.044, 6431 Fannin St., Houston, TX 77030)

Phase II CLOTBUST randomized clinical trial (Houston, Barcelona, Edmonton, Calgary) evaluated patients with acute ischemic stroke due to intracranial occlusion and treated with intravenous tissue plasminogen activator (TPA) within $3 \mathrm{~h}$ of symptom onset. Randomization: monitoring with pulsed wave $2 \mathrm{MHz}$ transcranial Doppler (TCD) (Target) or placebo monitoring (Control). Safety: symptomatic bleeding to the brain (sICH). Primary end-point: complete recanalization on TCD or dramatic clinical recovery by the total NIHSS score $<3$, or improvement by $>10$ NIHSS points within 2 hours after TPA bolus. All projected 126 patients were randomized 1:1 to target (median NIHSS 16) or control (NIHSS 17). sICH: $4.8 \%$ Target, $4.8 \%$ Controls. Primary end-point was achieved by 31 (49\%, Target) versus 19 (30\%, Control), $p<0.03$. At 3 months, 22 (42\% Target) and 14 (29\% Control) patients achieved favorable outcomes. Continuous TCD monitoring of intracranial occlusion safely augments TPA-induced arterial recanalization, and $2 \mathrm{MHz}$ diagnostic ultrasound has a positive biological activity that aids systemic thrombolytic therapy. For the first time in clinical medicine, the CLOTBUST trial provides the evidence that ultrasound enhances thrombolytic activity of a drug in humans thereby confirming intense multi-disciplinary experimental research conducted worldwide for the past 30 years. 
2aBB8. The effect of $40 \mathrm{kHz}$ ultrasound on tissue plasminogen activator-induced clot lysis in three in vitro models. Marlien Pieters, Rob T. Hekkenberg, Marrie Barrett-Bergshoeff, and Dingeman C. Rijken (TNO Prevention and Health, Leiden, Netherlands)

In previous work from the same laboratory, high-frequency ultrasound (US) (3 MHz) was shown to promote in vitro fibrinolysis through enhanced supply of plasminogen to the clot surface. The application of high-frequency US is limited in vivo due to tissue heating. Low-frequency US, however, has less tissue heating and improved penetration. Internal plasma clot lysis and external lysis with compacted and non-compacted plasma clots were used to determine the magnitude of the effect of low-frequency US (40 kHz; $0.5 \mathrm{~W} / \mathrm{cm} 2$ ) on tissue plasminogen activator-induced lysis and to elucidate the mechanisms behind the effect. Ultrasound enhanced lysis in all three models, with the largest effects (4-fold) in the external lysis model with compacted plasminogen-poor clots. The acceleration effect of ultrasound in this model decreased with increasing $t$-PA—and decreasing plasminogen concentrations. Ultrasound had a much smaller effect in this model when compacted plasminogen-rich clots were used. In the external lysis, noncompacted clot model, ultrasound resulted in consistently higher lysis rates. The acceleration effect of lysis, increased slightly (1.3 to 1.8 -fold) with increasing $t$-PA — and decreasing plasminogen concentrations. Plasminogen supply to the clot surface was again shown to be an important contributor to ultrasound-enhanced lysis. [M. Pieters, R. T. Hekkenberg, M. Barrett-Bergshoeff, and D. C. Rijken. Ultrasound in Med \& Biol 30, 1545-1552 (2004).]

\section{Contributed Papers}

\section{0:50}

2aBB9. Confocal microscopy movies of fibrin clots during ultrasoundaccelerated thrombolysis. E. Carr Everbach (Swarthmore College, Swarthmore, PA 19081, ceverba1@swarthmore.edu), Irina N. Chernysh, and John W. Weisel (Univ. of Penn School of Med, Philadelphia, PA 19104)

Blood clots made of human purified fibrin (white clots) were insonified with $1 \mathrm{MHz}$ pulsed ultrasound during observation by fluorescence confocal microscopy. A deconvolution microscope allowed extremely thin sheets $(0.2 \mu \mathrm{m})$ of fibrin to be viewed at a resolution of $0.2 \mu \mathrm{m}$ per pixel, and the clot microstructure visualized. Acoustic pressure amplitudes from 0.1 to $0.8 \mathrm{MPa}$ (peak-to-peak) were inferred using the image blur of 0.6$\mu$ m-diameter polystyrene spheres coated with FITC fluorecent label present in the clots. Acoustic pulse widths of $1 \mathrm{~ms}$ and pulse repetition frequencies of $125 \mathrm{~Hz}$ reduced clot heating to less than $3^{\circ} \mathrm{C}$ during each 30-minute exposure. Still $100 \mu \mathrm{m}$ by $100 \mu \mathrm{m}$ images were recorded every 10 seconds during pauses in insonificaiton, to produce time-lapse movies that are compared with movies made during sham ultrasound exposures.

\section{1:05}

2aBB10. Acousto-mechanical and thermal properties of clotted blood. Volodymyr M. Nahirnyak (Dept. of Phys., Univ. of Cincinnati, Cincinnati, OH 45221-0511), S. Wang Yoon, and Christy K. Holland (Univ. of Cincinnati, Cincinnati, OH 45267-0586)

The efficacy of ultrasound-assisted thrombolysis as an adjunct treatment of ischemic stroke is being widely investigated. In order to determine the role of ultrasound hyperthermia in the process of blood clot disruption, the thermal and acousto-mechanical properties of clotted blood were measured in vitro. Whole blood clots were prepared from either fresh porcine or human blood by aliquoting 1.5 or $2.0 \mathrm{ml}$ into $10 \mathrm{ml}$ glass tubes (BD Vacutainer ${ }^{\mathrm{TM}}$, Franklin Lakes, NJ), immersing the tubes in a $37^{\circ} \mathrm{C}$ water bath for three hours and storing the clots at $5^{\circ} \mathrm{C}$ for at least three days prior to assessment of the properties, which ensured complete clot retraction. Direct calorimetric measurements using calibrated $E$-type thermocouples (Omega Engineering, Inc., Stanford, CT) were performed to determine the heat capacity and thermal conductivity of the human and porcine thrombi against a standard fluid, saline [0.9\%]. The amplitude coefficient of attenuation of the clots was determined from $120 \mathrm{kHz}$ to 3.5 $\mathrm{MHz}$ with a calibrated hydrophone (TC4038, RESON, Inc., Goleta, CA) in a $20 \pm 2{ }^{\circ} \mathrm{C}$ water bath using the substitution method. The experimentally measured values of heat capacity, density, and thermal conductivity of porcine clotted blood are $3.23 \pm 0.46 \mathrm{~J} / \mathrm{g} \cdot \mathrm{K}, 1.058 \pm 0.014 \mathrm{~g} / \mathrm{cm}^{3}$, and $0.52 \pm 0.14 \mathrm{~W} / \mathrm{m} \cdot \mathrm{K}$. The attenuation coefficient ranged from 0.10 to 0.30 Nepers/cm over $120 \mathrm{kHz}$ to $3.5 \mathrm{MHz}$. Measurements of the acousto- mechanical and thermal properties of clotted blood can be helpful in theoretical modeling of ultrasound hyperthermia in ultrasound-assisted thrombolysis.

\section{1:20}

2aBB11. Measurements of the effect of haemolysis on speed of sound and attenuation through suspensions of red blood cells at $15 \mathrm{MHz}$. Nicola Z. Hobbs and Constantin-C. Coussios (Dept. of Eng. Sci., Univ. of Oxford, Parks Rd., Oxford OX1 3PJ, U.K.)

When blood flows through an artificial environment for prolonged periods of time, the red blood cells are gradually destroyed due to the high shear stresses to which they are exposed. The ability to measure the degree of haemolysis accurately and in real time is becoming increasingly important, especially in the context of organ preservation and of the implantation of heart-assist devices as a long-term method for treating heart failure. A recent study indicated that the backscattering coefficient (BSC) of red cell suspensions containing damaged red blood cells is significantly higher than the BSC of suspensions containing exclusively healthy cells. Using a specially designed acoustic chamber, suspensions containing different proportions of healthy and damaged cells were exposed to $15-\mathrm{MHz}$ ultrasound. A thin tungsten wire immersed in each suspension was used to quantify the speed of sound and attenuation through increasingly haemolysed suspensions. It is intended to utilize a combination of measurements of speed of sound, attenuation and backscattering in order to solve the inverse problem and to develop an on-line, non-invasive device for quantifying the degree of haemolysis in artificial circuits.

\section{$11: 35$}

2aBB12. Lytic efficacy of apoli protein E2 (ApoE2) and recombinant tissue plasminogen activator (rt-PA) treatment with $120 \mathrm{kHz}$ ultrasound in an in-vitro human clot model. Jason M. Meunier, Jason Y. Cheng (Dept. of Emergency Medicine, Univ. of Cincinnati, 231 Albert Sabin Way, Cincinnati, OH 45267, meuniejn@uc.edu), Joseph F. Clark (Univ. of Cincinnati Medical Ctr., Cincinnati, OH 45267), and George J. Shaw (Univ. of Cincinnati, Cincinnati, OH 45267)

Currently, the only FDA approved therapy for acute ischemic stroke is recombinant tissue plasminogen activator (rt-PA). However rt-PA has substantial side effects such as hemorrhage. This has led to interest in other potential therapies. For example, ultrasound (US) increases the lytic efficacy of rt-PA. Also, apolipoprotein E2 (ApoE2) increases rt-PA activity. This suggests combining US, ApoE2 and rt-PA to improve thrombolysis, but the efficacy is not known. Here, the lytic efficacy of apoE2, rt-PA and $120 \mathrm{kHz}$ US is measured in a human clot model. Whole blood was obtained from volunteers, after local institutional approval. Clots were formed in $1.7 \mathrm{~mm}$ micropipettes, and placed in a water tank that allowed microscopic video imaging during US and thrombolytic exposure. Clots 
were treated with rt-PA $([\mathrm{rt}-\mathrm{PA}]=3.15 \mu \mathrm{g} / \mathrm{ml})$, rt-PA and apoE2 $([$ apoE2 $]=9.8 \mu \mathrm{g} / \mathrm{ml})$, or rt-PA, apoE2 and $120 \mathrm{kHz}$ US $(0.35 \mathrm{MPa}$, $\mathrm{PRF}=1667 \mathrm{~Hz}, 80 \%$ duty cycle) for $15 \mathrm{~min}$ at $37^{\circ} \mathrm{C}$ in human plasma. Clot lysis was visually recorded and the lysis depth (LD) determined from these data using an image analysis algorithm. LD was linear with time for all treatments $\left(R^{2} \geqslant 0.81\right)$, allowing the determination of a lytic rate (LR).
LR was found to be $0.35 \pm 0.03,1.55 \pm 0.11$, and $0.75 \pm 0.04 \mu \mathrm{m} / \mathrm{min}$ for the rt-PA, rt-PA and apoE2, and US treated groups respectively. The thrombolytic efficacy of rt-PA is enhanced by ApoE2. The interaction of $120 \mathrm{kHz}$ with apoE2 and rt-PA showed a reduced lytic efficacy compared with rt-PA and apoE2 treatment alone. It is possible that US interferes with the ApoE2-mediated activation of rt-PA.

TUESDAY MORNING, 17 MAY 2005

PLAZA B, 10:00 A.M. TO 12:00 NOON

\title{
Session 2aED
}

\section{Education in Acoustics: Hands-On Experiments for High School Students}

\author{
Uwe J. Hansen, Chair \\ Physics Dept., Indiana Univ., Terre Haute, IN 47809 10:00
}

\begin{abstract}
Sudents and senior scientists will staff approximately 20 experiment stations for local high school students who will perform the experiments. These students will thus be exposed to a variety of acoustics principles and get some practical hands-on experience with a number of research tools. Regular ASA meeting participants are welcome to the session, as long as their participation does not interfere with student hands-on activities.
\end{abstract}

TUESDAY MORNING, 17 MAY 2005

BALMORAL, 8:30 A.M. TO 12:05 P.M.

\section{Session 2aMU}

\section{Musical Acoustics: Virtual Musical Instruments}

\author{
Julius O. Smith, Cochair \\ 4360 Miller Ave., Palo Alto, CA 94306-4410 \\ Tamara Smyth, Cochair \\ Simon Fraser Univ., School of Computer Science, Surrey, BC V3T 5X3, Canada \\ Invited Papers
}

8:30

2aMU1. A physical model and experimental testbed for real-time simulation of flute-like instruments. Patricio de la Cuadra (Ctr. for computer research in music and acoustic, CCRMA, Stanford Univ., CA 94305-8180), Benoit Fabre (Univ. Paris 6, 75252 Paris Cedex 05, France), Jonathan S. Abel (Universal Audio, Inc., Santa Cruz, CA 95060), and Julius O. Smith III (Stanford Univ., CA 94305-8180)

In the last decades, a wealth of experimental data has been gathered concerning sound production in flute-like instruments. These data have driven the development and fine tuning of analytical models for the physical processes involved. Here, a number of these results are integrated into a real-time physical model of a flute-like instrument. The model proposed is one-dimensional and driven by a single input pressure. The system was designed in the spirit of a laboratory testbed, where geometric and performance parameters can be controlled in real time, and in which important physical quantities can be viewed in real time, either in the time domain or frequency domain. In this way, the user may observe and validate the behavior of variables that would otherwise be hidden in the feedback loop. Additionally, the system was designed in a modular fashion, so as to allow independent substitution and adjustment of any of the model blocks. So as to allow real-time implementation and parameter control, a novel time-varying jet filter has been developed which not only closely matches measured jet oscillation amplitudes as a function of jet geometry and frequency, but may be rapidly slewed in response to quickly changing performance parameters. 
2aMU2. A unified digital waveguide (infra)structure for synthesizing wind instrument sounds. Gary Scavone (Music Technol., Faculty of Music, McGill Univ., 555 Sherbrooke St. West, Montreal, QC, Canada H3A 1E3)

Virtual acoustic models provide researchers a means for testing and verifying their scientific understanding of physical systems. As well, they allow explorations in a world free from physical constraints. A digital waveguide structure is presented which implements a generalized air column, or resonator, model for wind instrument sound synthesis. This system has a physical inspiration and interpretation, a "blowed string," but is an otherwise impossible reality. The structure produces harmonic resonant modes at integer multiples of a fundamental, with controls to vary harmonic content. It is useful for simulating both cylindrical and conical air columns of such instruments as clarinets, saxophones, flutes, oboes, and trumpets. Further, this structure robustly interconnects with several different non-linear "reed" models to produce a rich variety of wind instrument sounds. The result is a highly efficient computational algorithm that can be used to perform real-time sound synthesis on consumer-grade computers. [Work supported by the Canadian Foundation for Innovation.]

9:20

2aMU3. Complete geometric computer simulation of a classical guitar. Rolf Bader (Inst. of Musicology, Neue Rabenstr. 13, 20354 Hamburg, Germany)

The aim of formulating a complete model of a classical guitar body as a transient-time geometry is to get detailed insight into the vibrating and coupling behavior of the time-dependent guitar system. Here, especially the evolution of the guitars initial transient can be looked at with great detail and the produced sounds from this computer implementation can be listened to. Therefore, a stand-alone software was developed to build, calculate, and visualize the guitar. The model splits the guitar body into top plate, back plate, ribs, neck, inclosed air, and strings and couples these parts together including the coupling of bending waves and in-plane waves of these plates to serve for a better understanding of the coupling between the guitar parts and between these two kinds of waves. The resulting waveforms are integrated over the geometry and the resulting sounds show up the different roles and contributions of the different guitar body parts to the guitar sound. Here cooperation with guitar makers is established, as changes on the guitars geometry on the resulting sound can be considered as computer simulation and promising new sound qualities can then be used again in real instrument production.

\section{9:45}

2aMU4. An allpass filter design method with application to piano string synthesis. Jonathan S. Abel (Universal Audio, Inc., Santa Cruz, CA 95060), Julius O. Smith III (Stanford Univ., Stanford, CA 94305), and Julien Bensa (Universite Pierre et Marie Curie, Paris, France)

A nonparametric allpass filter design method for matching a desired group delay as a function of frequency is presented. The technique is useful in physical modeling synthesis of musical instruments exhibiting dispersive wave propagation in which different frequency bands travel at different speeds. While current group delay filter design methods suffer from numerical difficulties except at low filter orders, the technique presented here is numerically robust, producing an allpass filter in cascaded biquad form, and with the filter poles following a smooth loop within the unit circle. The technique was inspired by the observation that a pole-zero pair arranged in allpass form has $2 \pi$ total group delay when integrated around the unit circle, regardless of the pole location. To match a given group delay characteristic, the method divides the frequency axis into sections containing $2 \pi$ total group delay, and assigns a pole-zero allpass pair to each. In this way, the method incorporates an order selection technique, and by adding a pure delay to the desired group delay, allows the trading of increased filter order for improved fit to the frequency-dependent group delay. Results are presented for modeling the group delay of a stiff piano string under several computational constraints.

$$
\text { 10:10-10:20 Break }
$$

2aMU5. Music and science meet at the micro level: Time-frequency methods and granular synthesis. Barry Truax (School of Commun., Simon Fraser Univ., Burnaby, BC, Canada V5A 1S6)

Musical research over the last century has become increasingly entwined with the areas of acoustics, psychoacoustics, and electroacoustics. One of the most striking results has been to push the frontiers of models of sound and music to the micro level, what is generally termed microsound. At this level, concepts of frequency and time are conjoined by a quantum relationship, with an uncertainty principle relating them that is precisely analogous to the more famous uncertainty principle of quantum physics. A class of methods of sound synthesis and signal processing known as time-frequency models have their basis at this quantum level such that changes in a signal's time domain result in spectral alterations and vice versa. One such method, granular synthesis and the granulation of sampled sound, produces results by the generation of high densities of acoustical quanta called grains. Such a radical shift has profound implications for not only our models of sound design, but also for the compositional methods that emerge as well as the role of the composer in guiding complex processes. The paper will argue that these models are examples of a class of complex systems exhibiting emergent form that create a new form of virtual music instrument. 

Stanford, CA 94305)

Variable diffusion has been incorporated into many room acoustics models, which allows for the representation of materials with different scattering properties. By simulating the scattering of sound waves off surfaces in an acoustical enclosure, a more realistic sound field is produced. Due to constraints on the algorithm architecture and a preference for late reverberation which approaches decaying gaussian noise, it is less common for diffusion to be featured as an adjustable parameter in artificial reverberators. However, parameterized diffusion can be a tool for tailoring the character and density of the reverberant tail, enabling the generation of a greater variety of reverbs. This paper will present methods for implementing and controlling diffusion in artificial reverberation models based on digital waveguides. Sound examples will be given during the presentation.

11:10

2aMU7. Acoustics of the Intonarumori. Stefania Serafin (Medialogy, Aalborg Univ. Copenhagen, Lautrupvang 15, 2750 Ballerup, Denmark)

The Intonarumori were a family of musical instruments invented by the Italian futurist composer and painter Luigi Russolo. Each Intonarumori was made of a wooden parallelepiped sound box, inside which a wheel of different sizes and materials was setting into vibration a catgut or metal string. The pitch of the string was varied by using a lever, while the speed of the wheel was controlled by the performer using a crank. At one end of the string there was a drumhead that transmitted vibrations to the speaker. Unfortunately, all the original Intonarumori were destroyed after a fire during World War II. Since then, researchers have tried to understand the sound production mechanism of such instruments, especially by consulting the patents compiled by Russolo or by reading his book "The art of noise." In this paper we describe the acoustics of the Intonarumori. Based on such description, we propose physical models that simulate such instruments. The intonarumori's string is modeled using a one dimensional waveguide, which is excited either by an impact or a friction model. The body of the instrument is modeled using a 3-D rectangular mesh, while the horn is considered as an omnidirectional radiator.

\section{Contributed Papers}

\section{1:35}

2aMU8. Guidophone: A handheld virtual music instrument combining vocal tract geometry and hand gestures. Ryan Cassidy, Rodrigo Segnini, and Yi-Wen Liu (Ctr. for Comput. Res. in Music and Acoust., Stanford Univ., Stanford, CA 94305)

Vocal sounds provide an intuitive and appealing basis for virtual instruments. The former aspect allows most people to engage in a musical activity simply by repetition of a heard sound. Physical models in speech synthesis attempt, among other objectives, to approximate the geometry of the vocal tract required to produce specific sounds. Cook has developed one such model [Perry Cook, Identification of Control Parameters in an Articulatory Vocal Tract Model with Applications to the Synthesis of Singing, Stanford University, 1990], where the vocal tract is divided into tube sections that govern the transmission and reflection of acoustic energy at the junctions between sections. Tube section radii provide the model parameters. This paper explores the feasibility of using hand gestures to control those parameters. To that end, the problem of mapping hand gestures to the parameters of Cook's vocal synthesis model, with attention paid to transitions between successive phonemes, is explored. The physical basis of the model is reviewed. Next the relationship between the geometries implied by Cook's tract model and those determined by experiment is investigated. A means for mapping a number of parameters less than the degrees of freedom inherent in the model is presented. Finally, the details of a virtual instrument, controlled by a mechanical device recently developed for the work, are presented.

\section{1:50}

2aMU9. SCUBA: The Self-Contained Unified Bass Augmenter. Juan Pablo Cceres, Gautham J. Mysore, and Jeffrey Trevio (Ctr. for Comput. Res. in Music and Acoust., Stanford Univ., The Knoll, 660 Lomita, Stanford, CA 94305)

The Self-Contained Unified Bass Augmenter (SCUBA) is a new augmentative OSC (Open Sound Control) controller for the tuba. SCUBA allows new expressive possibilities by adding onboard continuous and discrete sensors to provide user-controlled parametric data for the processing of the instruments natural sound in Pd. SCUBA endows acoustic instruments with parametric control of virtual instruments and provides a means of integrating the output of a virtual instrument with the natural sound of the tuba. The user sends controller data from the augmented tuba interface to the virtual instrument via FSRs (Force-Sensitive Resistors) and buttons mounted on the existing instrument interface. An AVRMini microcontroller converts raw sensor data to OSC (Open Sound Control) messages, which are mapped in Pd to control virtual instrument parameters. Virtual instrument output is integrated into the solo instrument interface via satellite speakers mounted in the bell; this allows for mixing of the instruments natural sound with that of the virtual instrument to create the impression of a single instrument. This integration is the goal of the SCUBA project: by providing a flexible but unified control interface and acoustic output, traditional acoustic instrument interfaces can be augmented and paired with virtual musical instruments. [We would like to thank Michael Gurevich, Max Matthews, Bill Verplank, Pascal Stang, and classmates from Music 250 (Fall 04) for assistance in the realization of this project.] 


\title{
Session 2aNS
}

\section{Noise and Physical Acoustics: Urban Sound Propagation}

\author{
Jian Kang, Cochair \\ Univ. of Sheffield, School of Architecture, Western Bank, Sheffield S10 2TN, United Kingdom
}

Ning Xiang, Cochair

Rensselaer Polytechnic Inst., Architecture, 110 8th St., Troy, NY 12180

Chair's Introduction-8:00

Invited Papers

2aNS1. Experimental study of sound propagation in a street. Judicaël Picaut (Section acoustique routière et urbaine, LCPC, route de Bouaye, BP 4129, 44341 Bouguenais Cedex, France)

This paper presents an experimental study of the sound propagation in a street, performed during July 2002, in order to measure impulse responses at numerous locations in a street canyon, and, for several positions of the sound source. A specific attention has been paid on the processing of the experimental data, in order to obtain accurate values of the reverberation time and the steady-state sound pressure level, including also the compensation of the atmospheric attenuation. In total, 11592 useful data have been collected during this experiment, and are available by contacting the author. Reverberation times and sound levels, in the narrow street, are then analyzed and compared, for the 4 source positions in the street. This experimental study gives interesting results on the behavior of the sound field in the street.

8:30

2aNS2. A scale model study of parallel urban street canyons. Maarten Hornikx, Jens Forssen, and Wolfgang Kropp (Dept. of Appl. Acoust., Chalmers Univ. of Technol., S-412 96 Göteborg, Sweden)

The access to quiet areas in cities is of increasing importance. Recently, the equivalent sources method for a two dimensional situation of parallel urban street canyons has been developed. One canyon represents a busy road, whereas the other is one without traffic; the quiet side. With the model, the transfer function between the two canyons can be calculated, as well as the influence of diffusion, absorption, and atmospheric turbulence on the transfer function. A scale model study of two parallel canyons has now been executed. A scale of 1:40 has been chosen and the maximum length sequence technique has been applied using the MLSSA system. Results of the scale model study have been compared to calculations with the equivalent sources method. The difference between a two-dimensional and a three-dimensional quiet side, between a coherent and an incoherent line source and the influence of absorption and diffusion has been investigated. The scale model study also gives insight in the evolution of the sound field in the time domain. [Work supported by the Swedish Foundation for Strategic Environmental Research (MISTRA).]

\section{8:55}

2aNS3. The effect on sound propagation in streets of the distribution of acoustic scatterers on facades. David J. Oldham and Mostafa R. Ismail (School of Architecture, Univ. of Liverpool, Liverpool, L69 3BX, UK, djoldham@liv.ac.uk)

Until recently street noise propagation models assumed that faade reflections were either totally specular or totally diffuse. However, the characteristics of building facades are such that some energy will be reflected specularly and some diffusely. Many current models, therefore, use a scattering coefficient to characterize the diffuse reflection characteristics and the assumption is made that a fraction of the sound energy falling on any point on the facade will be scattered to the same degree. From observation it can be seen that facades are not homogeneous but consist of surfaces which are planar in nature but feature discontinuities such as windows, doors, ledges, etc. which will tend to act as distributed scattering centers. The effect of distributions of scattering centers on the propagation of sound in streets is examined using a combined image source and randomly scattered sound rays. Computer generation of street models using an object based approach enables the study of many different configurations using RAYNOISE software. Results are compared with those obtained assuming a homogeneous distribution of facade scattering using both RAYNOISE and a technique proposed by the authors. The results of a scale model experiment to investigate the effect of distributed scatters are presented.

2aNS4. Recent advances in modeling the propagation noise in high-rise cities. Kai Ming Li (Dept. Mech., The Hong Kong Polytechnic Univ., Hung Hom, Hong Kong)

In the past few decades, we have witnessed a rapid growth in mechanized transport and transportation systems. We live in a transport-dominated society which has led to a marked improvement in dispersal of land use and to the increased opportunity for the separate development of residential, commercial, and industrial areas. In dense and high-rise cities, various modes of land transpor- 
tation are the primary source of noise. The problem of transportation noise is not confined by political or social frontiers. It affects the rich who may live in a quiet residential area but who must make full use of transport to maintain their affluent existence, as well as the less fortunate who must live close to a highway, a major road, or an elevated railway line. A systematic development of the capability for accurate predictions of the propagation of land transportation noise in dense high-rise cities is highly desirable. This paper summarizes the current models for predicting sound fields in urban environments and gives an overview of the recent advances of various numerical models to predict the sound field in urban environments. [Work supported by the Research Grants Council of the Hong Kong SAR Government and the Hong Kong Polytechnic University.]

\section{9:45-10:00 Break}

\section{0:00}

2aNS5. Acoustic pulse propagation in an urban environment. Donald G. Albert (USA ERDC-CRREL, 72 Lyme Rd., Hanover, NH 03755-1290) and Lanbo Liu (USA ERDC-CRREL, Hanover, NH 03755-1290)

Experimental measurements conducted in a full-scale artificial village show that complex signatures are formed by multiple reflections and diffractions from buildings along the propagation path. A two-dimensional finite difference time domain (FDTD) simulation running on a personal computer allows this wave interaction to be studied in detail. Time reversal processing to locate a sound source in an urban area is investigated using this simulation method. The results demonstrate that as few as three non-line-ofsight sensors are sufficient to determine the source location, and that errors on the order of a meter in the building or sensor locations still allow the correct source location to be determined. [Work supported by U.S. Army.]

\section{0:25}

2aNS6. Sound field simulation and acoustic animation in urban squares. Jian Kang and Yan Meng (School of Architecture, Univ. of Sheffield, Western Bank, Sheffield S10 2TN, UK, j.kang@sheffield.ac.uk)

Urban squares are important components of cities, and the acoustic environment is important for their usability. While models and formulae for predicting the sound field in urban squares are important for their soundscape design and improvement, acoustic animation tools would be of great importance for designers as well as for public participation process, given that below a certain sound level, the soundscape evaluation depends mainly on the type of sounds rather than the loudness. This paper first briefly introduces acoustic simulation models developed for urban squares, as well as empirical formulae derived from a series of simulation. It then presents an acoustic animation tool currently being developed. In urban squares there are multiple dynamic sound sources, so that the computation time becomes a main concern. Nevertheless, the requirements for acoustic animation in urban squares are relatively low compared to auditoria. As a result, it is important to simplify the simulation process and algorithms. Based on a series of subjective tests in a virtual reality environment with various simulation parameters, a fast simulation method with acceptable accuracy has been explored. [Work supported by the European Commission.]

\section{0:50}

2aNS7. Modeling the characteristics of wheel/rail rolling noise. Wai Keung Lui, Kai Ming Li (Dept. of Mech. Eng., The Hong Kong Polytechnic Univ., Hung Hom, Hong Kong), and Glenn H. Frommer (MTR Corp. Ltd., Kowloon Bay, Hong Kong)

To study the sound radiation characteristics of a passing train, four sets of noise measurements for different train operational conditions have been conducted at three different sites, including ballast tracks at grade and railway on a concrete viaduct. The time histories computed by the horizontal radiation models were compared with the measured noise profiles. The measured sound exposure levels are used to deduce the vertical directivity pattern for different railway systems. It is found that the vertical directivity of different railway systems shows a rather similar pattern. The vertical directivity of train noise is shown to increase up to about $30 \times$ before reducing to a minimum at $90 \times$. A multipole expansion model is proposed to account for the vertical radiation directivity of the train noise. An empirical formula, which has been derived, compares well with the experimental data. The empirical model is found to be applicable to different train/rail systems at train speeds ranging up to $120 \mathrm{~km} / \mathrm{h}$ in this study. [Work supported by MTR Corporation Ltd., Innovation Technology Commission of the HKSAR Government and The Hong Kong Polytechnic University.]

\section{Contributed Papers}

\section{1:15}

2aNS8. Microscopic traffic modelling in urban noise assessment. Bert De Coensel, Dick Botteldooren, Tom De Muer (Acoust. Group, Dept. of Information Technol., Ghent Univ., St. Pietersnieuwstraat 41, B-9000 Ghent, Belgium), Bert Peeters, and Gijsjan van Blokland $(\mathrm{M}+\mathrm{P}$ Raadgevende Ingenieurs, NL-5260 CB Vught, The Netherlands)

The temporal structure of the urban soundscape can be rather complex, due to the presence of many screening and reflecting surfaces and many different sound sources, of which traffic noise is the most dominant. From the point of view of soundscape research, the background level as well as the time structure of noise peaks are important. However, these indicators cannot be estimated easily by current noise prediction models, based on static traffic flows. Therefore, a dynamic traffic noise model was used, based on a microscopic traffic simulation. This way, individual vehicles can be traced, each having an associated set of noise sources that can depend on vehicle properties such as speed and acceleration, as well as on road properties such as the surface type. The model further consists of an ISO 9613 based propagation component, which can account for multiple reflections and diffractions. Maps of statistical noise levels, but also of more complicated measures reflecting the time structure of the soundscape, can be produced. This way, the soundscape contribution of single vehicles can be traced, as well as the influence of more general vehicle properties, such as the contribution of vehicle acceleration noise to the soundscape at junctions. 
2aNS9. Increased damping in irregular resonators. Bernard Sapoval (Laboratoire de Physique de la Matiere Condensee, Ecole Polytechnique, 91128 Palaiseau, France), Mark Asch (Universite de Picardie Jules Verne, 80039 Amiens, France), Simon Felix, and Marcel Filoche (Ecole Polytechnique, 91128 Palaiseau, France)

The relation between shape and damping of shallow acoustical cavities has been studied numerically in the case where the dissipation occurs only on the cavity walls. It is first found that whatever the type of geometrical localization mechanism is what is called weak localization. The more irregular, the smaller the quality factors are found. However this effect is very different for the non-localized and the localized modes. For nonlocalized modes the damping increases roughly proportionally to the cavity surface. The localized modes are even more damped. These results generalize the results already obtained both numerically and experimentally on prefractal acoustical cavities. [B. Sapoval, O. Haeberle, and S. Russ, J. Acoust. Soc. Am. 102, $2014-2019$ (1997); B. Hebert, B. Sapoval, and S. Russ, ibid. 105, 1567-1576 (1999)].

TUESDAY MORNING, 17 MAY 2005

REGENCY B, 7:55 A.M. TO 12:00 NOON

\begin{abstract}
Session 2aPA
Physical Acoustics, Engineering Acoustics and Noise: Infrasound: A New Frontier in Monitoring the Earth I

Wayne N. Edwards, Cochair

Dept. of Earth Sciences, Univ. of Western Ontario, 1151 Richmond St., London, ON N6A 5B7, Canada

David E. Norris, Cochair

BBN Technologies, 1300 North 17th St., Arlington, VA 22209

Chair's Introduction-7:55

Invited Papers

8:00

2aPA1. Infrasound associated with the 2004 Sumatra megathrust earthquake and tsunami. Milton Garces, Pierre Caron, and Claus Hetzer (Infrasound Lab., Univ. of Hawaii, Manoa, 73-4460 Queen Kaahumanu Hwy., \#119, Kailua-Kona, HI 96740-2638)

Multiple infrasound arrays in the Pacific and Indian Oceans that are part of the International Monitoring System (IMS) observed three distinct waveform signatures associated with the December 26, 2004 Sumatra earthquake and tsunami. Infrasound station in Palau, Diego Garcia, Madagascar, and Kenya observed (1) seismic arrivals ( $P, S$ and surface) from the earthquake, (2) $T$-phases, propagated along SOFAR channel in the ocean, and coupled back to the ground, and (3) infrasonic arrivals associated with either the tsunami generation mechanism or the motion of the ground above sea level. All signals were recorded by the pressure sensors in the arrays. The seismic and $T$-phase recordings are due to the sensitivity of the MB2000 microbarometers to ground vibration, whereas the infrasound arrivals correspond to dispersed acoustic waves propagated through atmospheric waveguides. It appears that the arrival of the tsunami, as well as oceanic infragravity waves following the tsunami, were not observed by the infrasound stations. We show the prominent features of the arrivals, present source location estimates, discuss the absence of a signal associated with the tsunami arrival, and consider the potential for using infrasound as a discriminant for tsunami genesis.
\end{abstract}

8:20

2aPA2. Acoustic surveillance for hazardous eruptions (ASHE). David McCormack (Geological Survey of Canada, 7 Observatory Crescent, Ottawa, ON, Canada K1A 0Y3, cormack@seismo.nrcan.gc.ca), Henry Bass (Univ. of Mississippi, University, MS 38677), Milton Garces (Univ. of Hawaii, Manoa, Kailua-Kona, HI 96740-2638), and Hugo Yepes (Escuela Politcnica Nacional, Casilla 17-01-2759, Quito, Ecuador)

The potential of using infrasound to rapidly identify explosive volcanic eruptions has been discussed in the environmental acoustics and aviation safety communities for some time. The ability of sounds in the $0.01-10 \mathrm{~Hz}$ range to propagate for long distances with little attenuation suggests broad-area regional monitoring with a modest number of observing sites is possible. The ASHE experiment tests both the practical utility of infrasound as a regional-scale volcanic eruption detection tool, and the feasibility of using such an infrasound system to provide timely operational alerts to aviation through Volcanic Ash Advisory Centres (VAACs). Several infrasound arrays are deployed in a volcanic region, sending data in real time to a central detector, and onward to participating VAACs for comparison with existing warning systems. The ASHE experiment will determine if infrasound can complement both seismic and satellite observations to improve monitoring of volcanic hazard. Continuous acoustic surveillance can reduce the ambiguity between eruptive and purely seismic activity in an active volcano and provide additional estimates for the onset time of an eruption. The onset time estimates can be used as triggers for ash transport models. 
2aPA3. Estimation of bolide energy using observed infrasound signals. Wayne N. Edwards (Dept. of Earth Sci., Univ. of Western Ontario, London, ON, Canada N6A 5B7), Peter G. Brown (Univ. of Western Ontario, London, ON, Canada N6A 3K7), and Douglas O. ReVelle (Los Alamos Natl. Lab., Los Alamos, New Mexico, 87545)

The acoustic amplitude-yield relationships including formal errors for a population of large and well-observed (greater than 0.05 kton) bolide events have been investigated. Using various signal measurements as a function of range, these data have been calibrated against optical yield estimates from satellite measurements. A correction for the presence of stratospheric winds has also been applied to the observations and is found to greatly improve the relationship correlation, remaining scatter may be due to variations amongst the fireball population such as differing burst altitudes, greater or lesser amounts of fragmentation or the variability in the magnitude of stratospheric winds, which can during certain times of the year be comparable to or exceed the strength of the winds themselves. Comparison to point source, ground-level nuclear and high explosive airwave data with similar yields shows that observed bolide infrasound is consistently lower in amplitude. This downward shift relative to nuclear and HE data is interpreted as due in part to increased weak nonlinearity during signal propagation from higher altitudes. This is a likely explanation, since mean estimates of the altitude of maximum energy deposition along the bolide trajectory was found to be between $20-30 \mathrm{~km}$ altitude for this fireball population.

9:00

2aPA4. Numerical acoustic wave propagation in the atmosphere. Lars Ceranna (BGR, B3.11, Hannover, Germany) and Alexis Le Pichon (CEA/DASE, Bruyeres-le-Chatel, France)

A method is presented which simulates the acoustic wave-field in the atmosphere using a Chebyshev pseudo spectral approach. The aero-acoustic equation of motion is solved in spherical coordinates. The computational time is significantly reduced by applying a rotationally symmetric approximation. Since the complete wave-field is calculated for the two-dimensional model the solution provides pictures of the pressure propagation from the source to the receiver. These images make it possible to study two effects in detail: How the sound and wind profiles affect the synthetic barograms for a fixed source, and in which way the source in space and time is reflected in the synthetic recordings for a stationary elastic model. Examples for both applications are shown where measured data are compared with synthetics. The recording at the German IMS station I26DE of the September 21, 2001, chemical explosion in Toulouse is considered as a benchmark for synthetic barograms calculated for two-dimensional profiles based on NRL-G2S and MSISE/HWM. In the second example the estimation of the source functions of super-sonic moving bodies is demonstrated. The synthetic waveforms are compared with the signals of two bolides recorded at the French IMS station I24FR at Tahiti from the December 1, 2003.

\section{9:20}

2aPA5. Contribution of infrasound monitoring for atmospheric investigations. Alexis Le Pichon (CEA/DASE, BP12, Bruyeres-le-Chatel 91680, France) and Doug Drob (Naval Res. Lab., Washington, DC 20375)

The French NDC currently receives data from a number of IMS-type infrasound stations. Continuous automatic processing of the data is being performed in the $[0.02-4] \mathrm{Hz}$ frequency band in order to detect and characterize coherent infrasonic waves. Known and quasi permanent infrasonic sources are needed to evaluate and improve upper-wind models. Microbaroms are detected throughout the year on a global scale. Their monitoring reveals clear periodic trends in the detected bearings, providing further confirmation that long-range propagation strongly depends on the atmospheric conditions, primarily on the seasonal variability of the zonal winds. Infrasounds generated by active volcanoes also offer a unique opportunity for atmospheric studies. Recent observations in Vanuatu (New-Hebrides Islands) covering one full year show a maximal peak-to-peak azimuth deviation of $\sim 15$. Infrasound observations are used as input of an inversion procedure to evaluate more precisely the vertical structure of the wind above the stratosphere in a range of altitude unaccessible to ground based or satellite measurements. With the increasing number of IMS stations being deployed, continuing systematic investigations into infrasonic signals from volcanoes will certainly help to advance the development of operational infrasound monitoring, and significant contributions to the understanding of atmospheric propagation could be expected.

\section{9:40}

2aPA6. Detecting and characterizing infrasound signals with optical fiber infrasound sensors. Kristoffer Walker, Mark Zumberge, Jonathan Berger, Michael Hedlin (Inst. of Geophys. and Planetary Phys., Scripps Inst. of Oceanogr., Univ. of California, San Diego, La Jolla, CA 92093-0225), and Stephen Arrowsmith (Univ. of California, San Diego, La Jolla, CA 92093-0225)

Optical Fiber Infrasound Sensors (OFIS) are long compliant tubes wrapped with two optical fibers that interferometrically measure the differential pressure variation along the length of the tube. Because each sensor averages spatially along the length of the tube, the frequency response of the recorded pressure variation is a function of the orientation of the OFIS sensor relative to the back azimuth and incidence angle of the incoming wave. We have exploited this property to investigate the ability of various OFIS geometries to determine the back azimuth of infrasound signals. We have found that an OFIS comprised of two orthogonal 89-m-long arms having their centers separated by $63 \mathrm{~m}$ can resolve the back azimuth of most infrasound signals with a good signal-to-noise ratio. We find a good match between the back azimuths determined with our technique and those determined for the same signals recorded on the co-located pipe array I57US with the Progressive Multichannel Cross-Correlation technique. Based on these results and additional synthetic tests, we have built and are testing a larger OFIS with several arms that will be able to resolve signals from all directions and with small signal-to-noise ratios. 


\section{Contributed Papers}

\section{0:30}

2aPA7. Listening to shower meteors with infrasound. Wayne $\mathrm{N}$. Edwards (Dept. of Earth Sci., Univ. of Western Ontario, London, ON, Canada N6A 5B7), Peter G. Brown (Univ. of Western Ontario, London, ON, Canada N6A 3K7), Pavel Spurny (Astronomical Inst. of the Acad. of Sci. of the Czech Republic, Czech Republic), and Douglas O. ReVelle (Los Alamos Natl. Lab., Los Alamos, NM 87545)

Observations of the Leonid and Perseid meteor showers over the last few years in Europe using multiple camera systems, including the European Fireball Camera Network, have shown several bright meteors (abs. magnitudes -8 to -16 ) passing nearby to the IMS infrasound array in Freyung, Germany (I26DE). Subsequent checks of I26DE data show that these meteors were also detected infrasonically. This combination of optical location of the meteor in flight and microbarometer array beamforming has provided an excellent opportunity to delimit the altitudes at which these infrasound signals are being generated. UKMO temperature and wind data from the UARS satellite has been combined with MSIS and HWM models to reconstruct the acoustic velocity conditions present during observations. Using the reconstructed conditions, geometric ray tracing indicates that the source altitudes lie between approximately 80 to 105 kilometers; array back-azimuths appear to confirm this conclusion. This is extraordinary since meteoroids of this size, at these altitudes in the atmosphere, are in the transitional region from free-molecular flow to continuum flow. With these observations there is now confirmed infrasound from three separate meteor showers; the Leonids, Geminids and Perseids, meaning that meteor shower infrasound is much more common than previously thought.

\section{0:45}

2aPA8. Infrasound remote sensing of the upper atmosphere. Douglas Drob, Micheal Picone (E.O. Hulburt Ctr. for Space Res., Naval Res. Lab., 4555 Overlook Ave., Washington, DC 20375), and Robert Meier (School of Computational Sci., Fairfax, VA 22030)

We investigate the prospects for utilizing infrasound signals from geophysical sources to improve our knowledge of upper atmospheric winds and temperatures. We present results from numerical experiments designed to quantify the statistical performance of an idealized infrasonic upper atmospheric remote sensing network over a range of propagation modeling assumptions, source types, and geophysical variables. For a series of hypothetical events, infrasonic observables are calculated with a spherical 3-D ray tracer and series of atmospheric profiles spanning the range of geophysical parameters. These synthetic measurements are then inverted to estimate the original atmospheric background fields. A nonlinear least-squares estimation procedure that accounts for uncertainties in both the dependent and independent variables is used. The retrieved atmospheric profiles are represented by a set of truncated empirical orthogonal functions to incorporate a priori knowledge of atmospheric structure and reduce the number of parameters. Our numerical experiments indicate that it is indeed possible to make meaningful estimates of upper atmospheric environmental profiles with today's infrasound networks. [Work supported by the Office Naval Research.]

\section{1:00}

2aPA9. Scattering of sound and infrasound waves by internal gravity waves in the atmosphere. Vladimir E. Ostashev (NOAA/ETL 325 Broadway, Boulder, CO 80305 and Phys. Dept., New Mexico State Univ., Las Cruces, NM 88003), Igor P. Chunchuzov (Obukhov Inst. of Atmospheric Phys., Moscow, Russia), and D. Keith Wilson (U.S. Army Engineer Res. and Development Ctr., Hanover, NH 03755)

Internal gravity waves (IGW) propagating in the atmosphere produce highly anisotropic fluctuations in temperature and wind velocity that are stretched in a horizontal direction. These fluctuations can significantly affect sound propagation in a night-time boundary layer and infrasound propagation in the stratosphere. In this paper, 3-D spectra of temperature and wind velocity fluctuations due to IGW, recently derived in the literature for the limiting case of large wave numbers, are generalized to account for small wave numbers. The generalized 3-D spectra are then used for development of a theory of sound propagation through and scattering by IGW. First, the sound scattering cross-section due to IGW is calculated. It is shown that the dependence of this scattering cross-section on parameters of the problem (the scattering angle, sound frequency, etc.) is qualitatively different from that for the case of sound scattering by isotropic turbulence. Second, the mean sound field and the transverse coherence function of a plane sound wave propagating in the atmosphere with IGW are calculated and compared with those for sound propagating through isotropic turbulence. [Work supported by ARO, Grant DAAD19-01-10640, and RFBR, Grant 03-05-04001.]

\section{1:15}

2aPA10. Parabolic equation (PE) model approximations and implications for infrasound. David E. Norris (BBN Technologies, 1300 N. 17th St., Ste. 400, Arlington, VA 22209, dnorris@bbn.com)

The continuous-wave parabolic equation (PE) model is widely used in the prediction of atmospheric propagation. In this study, the effects of several PE approximations are evaluated in the context of long-range infrasonic propagation. Specifically, the focus is on quantifying: phase errors resulting from different split-step Fourier (SSF) implementations, solution stability with respect to step size, and prediction sensitivity to the choice of reference sound speed. The tradeoff between improved performance gain and increased computational loading will also be considered. The study will include comparison of PE waveform predictions with measurements from infrasonic events. These comparisons are of interest in assessing the PE modeling performance, applicability, and limitations. Waveform predictions are made by integrating the continuous-wave PE model into a Fourier-synthesis Time-domain PE (TDPE).

\section{1:30}

2aPA11. Modeling volcanic infrasound propagation using the parabolic equation. Geoffrey F. Edelmann, Douglas P. Drob, Joseph F. Lingevitch, Michael D. Collins (Naval Res. Lab., 4555 Overlook Ave. SW, Washington DC, 20375), and Alexis Le Pichon (CEA/DASE BP12, 91680 Bruyeres-le Chatel, France)

Infrasound originating from volcanic sources in the $1-4 \mathrm{~Hz}$ range is detectable at long ranges by remote monitoring stations. The effects of wind, in addition to the atmospheric sound speed, is non-negligible for the propagation of these signals. Recent developments in parabolic equation (PE) methods are applicable to infrasonic propagation where both wideangles and high-Mach number atmospheric flows are important. We apply this model to infrasonic data measured from volcanoes in the Archipelago of Vanuatu in Oceania (e.g., LePichon et al., 2005), as well as other potentially active volcanoes. The diurnal variations and seasonal variations in measured pressure amplitudes are modeled in the context of recent multi-year observations of volcanic infrasound. The significance of upper atmospheric tidal amplitudes, range dependence, and prevailing gravity waves structure in these calculations are also considered. [Work supported by ONR.]

\section{$11: 45$}

2aPA12. Use of improved propagation models to investigate novel infrasound signals. Joydeep Bhattacharyya and Robert Gibson (BBN Technologies, 1300 N. 17th St., \#400, Arlington, VA 22209, joydeep@bbn.com)

The recent availability of improved infrasound propagation modeling tools and the installation of infrasound arrays in regions of sparse cover- 
age provide new opportunities for analysis of rare signals. Analyses are presented resulting from recent natural and manmade events, and insights are discussed. Events of interest include the train explosion in Iran of February 18, 2004 and the South Asian tsunami event of December 26, 2004. Using near-real-time environmental profiles and infrasound propa- gation models, it is shown that the predicted travel times, azimuth deviation and signal duration closely match the observed values. As the dataset of such events is small, predictive modeling can be a valuable tool in developing detection strategies for such events and may ultimately lead to a decreased incidence of false alarms in global monitoring.

\title{
Session 2aPP
}

\section{Psychological and Physiological Acoustics and Speech Communication: Current Issues in Auditory Spectral Integration}

\author{
Lawrence L. Feth, Cochair \\ Ohio State Univ., Speech and Hearing Science, 1070 Carmack, Columbus, OH 43210-1372 \\ Robert A. Fox, Cochair \\ Ohio State Univ., Speech and Hearing Science, 1070 Carmack, Columbus, OH 43210-1372
}

Chair's Introduction-9:00

Invited Papers

9:05

2aPP1. Spectral integration in speech and non-speech sounds. Ewa Jacewicz (Dept. Speech and Hearing Sci., Ohio State Univ., Columbus, OH 43210-1002)

Spectral integration (or formant averaging) was proposed in vowel perception research to account for the observation that a reduction of the intensity of one of two closely spaced formants (as in /u/) produced a predictable shift in vowel quality [Delattre et al., Word 8, 195-210 (1952)]. A related observation was reported in psychoacoustics, indicating that when the components of a two-tone periodic complex differ in amplitude and frequency, its perceived pitch is shifted toward that of the more intense tone [Helmholtz, App. XIV (1875/1948)]. Subsequent research in both fields focused on the frequency interval that separates these two spectral components, in an attempt to determine the size of the bandwidth for spectral integration to occur. This talk will review the accumulated evidence for and against spectral integration within the hypothesized limit of 3.5 Bark for static and dynamic signals in speech perception and psychoacoustics. Based on similarities in the processing of speech and non-speech sounds, it is suggested that spectral integration may reflect a general property of the auditory system. A larger frequency bandwidth, possibly close to 3.5 Bark, may be utilized in integrating acoustic information, including speech, complex signals, or sound quality of a violin.

\section{9:25}

2aPP2. The dispersion-focalization theory of sound systems. Jean-Luc Schwartz, Christian Abry, Louis-Jean Boë, Nathalie Vallée (Institut de la Commun. Parlée, UMR 5009 CNRS, INPG, Université Stendhal, 46 Av. Félix Viallet, 38031 Grenoble Cedex 1, France), and Lucie Ménard (Univ. du Québec à Montréal, Montréal, Canada H3C 3P8)

The Dispersion-Focalization Theory states that sound systems in human languages are shaped by two major perceptual constraints: dispersion driving auditory contrast towards maximal or sufficient values [B. Lindblom, J. Phonetics 18, 135-152 (1990)] and focalization driving auditory spectra towards patterns with close neighboring formants. Dispersion is computed from the sum of the inverse squared inter-spectra distances in the $(F 1, F 2, F 3, F 4)$ space, using a non-linear process based on the 3.5 Bark critical distance to estimate $F 2^{\prime}$. Focalization is based on the idea that close neighboring formants produce vowel spectra with marked peaks, easier to process and memorize in the auditory system. Evidence for increased stability of focal vowels in short-term memory was provided in a discrimination experiment on adult French subjects [J. L. Schwartz and P. Escudier, Speech Comm. 8, 235-259 (1989)]. A reanalysis of infant discrimination data shows that focalization could well be the responsible for recurrent discrimination asymmetries [J. L. Schwartz et al., Speech Comm. (in press)]. Recent data about children vowel production indicate that focalization seems to be part of the perceptual templates driving speech development. The Dispersion-Focalization Theory produces valid predictions for both vowel and consonant systems, in relation with available databases of human languages inventories.

\section{9:45}

2aPP3. Exploring the role of spectral integration in the perception of vowel sounds. Randy L. Diehl (Dept. of Psych. and Ctr for Perceptual Systems, Univ. of Texas, Austin, TX 78712)

Building on the important work of Chistovich and Lublinskaya [Hearing Res. 1, 185-195 (1979)], Syrdal [Speech Commun. 4, 121-135 (1985)] proposed that vowel category boundaries tend to occur in quantal regions corresponding to a 3.5 Bark bandwidth of spectral integration. This proposal was shown to be consistent with acoustic distributions of certain American English vowels. Some initial perceptual results [K. A. Hoemeke and R. L. Diehl, J. Acoust. Soc. Am. 96, 661-674 (1994)] also tended to support the role 
of a 3.5 Bark integrator in determining major perceptual boundaries among vowel categories. However, other evidence, reviewed here, raises doubts about the generality of Syrdal's proposal. The negative evidence includes both within- and cross-language variation in the location of vowel category boundaries assumed to be governed by the 3.5 Bark integrator and the absence of elevated discriminability in putative quantal regions corresponding to the spectral limit of integration. [Work supported by NIDCD.]

10:05-10:20 Break

10:20

2aPP4. Similarities and differences in the perception of formant frequency transitions and center-of-gravity glides. Valentina Lublinskaja (Pavlov Inst. of Physiol., Russian Acad. of Sci., Makarova nab. 6, St.-Petersburg, 199034, Russia, valub@infran.ru)

In Lublinskaja (1996) the spectral "center-of-gravity" (COG) effect was observed in the identification of synthetic vowels in which the amplitude ratio of $F 2$ and $F 3$ was gradually changed in time, formant frequencies being constant. The stimuli with such virtual formant transitions (VT) were perceived as diphthong-like Russian vowels when $F 3-F 2<4.5$ Bark. Within this $F 3-F 2$ frequency separation, the stimuli with the dynamic perceptual formant $(F 2 *)$ were identified as their equivalent vowels with real $F 2$ and $F 3$ transitions (FT). But the perception of both types of transitions was not identical. Generally, the VT signals were less salient than FT. Within smaller $F 2-F 3$ separations $(F 2 *=1.68$ Bark), the VT signals were first identified with $100 \%$-accuracy as diphthong-like vowels and with larger $F 2^{*}$ the glide transitions were gradually less perceptible. With separations $F 3-F 2$ $>4.5$ Bark the VT stimuli were identified as stationary vowels, and listeners' decisions were based on terminal values of $F 2 *$. Reduced salience of VT glides relative to FM tones was also reported for non-speech signals [Dawson and Feth, ARLO 5, 95-99 (2004)]. The talk will discuss possible mechanisms for why VT signals are less perceptually prominent than FT sounds.

10:40

2aPP5. Spectral integration in the processing of dynamic place cues for stops. Robert Allen Fox and Marc Smith (Dept. Speech and Hearing Sci., Ohio State Univ., Columbus, OH 43210-1002)

Chistovich and her colleagues have proposed the spectral "center-of-gravity" (COG) hypothesis suggesting that for static signals (such as steady-state vowels) spectral integration of two or more formant peaks may occur over a range of 3.5 Bark. More recently, studies have examined dynamic COG effects by modifying the amplitude of formants in time in order to create diphthong percepts. This presentation will review data demonstrating the COG effect in the processing of dynamic cues to place of articulation in stops in both voiced [da]-[ga] and voiceless [ta]-[ka] series. By changing only the amplitudes over time of a pair of sinewaves (in [da]-[ga]) or a pair of narrow band noise signals (in [ta]-[ka]) a "virtual $F 3$ glide" can be created which can serve to signal the alveolar-velar place distinction. If these signals are played to listeners without being inserted into a base token, they are heard simply as frequency glides and not as two separate tones. Listener identification and discrimination responses to $\mathrm{CV}$ tokens with virtual and real $F 3$ frequency transitions show similar overall patterns, but virtual transitions are less salient place cues than real transitions. An auditory model incorporating the dynamic COG effect will be described.

11:00

2aPP6. Auditory spectral integration of dynamic signals. Lawrence Feth (Dept. of Speech and Hearing Sci., Ohio State Univ., Columbus, OH 43210, feth.1@osu.edu), Ashok Krishnamurthy, and Daniel Hack (Ohio State Univ., Columbus, OH 43210)

Auditory spectral integration often refers to the ability of the auditory system to sum information across a wide frequency range to improve the detectability of a supra-critical bandwidth signal in a masking experiment. However, in other paradigms, the spectral components of these broad bandwidth signals can be resolved by auditory system. These findings lead to a resolution versus integration paradox for the frequency domain similar to the resolution versus integration paradox in the time domain that led to the multiple-looks hypothesis [N. F. Viemeister and G. H. Wakefield, J. Acoust. Soc. Am., 90, 858-865 (1991)]. In speech, spectral integration refers to finding that two or more resonance peaks in a synthetic vowel can be approximated by a single peak located at the spectral center-of-gravity (COG). In 1996, Lublinskaja demonstrated that changing the COG of a two-resonance signal over time leads listeners to hear a virtual frequency transition that follows the dynamic COG. Dynamic signals may require the auditory system to take multiple looks in both time and frequency. This talk will present a series of psychoacoustic experiments using dynamic signals and a computational model that incorporates peripheral auditory processing and a modification of the PSC model.

\section{Contributed Papers}

\section{1:20}

2aPP7. Difference between diotic and dichotic presentations on audiovisual synchronization. Rie Nakamura and Kiyoaki Aikawa (School of Media Sci., Tokyo Univ. of Technol., 1404-1 Katakuracho, Hachioji, Tokyo 192-0982, Japan, aik@media.teu.ac.jp)

Significant differences were found between diotic and dichotic presentations of auditory stimuli on audiovisual synchronization. Two types of moving sphere were used for visual stimuli; (a) a sphere moved straight from left to right at a constant velocity, and (b) it moved up and right and then turned down and right at the midpoint. Auditory stimuli included (1) a continuous pure tone $(1000 \mathrm{~Hz}),(2)$ a sequence of two short tones of 30 ms $(1000 \mathrm{~Hz})$, (3) a sweep tone $(500 \mathrm{~Hz} \rightarrow 1500 \mathrm{~Hz})$, and (4) a Frequency-Modulated tone $(500 \mathrm{~Hz} \rightarrow 1500 \mathrm{~Hz} \rightarrow 500 \mathrm{~Hz})$. The duration of these stimuli was $240 \mathrm{~ms}$ each. Visual stimuli were presented on a Liquid-Crystal Display. Auditory stimuli were presented by headphones. The time difference between auditory and visual stimuli was changed between $-360 \mathrm{~ms}$ and $+360 \mathrm{~ms}$. The sound source linearly moved from left to right in case of dichotic presentation. Subjective tests, using the forced-choice of synchronized or not, clarified the significant differences between diotic and dichotic presentations when the auditory stimulus (1) or (4) was presented with the visual stimulus (b). No significance was obtained for other combinations of auditory and visual stimuli. [Work supported by NTT.] 
ditions. For auditory speech recognition, the most important frequency regions tend to be around $1500-3000 \mathrm{~Hz}$, corresponding roughly to important acoustic cues for place of articulation. The purpose of this study is to determine the most important frequency region under auditory-visual speech conditions. Frequency band-importance functions for auditory and auditory-visual conditions were obtained by having subjects identify speech tokens under conditions where the speech-to-noise ratio of different parts of the speech spectrum is independently and randomly varied on every trial. Point biserial correlations were computed for each separate spectral region and the normalized correlations are interpreted as weights indicating the importance of each region. Relations among frequencyimportance functions for auditory and auditory-visual conditions will be discussed.

TUESDAY MORNING, 17 MAY 2005

REGENCY D, 8:00 A.M. TO 12:00 NOON

\title{
Session 2aSC
}

\section{Speech Communication: Language Acquisition, Bilingual Studies and Category Learning (Poster Session)}

\author{
Murray J. Munro, Chair \\ Dept. of Linguistics, Simon Fraser Univ., 8888 Univeristy Dr., Burnaby, BC V5A 1S6, Canada
}

\section{Contributed Papers}

\begin{abstract}
All posters will be on display from 8:00 a.m. to 12:00 noon. To allow contributors an opportunity to see other posters, contributors of odd-numbered papers will be at their posters from 8:00 a.m. to 10:00 a.m. and contributors of even-numbered papers will be at their posters from 10:00 a.m. to 12:00 noon.
\end{abstract}

2aSC1. $L 1$ literacy affects $L 2$ pronunciation intake and text vocalization. Martin Walton (Dept. Langues, Université Montesquieu Bordeaux 4, Ave. Léon Duguit, 33608 PESSAC Cedex, France)

For both deaf and hearing learners, $L 1$ acquisition calls on auditive, gestural and visual modes in progressive processes over longer stages imposed in strictly anatomical and social order from the earliest prelexical phase [Jusczyk (1993), Kuhl \& Meltzoff (1996)] to ultimate literacy. By contrast, $L 2$ learning will call on accelerating procedures but with restricted input, arbitrated by $L 1$ literacy as can be traced in the English of French-speaking learners, whether observed in spontaneous speech or in text vocalization modes. An inventory of their predictable omissions, intrusions and substitutions at suprasegmental and syllabic levels, many of which they can actually hear while unable to vocalize in real-time, suggests that a photogenic segmentation of continuous speech into alphabetical units has eclipsed the indispensable earlier phonogenic module, filtering $L 2$ intake and output. This competing mode analysis hypothesizes a critical effect on $L 2$ pronunciation of $L 1$ graphemic procedures acquired usually before puberty, informing data for any Critical Period Hypothesis or amounts of $L 1$ activation influencing $L 2$ accent [Flege $(1997,1998)]$ or any psychoacoustic French deafness with regard to English stress-timing [Dupoux (1997)]. A metaphonic model [Howell \& Dean (1991)] adapted for French learners may remedially distance $L 1$ from $L 2$ vocalization procedures.

2aSC2. Lexical representation of novel $\mathbf{L 2}$ contrasts. Rachel HayesHarb (Dept of Linguist., Univ. of Utah, 255 S. Central Campus Dr., Rm. 2328, Salt Lake City, UT 84112-0492, hayes-harb@linguistics.utah.edu) and Kyoko Masuda (Georgia Inst. of Technol., Atlanta, GA 30332-0375)

There is much interest among psychologists and linguists in the influence of the native language sound system on the acquisition of second languages (Best, 1995; Flege, 1995). Most studies of second language
(L2) speech focus on how learners perceive and produce L2 sounds, but we know of only two that have considered how novel sound contrasts are encoded in learners' lexical representations of L2 words (Pallier et al., 2001; Ota et al., 2002). In this study we investigated how native speakers of English encode Japanese consonant quantity contrasts in their developing Japanese lexicons at different stages of acquisition (Japanese contrasts singleton versus geminate consonants but English does not). Monolingual English speakers, native English speakers learning Japanese for one year, and native speakers of Japanese were taught a set of Japanese nonwords containing singleton and geminate consonants. Subjects then performed memory tasks eliciting perception and production data to determine whether they encoded the Japanese consonant quantity contrast lexically. Overall accuracy in these tasks was a function of Japanese language experience, and acoustic analysis of the production data revealed non-nativelike patterns of differentiation of singleton and geminate consonants among the L2 learners of Japanese. Implications for theories of L2 speech are discussed.

2aSC3. L2 use and stimulus complexity in perceived acceent ratings. Astrid Zerla Doty, Ruth Huntley Bahr (Dept. of Commun. Sci. and Disord., Univ. of South Florida, PCD 1017, 4202 E. Fowler Ave., Tampa, FL 33620, rbahr@chuma1.cas.usf.edu), and Judith Becker Bryant (Univ. of South Florida, Tampa, FL 33620)

Listener perception of accentedness has been shown to be influenced by experience with L2 (measured by length of residence in US). However, frequency of L1 use and degree of linguistic complexity (defined by the number of non-native phonetic features targeted) may provide more insight into the role of experience in the perception of accentedness. Three groups of listeners (monolingual English and Spanish [L1] speakers divided into two groups of high and low use of English [L2]) rated the accentedness of bilingual speakers who spoke with varying degrees of accentedness. The speakers read sentences adapted from Magan (1998) to 
include linguistic aspects likely to be difficult for native Spanish speakers. Listeners performed similarly in rating speakers degree of accent. Amount of daily L1 use only influenced the ratings of the slightly accented group; the high-use bilingual group rated these speakers as more accented than the native English group, regardless of level of linguistic difficulty. These results suggest that the high-use groups lack of L2 experience made them less perceptually sensitive to certain phonetic features of English. Because speakers did not make the predicted target errors, the listener groups may have based their ratings on features not targeted in this investigation.

2aSC4. Training for learning Mandarin tones: A comparison of production and perceptual training. Xinchun Wang (Dept. of Linguist., California State Univ., Fresno, 5245 N. Backer Ave., M/S PB 92, Fresno, CA 93740)

Mandarin Chinese lexical tones pose difficulties for non-native speakers whose first languages contrast or do not contrast lexical tones. In this study, both tone language and non-tone language speaking learners of Mandarin Chinese were trained for three weeks to identify the four Mandarin lexical tones. One group took the production training with both visual and audio feedback using Kay Sona Speech II software. The target tones produced by native Mandarin speakers were played back through a pair of headphones and the pitch contours of the target tones were displayed on the computer screen on the top window to be compared with the trainees productions which appear in real time in the bottom window. Another group of participants took the perceptual training only with fourway forced choice identification tasks with immediate feedback. The same training tokens were used in both training modes. Pretest and post test data in perception and production were collected from both groups and were compared for effectiveness of training procedures.

2aSC5. Production quality of / $r /$ and /// liquids among Cantonese and Mandarin ESL learners. Donald Derrick (Dept. of Linguist., Univ. of British Columbia, 1866 Main Mall, Buchanan E270, UBC, Vancouver, BC, Canada V6T 1Z1, dderrick@interchange.ubc.ca)

Perceptual interference theories suggest L2 language learners produce phonemes based on their native language phonology (Iverson et al., 2004, Cognition). This present study investigated the impact of differing native language segmental inventories on the acquisition of the English r/l contrast. Northern Mandarin dialects exhibit coda /r/ similar to the English bunched /r/, while Cantonese exhibits no r-like liquids (Gick et al., 2003, under review). The Mandarin segmental inventory provides more of a basis for acquiring the English $\mathrm{r} / \mathrm{l}$ contrast than the Cantonese inventory. It is therefore predicted that Mandarin speakers will acquire the $\mathrm{r} / \mathrm{l}$ contrast with a lower level of experience with English than the Cantonese speakers. One Cantonese and two Mandarin ESL learners produced r/l sounds in minimally contrastive English words in simple and complex onset, coda, and intervocalic positions. The data were analyzed in two ways. Four native English listeners were asked to judge for each word whether the target consonant was /r/ or /1/. Also, ultrasound tongue images were analyzed for component /r/ and /1/ gestures. Results provided partial support for the hypothesis. Implications for theories of second language acquisition will be discussed.

2aSC6. Native and non-native perception of phonemic length contrasts in Japanese: Effects of speaking rate and presentation context. Amanda Wilson, Hiroaki Kato (ATR Human Information Sci. Labs., Kyoto 619-0288, Japan, kato@atr.jp), and Keiichi Tajima (Hosei Univ., Tokyo 102-8160, Japan)

Japanese words can be distinguished by the length of phonemes, e.g., "chizu" (map) versus "chiizu" (cheese). Perceiving these length contrasts is therefore important for learning Japanese as a second language. The present study examined native English listeners' perception of length contrasts at different speaking rates and in different contexts. Stimuli con- sisted of 20 Japanese word pairs that minimally contrasted in vowel length, and 10 synthesized nonwords. The nonwords were created by modifying the duration of the second vowel of the nonword "erete" along a continuum (from "erete" to "ereete"). Stimuli were presented with or without a carrier sentence at three rates (fast, normal, slow). Rate was either fixed or randomized trial by trial. Sixteen native English and 16 native Japanese listeners participated in a single-stimulus, two-alternative forced-choice identification task. Results suggest that native Japanese listeners' identification boundaries systematically shifted due to changes in speaking rate when the stimuli were in the context of a sentence with mixed rates of presentation. In contrast, native English listeners show a shift in the opposite direction, suggesting that they did not follow the variation in speaking rate. These results will be discussed from the viewpoint of training second-language phoneme perception. [Work supported by JSPS.]

2aSC7. Children's abilities to distinguish novel languages. Z. S. Bond and Verna Stockmal (Dept. of Linguist., Ohio Univ., Athens, OH 45701)

When adults hear spoken samples of a language which they do not know, they can often identify it and discriminate between languages even when produced by the same talkers. Children have much less experience making metalinguistic judgments. How do children respond to languages which they do not know? We have conducted three experiments examining the abilities of 4-year old and 8-year old children to discriminate between spoken samples of different languages produced by bilingual talkers. We constructed listening tests from 5-second phrases excerpted from fluent reading provided by the talkers. In the three experiments, we progressively simplified the response mode employed by the children as well as the cognitive load of the task. Even in the simplest version, only a third of the 4-year-olds could do the task while the 8-year old children performed above chance in all three experiments. The younger children tended to respond different more than same, as if their criterion for same was identify.

2aSC8. Innovative/ye/ and /we/ sequences in recent loans in Japanese. Timothy Vance and Yuka Matsugu (Univ. of Arizona, East Asian Studies, P.O. Box 210105, Tucson, AZ 85721-0105)

The GV sequences/ye/ and /we/ do not occur in Japanese except perhaps in recent loans. Katakana spellings of the relevant loans in authoritative dictionaries are inconsistent, and it is not clear whether native speakers treat them as containing the GV sequences /ye/ and /we/ or as containing the VV sequences/ie/ and /ue/. Native speakers of Japanese with minimal exposure to spoken English were recorded producing some relevant loans in response to picture prompts. The same speakers were also recorded producing some native words containing uncontroversial /ie/ and /ue/ sequences. All the productions are being analyzed acoustically to determine whether they show the expected contrast between GV and VV sequences. A VV sequence is disyllabic (and bimoraic) and should therefore have greater duration and more gradual formant movements than a monosyllabic (and monomoraic) GV sequence. Utterance-initially, a VV sequence should have a LH pitch pattern and should be preceded by a nondistinctive glottal stop, whereas a GV sequence should have a $\mathrm{H}$ pitch pattern and should have smooth onset.

2aSC9. Categorization of speech sounds by Norwegian/English bilinguals. Audny T. Dypvik and Elzbieta B. Slawinski (Psych. Dept., Univ. of Calgary, 2500 University Dr., Calgary, AB, Canada T2N 1N4, atdypvik@ucalgary.ca)

Bilinguals who learned English late in life (late bilinguals) as opposed to those who learned English early in life (early bilinguals) differ in their perception of phonemic distinctions. Age of acquisition of a second language as well as depth of immersion into English is influenced by perceptual differences of phonemic contrasts between monolinguals and bilin- 
guals, with consequences for speech production. The phonemes $/ \mathrm{v} /$ and $/ \mathrm{w} /$ are from the same category in Norwegian, rendering them perceptually indistinguishable to the native Norwegian listener. In English, /v/ and /w/ occupy two categories. Psychoacoustic testing on this phonemic distinction in the current study will compare perceptual abilities of monolingual English and bilingual Norwegian/English listeners. Preliminary data indicates that Norwegian/English bilinguals demonstrate varying perceptual abilities for this phonemic distinction. A series of speech sounds have been generated by an articulatory synthesizer, the Tube Resonance Model, along a continuum between the postures of $/ \mathrm{v} /$ and /w/. They will be presented binaurally over headphones in an anechoic chamber at a sound pressure level of $75 \mathrm{~dB}$. Differences in the perception of the categorical boundary between $/ \mathrm{v} /$ and $/ \mathrm{w} /$ among English monolinguals and Norwegian/English bilinguals will be further delineated.

2aSC10. Perception of coarticulated tones by non-native listeners. Tessa Bent (2016 Sheridan Rd., Evanston, IL 60208, t-bent@ northwestern.edu)

Mandarin lexical tones vary in their acoustic realization depending on the surrounding context. Native listeners compensate for this tonal coarticulation when identifying tones in context. This study investigated how native English listeners handle tonal coarticulation by testing native English and Mandarin listeners discrimination of the four Mandarin lexical tones in tri-syllabic sequences in which the middle tone varied while the first and last tones were held constant. Three different such frames were tested. As expected, Mandarin listeners discriminated all pairs in all contexts with a high degree of accuracy. English listeners exhibited poorer discrimination than Mandarin listeners and their discrimination accuracy showed a high degree of context dependency. In addition to assessing accuracy, reactions times to correctly discriminated different trials were entered into a multidimensional scaling analysis. For both listener groups, the arrangement of tones in perceptual space varied depending on the surrounding context suggesting that listeners attend to different acoustic attributes of the target tone depending on the surrounding tones. These results demonstrate the importance for models of cross-language speech perception of including contextual variation when characterizing the perception of non-native prosodic categories. [Work supported by NIH/ NIDCD]

2aSC11. Plasticity in speech production and perception: A study of accent change in young adults. Bronwen G. Evans and Paul Iverson (Dept. of Phonet. and Linguist., Univ. College London, Wolfson House, 4, Stephenson Way, London, NW1 2HE, UK bron@phon.ucl.ac.uk)

This study investigated plasticity in speech production and perception among university students, as individuals change their accent from regional to educated norms. Subjects were tested before beginning university, 3 months later and on completion of their first year of study. At each stage they were recorded reading a set of test words and a short passage. They also completed two perceptual tasks; they found best exemplar locations for vowels embedded in carrier sentences and identified words in noise. The results demonstrated that subjects changed their spoken accent after attending university. The changes were linked to sociolinguistic factors; subjects who were highly motivated to fit in with their university community changed their accent more. There was some evidence for a link between production and perception; between-subject differences in production and perception were correlated. However, this relationship was weaker for within-subject changes in accent over time. The results suggest that there were limitations in the ability of these subjects to acquire new phonological rules.
2aSC12. Articulatory settings of French-English bilingual speakers. Ian Wilson (Dept. of Linguist., Univ. of British Columbia, 1866 Main Mall, Buchanan E270, Vancouver, BC, Canada V6T 1Z1, ilwilson@ interchange.ubc.ca)

The idea of a language-specific articulatory setting (AS), an underlying posture of the articulators during speech, has existed for centuries [Laver, Historiogr. Ling. 5 (1978)], but until recently it had eluded direct measurement. In an analysis of $\mathrm{x}$-ray movies of French and English monolingual speakers, Gick et al. [Phonetica (in press)] link AS to inter-speech posture, allowing measurement of AS without interference from segmental targets during speech, and they give quantitative evidence showing AS to be language-specific. In the present study, ultrasound and Optotrak are used to investigate whether bilingual English-French speakers have two ASs, and whether this varies depending on the mode (monolingual or bilingual) these speakers are in. Specifically, for inter-speech posture of the lips, lip aperture and protrusion are measured using Optotrak. For inter-speech posture of the tongue, tongue root retraction, tongue body and tongue tip height are measured using optically-corrected ultrasound. Segmental context is balanced across the two languages ensuring that the sets of sounds before and after an inter-speech posture are consistent across languages. By testing bilingual speakers, vocal tract morphology across languages is controlled for. Results have implications for L2 acquisition, specifically the teaching and acquisition of pronunciation.

2aSC13. Paired variability indices in assessing speech rhythm in Spanish/English bilingual language acquisition. Richard Work, Jean Andruski, Eugenia Casielles, Sahyang Kim (Wayne State Univ., Detroit, MI 48202), and Geoff Nathan (Wayne State Univ., Detroit, MI 48202)

Traditionally, English is classified as a stress-timed language while Spanish is classified as syllable-timed. Examining the contrasting development of rhythmic patterns in bilingual first language acquisition should provide information on how this differentiation takes place. As part of a longitudinal study, speech samples were taken of a Spanish/English bilingual child of Argentinean parents living in the Midwestern United States between the ages of $1 ; 8$ and 3;2. Spanish is spoken at home and English input comes primarily from an English day care the child attends 5 days a week. The parents act as interlocutors for Spanish recordings with a native speaker interacting with the child for the English recordings. Following the work of Grabe, Post and Watson (1999) and Grabe and Low (2002) a normalized Pairwise Variability Index (PVI) is used which compares, in utterances of minimally four syllables, the durations of vocalic intervals in successive syllables. Comparisons are then made between the rhythmic patterns of the child's productions within each language over time and between languages at comparable MLUs. Comparisons are also made with the rhythmic patterns of the adult productions of each language. Results will be analyzed for signs of native speaker-like rhythmic production in the child.

2aSC14. Recognition of function words in 8-month-old French-learning infants. Rushen Shi and Bruno Gauthier (Dept. of Psych., Univ. of Quebec in Montreal, C.P. 8888, Succ. Ctr.-Ville, Montreal, QC, Canada H3C 3P8)

Previous work has shown that German-learning 7-9-month-old infants recognize function words (Hoehle and Weissenborn, 2003). Englishlearning infants recognize function words around 10.5-11 months (Schafer et al. 1998; Shady, 1996; Shi et al., 2003, 2004), and the highly frequent determiner "the" at 8 months (Shi et al., 2004). The present study investigates French-learning infants' recognition of function words. As French is a syllable-timing language, the fuller syllabic status may allow infants to recognize function words earlier than English-learning infants. Syntactically and morphologically, functional elements occur more systematically in French than in English, providing reliable statistical cues to functor segmentation. Using a preferential looking procedure, we familiarized 8-month-olds with a target function word ("des," "la," "mes" or "ta"), and tested them with phrases containing the target versus a non-target. 
Results showed that infants' looking time to the phrases containing the targets versus those containing the non-targets differed significantly. Thus, infants recognized the target functors in continuous speech. As the targets included both high-frequency ("des," "la") and low-frequency ("mes," "ta") function words, we suggest that infants may begin segmenting highfrequency functors at an even younger age. The implications of early processing of function words to language acquisition will be discussed.

2aSC15. Bilinguals reaction times and category goodness judgments in two language-sets: Spanish and English. Adrian Garcia-Sierra (Univ. of Texas, CMA A2-2001, Univ. Station A1100, Austin, TX 78712, gasa@austin.utexas.edu)

Two monolingual groups (Spanish and English speakers) and one bilingual group (Mexican-American) were compared in reaction times (RTs) and category goodness $(\mathrm{CG})$ ratings during the identification of a synthetic continuum ranging from $/ \mathrm{ga} /$ to $/ \mathrm{ka} /$. The task consisted of two sessions for the bilingual listeners and one session for the monolingual listeners. Each session was performed in either a Spanish or English language-set. Language-sets were defined by the language used by the researcher and by filler sentences in the language of interest during the identification task (e.g., what do you hear? or Qu fue lo que escuch?). The results show that voicing boundaries were different across all groups including bilinguals in both language-sets (voicing boundary shift). RTs were larger near the voicing boundaries in all groups. Interestingly, bilinguals showed two RT peaks, whereas monolinguals showed only one RT peak. In regard to CG, participants were asked to rate how good exemplar each token was (1 $=$ worst; $5=$ best). Bilinguals rated the synthetic continuum in both language-sets. The scores given by the bilinguals differed in each language-set at VOT values close to the voicing boundary.

2aSC16. Perceived nativeness and sensitivity to temporal adjustments in speech. Yue Wang (Dept of Linguist., Simon Fraser Univ., Burnaby, BC, Canada V5A 1S6, yuew@sfu.ca) and Dawn M. Behne (Norwegian Univ. of Sci. and Technol., NO-7491 Trondheim, Norway)

Native Mandarin Chinese speakers productions of English consonantvowel (CV) syllables have shown syllable-internal temporal adjustments in the direction of native (English)-like CVs (Wang and Behne, 2004). The current study presents two experiments investigating whether these temporal adjustments affect perceived nativeness. For three production types (native-English, Chinese productions of English, native-Chinese), three syllable-internal timing patterns (English-like, Chinese-English-like, Chinese-like) were applied, resulting in nine stimuli types. Native English listeners judged how English-like each stimulus was on a 7-point scale. In the first experiment, production-types and timing patterns were randomized. Results show that listeners can reliably identify nativeness of the three productions, with Chinese productions of English perceived as intermediate to the native Chinese and native American English productions. Listeners also showed a tendency toward using timing within the CV to identify nativeness. In the second experiment the same materials were therefore blocked by production type. Results reveal the perceptual saliency of the temporal adjustments in nonnative productions. These findings support a view of L2 acquisition as a gradual process toward the target L2 (e.g., Caramazza et al., 1973). The current study extends this view, showing evidence that listeners can perceive the inter-language system, bearing the nature of both L1 and L2.

2aSC17. Relation between perception and production ability during a speech training course. Teruaki Tsushima and Mayumi Hamada (Ryutsu Kagaku Univ., 3-1, Gakuen-Nishi-Machi, Nishi-Ku, Kobe, 651-2188, Japan)

Previous research has indicated that perception and production ability are significantly related among L2 learners in natural learning settings. The present study focused on the relation between these two abilities during speech training, specifically examining whether perception or production training alone led to improvements of production or perception ability, and whether the order of perception and production training influenced the improvement of the abilities. Forty-five Japanese university students received thirteen-week perception and production training on six American English contrasts (e.g., b/v, 1/r). One group received perception training first, followed by production training, while the order of training was reversed for the other group. It was found that the initial perception training in the former group resulted in a significant but relatively weak transfer to improvements of production ability, while the initial production training in the latter group showed a significant and strong transfer to those of perception ability. It was also shown that the improvements of perception and production ability during the speech training were significantly related in both groups. The order of training, however, did not significantly influence the overall degree of improvements in either of the abilities. Implications for speech learning models will be discussed.

2aSC18. The effect of $\mathrm{L} 1$ prosodic backgrounds of Cantonese and Japanese speakers on the perception of Mandarin tones after training. Connie K. So (Dept. of Linguist., Simon Fraser Univ., Burnaby, BC, Canada V5A 1S6, klso@sfu.ca)

The present study investigated to what extent ones' L1 prosodic backgrounds affect their learning of a new tonal system. The question as to whether native speakers of a tone language perform differently from those of a pitch accent language will be addressed. Twenty native speakers of Hong Kong Cantonese (a tone language) and Japanese (a pitch accent language) were assigned to two groups. All of them had had no prior knowledge of Mandarin, and had never received any form of musical training before they participated in the study. Their performance of the identification of Mandarin tones before and after a short-term training was compared. Analysis of listeners' tonal confusions in the pretest, posttest, and generalization tests revealed that both Cantonese and Japanese listeners had more confusion for two contrastive tone pairs: Tone 1-Tone 4, and Tone 2-Tone 3. Moreover, Cantonese speakers consistently had greater difficulty than Japanese speakers in distinguishing the tones in each pair. These imply that listeners L1 prosodic backgrounds are at work during the process of learning a new tonal system. The findings will be further discussed in terms of the Perceptual Assimilation Model (Best, 1995). [Work supported by SSHRC.]

2aSC19. How auditory discontinuities and linguistic experience affect the perception of speech and non-speech in English- and Spanishspeaking listeners. Jessica F. Hay (Dept. Psych., Univ. of Texas, Austin, TX 78712, hay@psy.utexas.edu), Lori L. Holt (Carnegie Mellon Univ., Pittsburgh, PA 15213), Andrew J. Lotto (Boys Town Natl. Res. Hospital, Omaha, NE 68131), and Randy L. Diehl (Univ. of Texas, Austin, TX 78712)

The present study was designed to investigate the effects of long-term linguistic experience on the perception of non-speech sounds in English and Spanish speakers. Research using tone-onset-time (TOT) stimuli, a type of non-speech analogue of voice-onset-time (VOT) stimuli, has suggested that there is an underlying auditory basis for the perception of stop consonants based on a threshold for detecting onset asynchronies in the vicinity of $+20 \mathrm{~ms}$. For English listeners, stop consonant labeling boundaries are congruent with the positive auditory discontinuity, while Spanish speakers place their VOT labeling boundaries and discrimination peaks in the vicinity of $0 \mathrm{~ms}$ VOT. The present study addresses the question of whether long-term linguistic experience with different VOT categories affects the perception of non-speech stimuli that are analogous in their acoustic timing characteristics. A series of synthetic VOT stimuli and TOT stimuli were created for this study. Using language appropriate labeling and $\mathrm{ABX}$ discrimination tasks, labeling boundaries (VOT) and discrimination peaks (VOT and TOT) are assessed for 24 monolingual English speakers and 24 monolingual Spanish speakers. The interplay between language experience and auditory biases are discussed. [Work supported by NIDCD.] 
2aSC20. Method for automatic measurement of second language speaking proficiency. Jared Bernstein and Jennifer Balogh (Ordinate Corp., Menlo Park, CA 94025)

Spoken language proficiency is intuitively related to effective and efficient communication in spoken interactions. However, it is difficult to derive a reliable estimate of spoken language proficiency by situated elicitation and evaluation of a person's communicative behavior. This paper describes the task structure and scoring logic of a group of fully automatic spoken language proficiency tests (for English, Spanish and Dutch) that are delivered via telephone or Internet. Test items are presented in spoken form and require a spoken response. Each test is automatically-scored and primarily based on short, decontextualized tasks that elicit integrated listening and speaking performances. The tests present several types of tasks to candidates, including sentence repetition, question answering, sentence construction, and story retelling. The spoken responses are scored according to the lexical content of the response and a set of acoustic base measures on segments, words and phrases, which are scaled with IRT methods or parametrically combined to optimize fit to human listener judgments. Most responses are isolated spoken phrases and sentences that are scored according to their linguistic content, their latency, and their fluency and pronunciation. The item development procedures and item norming are described.

2aSC21. Effects of linguistic experience on early levels of perceptual tone processing. Tsan Huang (Dept. of Linguist., SUNY Buffalo, 609 Baldy Hall, Buffalo, NY 14260) and Keith Johnson (UC Berkeley, Berkeley, CA 94720-2650)

This study investigated the phenomenon of language-specificity in Mandarin Chinese tone perception. The main question was whether linguistic experience affects the earliest levels of perceptual processing of tones. Chinese and American English listeners participated in four perception experiments, which involved short inter-stimulus intervals $(300 \mathrm{~ms}$ or $100 \mathrm{~ms}$ ) and an AX discrimination or AX degree-of-difference rating task. Three experiments used natural speech monosyllabic tone stimuli and one experiment used time-varying sinusoidal simulations of Mandarin tones. $\mathrm{AE}$ listeners showed psychoacoustic listening in all experiments, paying much attention to onset and offset pitch. Chinese listeners showed language-specific patterns in all experiments to various degrees, where tonal neutralization rules reduced perceptual distance between two otherwise contrastive tones for Chinese listeners. Since these experiments employed procedures hypothesized to tap the auditory trace mode (Pisoni, Percept. Psychophys. 13, 253-260 (1973)], language-specificity found in this study seems to support the proposal of an auditory cortical map [Guenther et al., J. Acoust. Soc. Am. 23, 213-221 (1999)]. But the model needs refining to account for different degrees of language-specificity, which are better handled by Johnsons (2004, TLS03:26-41) lexical distance model, although the latter model is too rigid in assuming that linguistic experience does not affect low-level perceptual tasks such as AX discrimination with short ISIs.

2aSC22. Perceptual and production variables in explicating interlanguage speech intelligibility benefit. Amee $P$. Shah and Zoi Vavva (Dept. of Speech and Hearing, Cleveland State Univ., 2121 Euclid Ave., MC 431-B, Cleveland, OH 44115, a.shah101@ csuohio.edu)

This study attempts to investigate the importance of the degree of similarity or difference in the language backgrounds of the speakers and listeners, as it interacts differentially in intelligibility judgment of foreignaccented speech (Bent and Bradlow, 2003). The present study attempts to clarify the distinction in the matched and mismatched listening conditions, in context of addressing the overarching question whether auditory exposure to a language alone, without corresponding proficiency in production of that language, can provide a listening advantage. Particularly, do listeners understand accented-English speech spoken by native individuals of the language to which they are exposed to, as compared to listeners without that exposure? Greek-accented English speakers (and native monolin- gual English speakers) were judged for their speech intelligibility by four groups of listeners $(n=10$, each): native Greek speakers (matched), Greek-Americans (matched only through auditory exposure to Greek without any corresponding spoken proficiency), native monolingual AmericanEnglish speakers (unmatched), and a mixed group (mismatched). Pilot data have shown that the intelligibility judgments by Greek-American listeners are intermediate to the native Greeks, and both the AmericanEnglish and the mixed group. Further data-collection is underway, and will be presented as they bear important theoretical and clinical implications.

2aSC23. Phonological systems in bilinguals: Age of learning effects on the stop consonant systems of Korean-English bilinguals. Kyoung-Ho Kang and Susan G. Guion (Dept. of Linguist., 1290 Univ. of Oregon, Eugene, OR 97403, kkang@darkwing.uoregon.edu)

The stop systems of adult Korean (L1)-English (L2) bilinguals were studied through acoustic analysis of Korean and English stop productions. The interaction of the first and second language stop systems was investigated as a function of age of exposure to English. The main goals of the investigation were to examine the extent to which early and the late bilinguals produced a given stop category in a native-like way and the extent to which the two stop systems were held independent from each other in the phonological systems of the bilinguals. Two specific questions were asked: whether early bilinguals were more native-like in the production of English stops and thus were more likely to establish L2 phonetic categories than late bilinguals, and whether the Early bilinguals maintain a greater extent of independence between the Korean and English stop systems than the late bilinguals. For this purpose, productions of Korean and English stops were analyzed in terms of three acoustic-phonetic properties, VOT, $H 1-H 2$, and $f 0$. The results suggested that fine-grained phonetic information is crucial to investigation of bilingual phonological systems and they were discussed in terms of the role of perceived phonetic distance between L1 and L2 phonetic categories in L2 speech learning.

2aSC24. Validation of an automatic measurement of Spanish speaking proficiency. Elizabeth Rosenfeld, Jared Bernstein, and Jennifer Balogh (Ordinate Corp., Menlo Park, CA)

A 15-min computer-based test of spoken Spanish was designed to measure candidate proficiency in Spanish. The test presents seven tasks: reading, elicited imitation, word opposites, short-answer questions, sentence constructions, opinion questions, and story retellings. The tests were presented to 579 adult non-native Spanish learners and to 552 native Spanish speakers. Expert human judgments of the non-native responses showed that the spoken response material carried sufficient information for highly reliable judgments of proficiency. In the development and validation process, 57000 responses were transcribed and 21000 human judgments were analyzed. The paper describes the validation of the automatic scoring system with reference to concurrent oral proficiency interviews conducted by professional raters certified by the US Government or by ACTFL. The outcomes of the comparisons of the machine scored tests with interactive human interviews and with human ratings from recorded speech indicate that the test produces scores that have virtually the same information that is found in oral proficiency interviews. Almost all assessments correlate highly with the other assessments with coefficients in the range $0.86-0.96$. The test correlation with the combined interview scores $(r=0.92)$ is higher than the inter-rater reliability of the professional interviewers themselves.

2aSC25. An acoustic investigation of the Cantonese vowels in the speech of the adult and child speakers. Wai-Sum Lee (Dept. of Linguist., The Univ. of Hong Kong, Pokfulam Rd., Hong Kong, wsleeba@ hku.hk)

The study analyzes the formant center frequencies for the seven Cantonese vowels [i, y, u, $\varepsilon, \alpha, \rho, \mathrm{a}]$ from 30 native speakers of Cantonese, 10 male and 10 female adults and 5 male and 5 female 9-10 year old children. Results show that the formant frequencies for the vowels are 
largest for the female children, followed by the male children, female adults, and male adults in decreasing order. Despite the differences, the patterns of formant frequencies for any one vowel for the different groups are similar. The difference in $F$-values for any one vowel between the male and female children is smaller than the difference between the male and female adults. As for individual formant frequencies, the difference in $F 1$ between the males and females of the same age group and between the adults and children of the same gender group is smaller for the high vowels $[\mathrm{i}, \mathrm{y}, \mathrm{u}]$ than the non-high vowels $[\varepsilon, \propto, \rho, \mathrm{a}]$. The difference in $F 2$ between the males and females of the same age group and between the adults and children of the same gender group is smaller for the high rounded vowels $[\mathrm{y}, \mathrm{u}]$ than the other vowels. The paper will also present the ratios of speaker group-to-speaker group for individual formant frequencies.

2aSC26. Voice onset time (VOT) in Canadian French and English: Monolingual and bilingual adults. Andrea A. N. MacLeod and Carol Stoel-Gammon (Dept. of Speech and Hearing Sci., Univ. of Washington, 1417 N.E. 42nd St., Seattle, WA 98119)

This study focused on the contrasts produced by early bilingual speakers $(n=6)$ across their two languages in comparison with monolingual speakers (Canadian English (CE), $n=5$; Canadian French (CF), $n=6$ ). VOT production was measured in monosyllabic $\mathrm{CE}$ and $\mathrm{CF}$ words that began with one of four stop consonants, /p, b, t, d/ followed by one of three vowels. A total of 14-18 words for each of the four stop consonants for each language was elicited with a total number 1700 acoustically analyzed productions. The participants were tested individually in quiet rooms using a single target language throughout the session. As expected, the monolingual speakers produced a two-way contrast (statistically significant: $p<0.05$ ): for CE speakers, short-lag VOT versus long-lag VOT; for CF speakers, lead VOT versus short-lag VOT. Rather than producing a two-way contrast (e.g., lead VOT versus lag VOT) or a three-way contrast (e.g., lead VOT versus short-lag VOT versus long-lag VOT), the bilingual speakers produced a four-way contrast (statistically significant: $p$ $<0.05)$ : long lead VOT (CF /b, d/), short lead VOT (CE /b, d/), short-lag $\operatorname{VOT}(\mathrm{CF} / \mathrm{p}, \mathrm{t} /)$ and long-lag VOT $(\mathrm{CE} / \mathrm{p}, \mathrm{t} /)$. These results suggest that bilinguals are maintaining phonetic contrasts both within and across their two languages.

2aSC27. The effect of speaking rate on perception of syllables in second-language speech. Keiichi Tajima (Dept. of Psych., Hosei Univ., Tokyo 102-8160, Japan; ATR Human Information Sci. Labs., Kyoto 619-0288, Japan) and Reiko Akahane-Yamada (ATR Human Information Sci. Labs., Kyoto 619-0288, Japan)

Past studies on second-language (L2) speech perception have suggested that L2 learners have difficulty exploiting contextual information when perceiving L2 utterances, and that they exhibit greater difficulty than native listeners when faced with variability in temporal context. The present study investigated the extent to which native Japanese listeners, who are known to have difficulties perceiving English syllables, are influenced by changes in speaking rate when asked to count syllables in spoken English words. The stimuli consisted of a set of English words and nonwords varying in syllable structure spoken at three rates by a native English speaker. The stimuli produced at the three rates were presented to native Japanese listeners in a random order. Results indicated that listeners' identification accuracy did not vary as a function of speaking rate, although it decreased significantly as the syllable structure of the stimuli became more complex. Moreover, even though speaking rate varied from trial to trial, Japanese listeners' performance did not decline compared to a condition in which the speaking rate was fixed. Theoretical and practical implications of these findings will be discussed. [Work supported by JSPS and NICT.]
2aSC28. Learning to talk: A non-imitative account of the replication of phonetics by child learners. Piers Messum (Dept. of Phonet. and Linguist., UCL, Gower St., London WC1E 6BT, UK)

How is it that an English-speaking 5-year-old comes to: pronounce the vowel of seat to be longer than that of sit, but shorter than that of seed; say a multi-word phrase with stress-timed rhythm; aspirate the $/ \mathrm{p} / \mathrm{s}$ of pin, polite, and spin to different degrees? These are systematic features of English, and most people believe that a child replicates them by imitation. If so, he is paying attention to phonetic detail in adult speech that is not very significant linguistically, and then making the effort to reproduce it. With all the other communicative challenges he faces, how plausible is this? An alternative, non-imitative account of the replication of these features relies on two mechanisms: (1) emulation, and (2) the conditioning of articulatory activity by the developing characteristics of speech breathing. The phenomena above then become no more than expressions of how a child finds ways to warp his phonetic output in order to reconcile conflicting production demands. The criteria he uses to do this make the challenges both of learning to talk and then of managing the interaction of complex phonetic patterns considerably more straightforward than has been imagined.

2aSC29. Acoustics of contrastive prosody in children. Rupal Patel, Jordan Piel (Dept. of Speech Lang. Pathol. \& Audiol., Northeastern Univ., 360 Huntington Ave., Boston, MA 02115, r.patel@neu.edu), and Maria Grigos (New York Univ., New York, NY 10003)

Empirical data on the acoustics of prosodic control in children is limited, particularly for linguistically contrastive tasks. Twelve children aged 4, 7, and 11 years were asked to produce two utterances "Show Bob a bot" (voiced consonants) and "Show Pop a pot" (voiceless consonants) 10 times each with emphasis placed on the second word (Bob/Pop) and 10 times with emphasis placed on the last word (bot/pot). A total of 40 utterances were analyzed per child. The following acoustic measures were obtained for each word within each utterance: average fundamental frequency $(f 0)$, peak $f 0$, average intensity, peak intensity, and duration. Preliminary results suggest that 4 year olds are unable to modulate prosodic cues to signal the linguistic contrast. The 7 year olds, however, not only signaled the appropriate stress location, but did so with the most contrastive differences in $f 0$, intensity, and duration, of all age groups. Prosodic differences between stressed and unstressed words were more pronounced for the utterance with voiced consonants. These findings suggest that the acoustics of linguistic prosody begin to differentiate between age 4 and 7 and may be highly influenced by changes in physiological control and flexibility that may also affect segmental features.

2aSC30. Infant-directed speech: Final syllable lengthening and rate of speech. Robyn Church, Barbara Bernhardt (School of Audiol. and Speech Sci., Univ. of British Columbia, Vancouver, BC, Canada V6T 1Z1), Rushen Shi (Univ. of Quebec at Montreal), and Kathleen Pichora-Fuller (Univ. of Toronto)

Speech rate has been reported to be slower in infant-directed speech (IDS) than in adult-directed speech (ADS). Studies have also found phrase-final lengthening to be more exaggerated in IDS compared with ADS. In our study we asked whether the observed overall slower rate of IDS is due to exaggerated utterance-final syllable lengthening. Two mothers of preverbal English-learning infants each participated in two recording sessions, one with her child, and another with an adult friend. The results showed an overall slower rate in IDS compared to ADS. However, when utterance-final syllables were excluded from the calculation, the speech rate in IDS and ADS did not differ significantly. The duration of utterance-final syllables differed significantly for IDS versus ADS. Thus, the overall slower rate of IDS was due to the extra-long final syllable occurring in relatively short utterances. The comparable pre-final speech rate for IDS and ADS further accentuates the final syllable lengthening in IDS. As utterances in IDS are typically phrases or clauses, the particularly 
strong final-lengthening cue could potentially facilitate infants' segmentation of these syntactic units. These findings are consistent with the existing evidence that pre-boundary lengthening is important in the processing of major syntactic units in English-learning infants.

2aSC31. Neural-network simulation of tonal categorization based on F 0 velocity profiles. Bruno Gauthier, Rushen Shi (Dept. of Psych., Univ. of Quebec in Montreal, C.P. 8888, Succ. Centre-ville, Montreal, QC, Canada H3C 3P8), Yi Xu (Univ. College London, London, NW1 2HE, UK), and Robert Proulx (Univ. of Quebec in Montreal, Montreal, QC, Canada H3C 3P8)

Perception studies have shown that by the age of six months, infants show particular response patterns to tones in their native language. The present study focuses on how infants might develop lexical tones in Man- darin. $F 0$ is generally considered the main cue in tone perception. However, $F 0$ patterns in connected speech display extensive contextual variability. Since speech input to infants consists mainly of multi-word utterances, tone learning must involve processes that can effectively resolve variability. In this study we explore the Target Approximation model (Xu and Wang, 2001) which characterizes surface $F 0$ as asymptotic movements toward underlying pitch targets defined as simple linear functions. The model predicts that it is possible to infer underlying pitch targets from the manners of $F 0$ movements. Using production data of three of the speakers from Xu (1997), we trained a self-organizing neural network with both $F 0$ profiles and $F 0$ velocity profiles as input. In the testing phase, velocity profiles yielded far superior categorization than $F 0$ profiles. The results confirm that velocity profiles can effectively abstract away from surface variability and directly reflect underlying articulatory goals. The finding thus points to one way through which infants can successfully derive at phonetic categories from adult speech.

TUESDAY MORNING, 17 MAY 2005

GEORGIA A, 8:00 TO 11:45 A.M.

\title{
Session 2aSP
}

\section{Signal Processing in Acoustics: Stochastic Signal Processing and Inversion}

\author{
Max Deffenbaugh, Cochair \\ Exxon Mobil Upstream Research, P.O. Box 2189, Houston, TX 77252 \\ Alan W. Meyer, Cochair \\ Lawrence Livermore National Lab., Univ. of California, 700 East Ave., Livermore, CA 94550 \\ Chair's Introduction-8:00 \\ Invited Papers
}

8:05

2aSP1. Signal processing techniques for inverse problems in stochastic propagation and scattering channels. Leon H. Sibul, Michael J. Roan, and Christian M. Coviello (Appl. Res. Lab., Penn State Univ., P.O. Box 30, State College, PA 16804-0030, 1hs2@ psu.edu)

The basic signal processing techniques associated with inverse problems are signal extraction, deconvolution, signal and signal parameter estimation, channel modeling and characterization. Maximum entropy, minimum cross-entropy, Kullback-Liebler divergence and other information theoretic criteria have been widely used for regularization of underdetermined inversion. We show how maximum entropy and continuous wavelet transforms can be used for spreading function (reflection density function) estimation. If the signal source or receivers are in motion through heterogeneous medium with randomly rough boundaries, propagation and scattering channels are stochastic. Stochastic channels can be characterized by stochastic Greens functions and spreading functions. If the medium probing signals are narrow band, spreading functions are random functions that show how the received signal is spread in delay and Doppler. For wideband probing signals, spreading function spread the received echo in time and time-scale dilation. If the probing signal satisfies admissibility conditions for CWT, wideband spreading functions can be estimated by inverting CWT. Rebollo-Neira and Fernandez-Rubio have shown that the continuous wavelet transform is an optimal solution of the inverse problem, estimation of the spreading function, in a maximum entropy sense. [Work supported by Office of Naval Research Code 333 and Code 321 US.]

8:30

2aSP2. Quantifying heterogeneity: Attributes, modeling, and inversion. Matthias Georg Imhof (Dept. of Geosciences, Virginia Tech, Blacksburg, VA 24061)

Characterization of reservoir heterogeneity is a necessary step in reservoir delineation, characterization, and modeling. Reservoir heterogeneity can be described deterministically, statistically, or with reservoir-forming geologic processes. Deterministic heterogeneity models are easy to build, but may have insufficient resolution and may not provide enough insights into the reservoir and its properties. The parameterization of stochastic heterogeneity models is nontrivial. Seismic data can be used to determine geostatistical parameters or to refine the geometry parameters used for Boolean modeling. The geostatistical parameters (ranges and orientations) are obtained from seismic heterogeneity attributes measuring the second-order statistics contained in small seismic datacubes. Seismic heterogeneity relates to acquisition and processing artifacts, structure, or stratigraphy and lithology. Seismic data could also be used as additional constraints in Boolean reservoir models which allows both construction of conforming reservoir models and optimization 
of the geometry parameters to reduce the misfit between model and observations. Reservoirs could also be built by reconstructing their formation and evolution based on mathematical descriptions of processes such as sediment erosion, transport and deposition, compaction, deformation, subsidence and uplift, etc. The process parameters, e.g., rates of sediment input and transport, compaction, or subsidence, could be estimated by inversion of seismic and geologic data.

8:55

2aSP3. Sparseness- and continuity-constrained seismic imaging. Felix J. Herrmann (Dept. of Earth and Ocean Sci., Univ. of British Columbia, 6339 Stores Rd., Vancouver, BC, Canada V6T 1Z4, fherrmann@eos.ubc.ca)

Non-linear solution strategies to the least-squares seismic inverse-scattering problem with sparseness and continuity constraints are proposed. Our approach is designed to (i) deal with substantial amounts of additive noise (SNR $<0 \mathrm{~dB}$ ); (ii) use the sparseness and locality (both in position and angle) of directional basis functions (such as curvelets and contourlets) on the model: the reflectivity; and (iii) exploit the near invariance of these basis functions under the normal operator, i.e., the scattering-followed-by-imaging operator. Signal-to-noise ratio and the continuity along the imaged reflectors are significantly enhanced by formulating the solution of the seismic inverse problem in terms of an optimization problem. During the optimization, sparseness on the basis and continuity along the reflectors are imposed by jointly minimizing the $l^{1}$ - and anisotropic diffusion/total-variation norms on the coefficients and reflectivity, respectively. [Joint work with Peyman P. Moghaddam was carried out as part of the SINBAD project, with financial support secured through ITF (the Industry Technology Facilitator) from the following organizations: BG Group, BP, ExxonMobil, and SHELL. Additional funding came from the NSERC Discovery Grants 22R81254.]

9:20

2aSP4. Reconciling data using Markov chain Monte Carlo: An application to the Yellow Sea-Korean Peninsula region. Michael Pasyanos (Lawrence Livermore Natl. Lab., 7000 East Ave., Livermore, CA 94550)

We present a technique for developing seismic models using multiple data sets using a probabilistic inverse technique. A Markov Chain Monte Carlo (MCMC) algorithm is used to sample models from a prior distribution and test them against multiple data types to generate a posterior distribution. This approach has several advantages over deterministic models, notably the reconciliation of different data types that constrain the model, the proper handling of uncertainties, and the ability to include prior information. We also benefit from the advantage of forward modeling rather than inverting the data. We demonstrate this method by using it to determine the 3-D crust and upper mantle structure for the Yellow Sea and Korean Peninsula (YSKP) region. The model is parameterized as a series of layers in a regular lat-lon grid. We start with an a priori model and use surface wave dispersion measurements, body wave travel times, teleseismic receiver functions, and gravity data to drive the model. The end result is a robust model distribution for the region that is best able to fit multiple data sets, and is consistent with our understanding of the tectonics of the region.

9:45

2aSP5. Global seismic tomography: Present status and future perspectives. Barbara Romanowicz (Berkeley Seismological Lab., 215 McCone Hall, Berkeley, CA 94720)

Global seismic tomography of the Earth's mantle has traditionally focused on the inversion of travel times from a small number of seismic phases, well isolated in time domain seismograms. The resulting uneven sampling of the Earth, dictated by the available distribution of earthquake sources and receivers, greatly limits the resolution of 3-D structure that can be attained. With recent progress in the simulation of wave propagation in realistic 3-D Earth models combined with significant increases in computational power, seismologists are well positioned to start fully exploiting the wealth of information contained in the rapidly expanding database of high quality broadband seismic waveforms, accumulated globally and regionally over the last twenty five years. New opportunities presented by 3-D forward and inverse modeling of long period waveforms will be presented with illustrations from recent work on deep and shallow elastic and anelastic mantle structure, addressing, among others, such questions as: what is the fate of subducted slabs or the nature of the lower mantle "superplumes"?

10:10-10:30 Break

10:30

2aSP6. Surface wave tomography from seismic ambient noise in Southern California. Karim Sabra, Peter Gerstoft, Philippe Roux, Willliam Kuperman (Marine Physical Lab., Scripps Inst. of Ocean., UCSD, La Jolla, CA 92093-0238, ksabra@ mpl.ucsd.edu), and Michael Fehler (Los Alamos Natl. Lab., NM)

It has been demonstrated experimentally that an estimate of the Green's tensor between two seismic stations can be obtained from the long-time average of the cross-correlation of ambient noise at the two stations. This result provides a means to image Earth structure using the ambient noise field only, without the use of active seismic sources or earthquakes. Seismic noise data from 148 broadband seismic stations in Southern California were used to extract the surface wave arrival-times between all station pairs in the network in the frequency band $0.05-0.4 \mathrm{~Hz}$. In this frequency band, ambient noise (originating from ocean microseisms) propagating over long distances is typically dominated by surface waves. A record section of the waveforms as a function of increasing receiver separation shows clearly that the recovered signals are propagating wavetrains. The seismic data were then used in a simple, but densely sampled tomographic procedure to estimate the surface wave velocity structure for a region in Southern California. The result compares favorably with previous estimates obtained using more conventional and elaborate inversion procedures. This demonstrates that coherent ambient noise between station pairs can be used for seismic imaging purposes. 
2aSP7. An overview of geophysical investigations at archaeological sites in Helike, Greece. Alan Witten and Jamie Rich (School of Geology and Geophys., Univ. of Oklahoma, 810 Energy Ctr., Norman, OK 73019)

Helike is an area on the south-central coast of the Gulf of Corinth. This name is derived from the lost Classical Greek city of Helike that vanished during an earthquake and subsequent tsunami in $373 \mathrm{BC}$. It was original believed that the remains of Helike were located offshore in the Gulf of Corinth; however, early searches of this area proved fruitless. More recently, boreholes developed in the coastal plain revealed marine sediments that date to the Classical Greek period suggesting that, as a result of uplift and/or sedimentation, Helike now lies onshore. This find stimulated a sequence of ongoing geophysical investigations and excavations that have revealed buried architecture and artifacts from three periods; Early Bronze Age, Classical Greek, and Roman.

11:20

2aSP8. Acoustic archeology at the lost city of Helike. Sean K. Lehman (Lawrence Livermore Natl. Lab., L-154, 7000 East Ave., Livermore, CA 94550)

In $373 \mathrm{BC}$ an earthquake and tsunami destroyed and submerged the classical Greek city of Helike (HELL-E-KEY) on the north Peloponnese shore of the Gulf of Corinth. In June 2004, surface seismo-acoustic surveys were performed in the area of present Helike in order to collect non-invasive tomographic data to guide the archeological excavations. Prior to tomographic inversion and imaging, the raw data time series must be preprocessed to estimate ground acoustic velocity, and to remove the ground roll signal. We present a generalized cross-correlation technique which appears successful in solving both problems. We describe the technique as applied to the data and present tomographic inversions.

TUESDAY MORNING, 17 MAY 2005

GEORGIA B, 7:40 A.M. TO 12:00 NOON

\title{
Session 2aUW
}

\section{Underwater Acoustics and Acoustical Oceanography: Acoustic Interaction with Ocean Boundaries: Single Bounce Measurements}

\author{
Charles W. Holland, Chair \\ Applied Research Lab., Pennsylvania State Univ., State College, PA 16804-0030
}

Chair's Introduction-7:40

Contributed Papers

\section{$7: 45$}

2aUW1. Direct-path rough surface scattering experiments with milled surfaces in acoustic tank facilities. Raymond J. Soukup, Robert F. Gragg, Jason E. Summers, and Edward L. Kunz (Naval Res. Lab., 4555 Overlook Ave., Washington, DC 20375, soukup@abyss.nrl.navy.mil)

To investigate specific issues relating to rough surface scattering theoretical predictions, the Naval Research Laboratory has been conducting scaled-down versions of its direct-path ocean bottom scattering experiments. These experiments utilize milled elastic rough surfaces in acoustic tank facilities. Investigations into the dependence of scattering on roughness and geoacoustic parameters lend themselves to a systematic treatment with milled surfaces that are fabricated to match specific roughness spectral parameters. The characterization and fabrication of such rough surfaces, and the methodology for creating experimental geometries that are analogous to previous measurements with elastic ocean bottoms, are described. A set of scattering strength measurements in the $100-300 \mathrm{kHz}$ band with a quasi-monostatic geometry is compared with the theoretical predictions given by perturbation theory and the NRL small slope model. The results show that these experiments are a viable means of verifying the predictions of the scattering models, given a sufficiently large statistical sample. [Work supported by ONR.]
8:00

2aUW2. Observations of a geoclutter feature in the straits of Sicily. Thomas C. Weber, Charles W. Holland (Appl. Res. Lab., The Pennsylvania State Univ., State College, PA 16804), and Giuseppe Etiope (Natl. Inst. of Geophys. and Volcanology, Rome, Italy)

Several persistent sonar clutter features were observed in 2002 during an experiment in the Straits of Sicily (Malta Plateau) in a region that is nominally flat and thickly sedimented. High frequency sidescan and seismic reflection data from the region indicated that mud volcanoes were present and were possibly expelling clouds of methane bubbles. In 2004 the region was revisited with oceanographic equipment including a multibeam sonar, an acoustic Doppler current profiler (ADCP), CTD's, temperature loggers, a methane sensor, and a video camera. The visual observations at one site show carbonate mounds comprised of large heterogeneous blocks that suggest the presence of mud volcanism. A multibeam sonar survey of the area shows that the carbonate mounds are of order 5-10 $\mathrm{m}$ in height and $10-100 \mathrm{~m}$ in lateral dimension, and that they form in clusters. The multibeam backscatter data show high amplitudes in the region surrounding the carbonate mounds, possibly indicating the surficial extent of the carbonate material. High levels of methane were observed in the water column above the mounds, although conclusive evidence of the presence of methane bubble clouds was not found. However, high frequency backscatter recorded on the ADCP above multiple 
carbonate mounds showed plume-like features that may be bubble clouds, suspended fine-grained sediment, and/or schools of fish.

8:15

2aUW3. Close-range acoustic scattering from mud volcanoes. Charles W. Holland, Thomas C. Weber (Appl. Res. Lab., Penn State Univ., State College, PA, 16804), and Giuseppe Etiope (Istituto Nazionale di Geofisica e Vulcanologia, Rome, Italy)

Submarine mud volcanoes occur in many parts of the world's oceans and form an aperture for gas (mostly methane) and fluidized mud emission from the earth's interior. Their characteristics are of considerable interest to the geology, geophysics, geochemistry, and underwater acoustics communities. For the later community, mud volcanoes are important because they pose a potential source of clutter for active sonar. Close-range (single boundary interaction) scattering measurements from a mud volcano in the Straits of Sicily were conducted with a vertical source and receive array. The data show target strengths from $800-3600 \mathrm{~Hz}$ of $6-12 \mathrm{~dB}$ for a monostatic geometry with grazing angles of 3-5 degrees. The target strengths are very similar for vertically bi-static paths with incident grazing angles of 3-5 degrees and scattered angles of 33-45 degrees. The evidence suggests that the scattering mechanism is the mud volcano (carbonate) structure. [Work supported by the Office of Naval Research and NATO Undersea Research Centre.]

8:30

2aUW4. Comparison of the magnitude and phase of the reflection coefficient from a smooth water/sand interface with elastic and poroelastic models. Marcia Isakson (Appl. Res. Labs., Univ. of Texas, Austin, TX 78713-8029), H. John Camin (The Penn State Univ., State College, PA 16804-0030), and Gaetano Canepa (NATO Underea Res. Ctr., La Spezia, Italy)

The reflection coefficient from a sand/water interface is an important parameter in modeling the acoustics of littoral environments. Many models have been advanced to describe the influence of the sediment parameters and interface roughness parameters on the reflection coefficient. In this study, the magnitude and phase of the reflection coefficient from 30 to $160 \mathrm{kHz}$ is measured in a bistatic experiment on a smoothed water/sand interface at grazing angles from 5 to 75 degrees. The measured complex reflection coefficient is compared with the fluid model, the elastic model and poro-elastic models. Effects of rough surface scattering are investigated using the Bottom Response from Inhomogeneities and Surface using Small Slope Approximation (BoRIS-SSA). Spherical wave effects are modeled using plane wave decomposition. Models are considered for their ability to predict the measured results using realistic parameters. [Work supported by ONR, Ocean Acoustics.]

\section{$8: 45$}

2aUW5. Laboratory study of high frequency scattering from watersaturated sandy sediments. Anatoliy Ivakin (Appl. Phys. Lab., Univ. of Washington, 1013 NE 40th St., Seattle, WA 98105, ivakin@ apl.washington.edu), Jean-Pierre Sessarego, and Patric Sanchez (CNRS/ LMA, Marseille cedex 20, France)

Backscattering from a water-saturated well-sorted fine sand and from this sand with various inclusions was studied in a laboratory tank at frequencies from $200 \mathrm{kHz}$ to $3 \mathrm{MHz}$ and grazing angles from 20 to 90 degrees. Acoustic and granulometric properties of the sand are presented and discussed in a companion paper. First, the measurements of the frequency-angular dependencies of the backscattering strength were made for a thick sediment layer with a smoothed surface and without any inclusions in the volume. Then glass beads and coarse sand particles were used as additional scatterers or inclusions placed on the sediment surface or covered by sediment layers of various thickness. The data were compared with a previously reported model of scattering from discrete inclusions in the sediment [A. N. Ivakin, J. Acoust. Soc. Am. 116, 2575 (2004)]. The model inputs are material parameters of the sediment and inclusions and the size-depth distribution of inclusions. The results of model/data comparison are discussed and possibilities for inversion of various sediment parameters from backscattering data are shown.

\section{9:00}

2aUW6. Theory and experiments for surface-wave focused acoustic arrivals: A deterministic view. Grant Deane (Mail Code 0238, Scripps Inst. of Oceanogr., UCSD, La Jolla, CA 92093-0238) and James Preisig (Woods Hole Oceanogr. Inst., Woods Hole, MA 02543)

Statistical descriptions of surface wave scattering are now highly developed, and have proven very effective in relating the first and second order statistics of surface-reflected acoustic arrivals to surface conditions. Notwithstanding this success, the deterministic features of surfacescattered arrivals are of considerable importance. The limits of performance of underwater communications systems in very shallow water, for example, are determined by the frequency and properties of extreme surface focusing events. The analysis of data from the Wavefronts I, II, and V series of experiments has led to an understanding of the wave properties controlling the focusing events, as well as their impact on acoustic channel estimators. Data from the wavefronts experiments (SIO pier, $7 \mathrm{~m}$ deep, 40 $\mathrm{m}$ range) and the SPACE02 experiment (Martha's Vineyard, $15 \mathrm{~m}$ depth, 250-1000 m range) will be presented and discussed. [Work supported by ONR.]

\section{$9: 15$}

2aUW7. Forward scattering from the sea surface: Observations of both subtle and profound effects of bubbles in single-interaction measurements. Peter H. Dahl (Appl. Phys. Lab., Univ. of Washington, Seattle, WA 98105)

For frequencies of $\mathrm{O}(10) \mathrm{kHz}$ and above, field data show that nearsurface bubbles impact forward scattering from the sea surface in three phases. The first occurs under mild conditions (wind speed less than 5-7 $\mathrm{m} / \mathrm{s}$ ); here a pulse forward scattered from the sea surface is extended in time, but only at levels $\sim 30 \mathrm{~dB}$ below the peak level, which itself is not attenuated. The second occurs under more vigorous conditions (wind speed $7-12 \mathrm{~m} / \mathrm{s}$ ); here a significant energy loss is observed, but time and angle spreading (dominated by rough surface scattering) remain relatively unchanged. The third occurs under still more vigorous conditions (wind speed greater than $\sim 12 \mathrm{~m} / \mathrm{s}$ ). Here, there is near total occlusion of the sea surface, time and angle spreading are manifestly altered, and bubblemediated energy loss becomes bounded by scattering from bubbles. Examples from ASIAEX East China Sea and other archival data sets will be discussed along with a model for bubble-mediated energy loss in forward scattering from the sea surface. In the case of near total occlusion, an interesting example of the knock-down of horizontal coherence will be discussed along with a model that utilizes the van Cittert-Zernike Theorem. [Research supported by ONR Ocean Acoustics.]

$$
\text { 9:30 }
$$

2aUW8. On the relationship between sea state and the coherent-toincoherent intensity ratio for high frequency, shallow water propagation. Philippe Roux, William A. Kuperman (Marine Physical Lab., Scripps Inst. of Oceanogr., UCSD, 8820 Shellback Way, La Jolla, CA 92093-0238), R. Lee Culver, Steven D. Lutz, David L. Bradley (State College, PA 16804, rlc5@psu.edu), and Mark Stevenson (NATO Undersea Res. Ctr., La Spezia, Italy)

A 29-element source array and 32-element receiver array have been deployed in shallow water to investigate various aspects of the acoustic channel. Both arrays spanned much of the water column. Source-receiver range was $800 \mathrm{~m}$, and $3.5 \mathrm{kHz}$ pulses were transmitted using one source at a time and recorded on all elements. In this analysis, we compare the ratio of coherently to incoherently averaged intensities for each source-receiver pair, and averaged over all such pairs, to measure surface roughness. We find that the coherent-to-incoherent intensity ratio decreases with time after the direct path arrival, and that the slope becomes more negative as 
wind speed and thus surface roughness increases. A simple model for signal phase variation induced by surface scatter shows a direct relationship between the degree of phase variation and wind speed. The relationship between the slope of the coherent-to-incoherent intensity ratio and a physics-based model of incoherent sea surface forward scatter is also considered. [Work supported by ONR Code 321US.]

\section{9:45}

2aUW9. Scintillation index of ocean surface forward scattered HF acoustic signals: Beam pattern and pulse length effects. Benjamin Cotté, David Bradley, and R. Lee Culver (Appl. Res. Lab., Penn State Univ., State College, PA 16804, bzc125@psu.edu)

The intensity fluctuations of surface bounce path measured during a field test near San Clemente Island in August 2002 have been presented previously. Signals used during this test were $20-$ and $40-\mathrm{kHz}$ continuous wave $(\mathrm{CW})$ pulses with 0.14- and 1.0-ms durations ("short" and "long" pulses, respectively). Experimental results have been compared to predictions of a model by Yang and McDaniel [Waves Random Media, 1, 419439 (1991); J. Acoust. Soc. Am. 91, 1960-1966 (1992)]. Their initial model assumes CW signals and omni-directional projectors and receivers. We observed a good fit between model and experimental results for the long pulse data when the specular point was in the main lobe of both projector and receiver. The discrepancies observed in the other cases were attributed to beam pattern and pulse length effects. We have extended the model to incorporate these two effects. [Work supported by ONR Code 321US.]

\section{0:00-10:15 Break}

\section{0:15}

2aUW10. Measurements of mid-frequency bottom backscattering strengths on the Outer Hebrides Platform and the Stanton Banks. Edward L. Kunz and Roger C. Gauss (Nav. Res. Lab, Code 7140, Washington, DC 20375-5350, edward.kunz@nrl.navy.mil)

In July 2002, direct-path, mid-frequency $(2-5 \mathrm{kHz})$ bottom backscattering strength measurements were conducted at 21 sites on the Outer Hebrides Platform and the Stanton Banks (northwest of Scotland) during The Technical Cooperation Program's T-MAST 02 sea trial. This is a complex area, with bottom grabs indicating the wide presence of stones, pebbles, gravel and rocks throughout most of the region. When stones, etc. were present, bottom backscattering strengths were generally high and flattish in angle (over 10 to $40 \mathrm{deg}$ ), with many sites empirically exhibiting a $\sin \theta$ or flatter angular dependence. The frequency dependence in these cases was mild. When stones, etc. were absent, bottom backscattering strengths were less high and less flat in angle, with strong frequency dependence. These results dramatically illustrate the inadequacies of using Lambert's Law to model bottom backscattering strengths and suggest that active sonars operating in this environment will encounter strong reverberation. [Work supported by ONR.]

\section{0:30}

2aUW11. A semi-empirical model for predicting surface scattering strengths. Roger C. Gauss, Joseph M. Fialkowski, and Daniel Wurmser (Nav. Res. Lab, Code 7144, Washington, DC 20375-5350, roger.gauss@nrl.navy.mil)

Accurate monostatic and bistatic active sonar performance predictions require accurate predictions of reverberation which in turn rely on accurate estimates of surface scattering strength. This paper presents a new model that estimates the surface scattering strength due to both the rough air-sea interface (small-slope theory) and subsurface bubble clouds (a Lloydmirror model), given the acoustic frequency $(<5 \mathrm{kHz})$, incident grazing angle, scattered grazing angle, bistatic angle, wind speed, and sound speed in the water. This semi-empirical surface scattering strength (SESSS) model advances previous incarnations [Gauss and Fialkowski, J. Acoust. Soc. Am. 105, 1254 (1999) and ECUA 2000] by: (1) incorporating a low wavenumber cut-off to the surface-wave roughness spectra; (2) adding significant sets of low- and mid-frequency, open-ocean backscattering strength data to the database used to derive model parameters (using more comprehensive procedures); and (3) adding a new angle-dependent factor to the bubble-cloud scattering formula. All these changes have increased prediction accuracy, with variances of least-square fits to the new, full data set down from $9.7 \mathrm{~dB}^{2}$ for the old model to $5.3 \mathrm{~dB}^{2}$ for the new model. [Work supported by ONR and SPAWAR PMW-180.]

\section{0:45}

2aUW12. Backscattering of short acoustic pulses from 3-D rough surfaces: Statistical properties of first arrivals. Oleg A. Godin (CIRES, Univ. of Colorado and NOAA/Environ. Technol. Lab., 325 Broadway, Boulder, CO 80305), Iosif M. Fuks, and Mikhail I. Charnotskii (Zel Technologies, Broadway, Boulder, CO 80305)

Time history of a pulse backscattered by a rough surface contains information about position of the surface and properties of the scatterers. Such information is utilized successfully in a number of remote sensing techniques ranging from echo sounding of the ocean bottom to medical ultrasonics and satellite altimetry. In this paper, statistical properties of backscattered waves are considered in the geometrical acoustics approximation. The probing pulse duration is assumed to be sufficiently short so that signals backscattered in a vicinity of individual specular points on a rough surface do not overlap in time. Theoretical results previously obtained in a 2-D problem [I. M. Fuks and O. A. Godin, Waves Random Media 14, 539-562 (2004)] are extended to sound scattering from 3-D rough surfaces by following an approach developed in stochastic geometry. Predictions of an asymptotic theory are verified against results of a numerical simulation. Travel times and intensities of the first and the second arrivals of the backscattered wave are quantified in terms of statistical moments of roughness. It is found that, as in 2-D case, the travel time and the intensity are strongly correlated; on average, the earlier a signal arrives, the smaller is its intensity.

\section{1:00}

2aUW13. Backscattering from targets residing in caustics resulting from ocean boundary interactions. Benjamin R. Dzikowicz (Naval Surface Warfare Ctr., Panama City, FL 32407) and Philip L. Marston (Washington State Univ., Pullman, WA 99164-2814)

Detection of targets by backscatter in shallow water can be enhanced by interactions with ocean boundaries. A laboratory experiment is performed where a spherical target passes through an Airy caustic formed by a curved surface. When the target resides in the insonified region of the caustic there are two sets of multi-path rays: two pairs reflecting once off the surface (either to or from the target), and three reflecting twice off the surface (to and from the target). When a target moves across the caustic the singly reflected rays merge, as do the doubly reflected. With a longer tone burst the rays in each set overlap and the backscatter is greatly enhanced as the target moves into the insonified region. For a point target the singly reflected backscatter scales as an Airy function [B. R. Dzikowicz and P. L. Marston, J. Acoust. Soc. Am. 116, 2751-2757 (2004)], and the doubly reflected as the square of an Airy function. For a finite target the doubly reflected backscatter unfolds into a hyperbolic umbilic function. The arguments of the Airy and Hyperbolic Umbilic functions are calculated using the relative echo times of transient pulses. [Work supported by ONR.]

\section{1:15}

2aUW14. Determining flat interface reflection coefficients using forward scattering from a rippled sediment interface. Kevin L. Williams (Appl. Phys. Lab., Univ. of Washington, 1013 NE 40th St., Seattle, WA 98105)

For sediment interfaces that are very rough on the scale of the acoustic wavelength (i.e., $k h$ greater than 1 where $k$ is $2 * \pi$ /wavelength and $h$ is the rms roughness of the water/sediment interface) it is possible to estimate what the reflection coefficient would be if the interface were flat. In order to do so, a large ensemble of forward scattering measurements are 
needed in order to reduce the statistical uncertainty of the estimated reflection coefficient. In addition to the statistical uncertainty there can be biases in the estimate (for some grazing angles) that must be taken into account. The above conclusions will be supported through the use of monte carlo simulations of scattering from a rippled interface. The simulations are carried out in the context of discriminating between alternative acoustic models of sand sediments. [Work supported by ONR.]

\section{1:30}

2aUW15. Bayesian inversion of frequency-averaged reflection data. Stan E. Dosso (School of Earth and Ocean Sci., Univ. of Victoria, Victoria, BC, Canada) and Charles W. Holland (Penn State Univ., State College, PA 16801)

This paper presents a nonlinear Bayesian inversion of high-resolution seabed reflection data to estimate visco-elastic parameters of the upper sediments. The inversion is applied to data from two sites in the Strait of Sicily. One site is characterized by low-velocity, silty-clay sediments, resulting in data with a well-defined angle of intromission. The second site is characterized by high-velocity clayey sand, resulting in a critical angle. The data were frequency averaged from $500-2000 \mathrm{~Hz}$ and inverted for visco-elastic parameters of a half-space seabed model. The likelihood function employed in the inversion is based on the assumption of independent, Gaussian-distributed data errors, with the standard deviation included as a nuisance parameter in the inversion. Statistical tests are applied to the data residuals a posteriori to validate these assumptions. Good results are obtained for sediment compressional-wave velocity, compressional attenuation, and density; shear parameters are less-well determined although low shear-wave velocities are indicated. The Bayesian analysis provides a quantitative comparison of inversion results for the two sites in terms of the resolution of specific geoacoustic parameters, and indicates that the geoacoustic information content is significantly higher for angleof-intromission data.

\section{$11: 45$}

2aUW16. Bayesian inversion of multi-frequency reflection data with strongly correlated errors for density gradients. Jan Dettmer, Stan E. Dosso (School of Earth and Ocean Sci., Univ. of Victoria, Victoria, BC, Canada), and Charles W. Holland (Penn State Univ., State College, PA 16801)

This paper develops a non-linear Bayesian inversion for multifrequency reflection-loss data with strongly correlated data errors to resolve density and sound-velocity gradients which are often observed in the uppermost sediment layer. Although data errors are usually assumed to be independent in geoacoustic inversion, in reality measured data often show strong error correlations. The inversion developed here is designed to take error correlations into account. A full data covariance matrix is estimated from initial residuals of non-uniformly sampled data. This covariance matrix is then used in the likelihood function of a fast Gibbs sampler to sample the posterior probability density and provide parameter estimates and credibility intervals. Rigorous statistical tests are applied to the resulting data residuals to illustrate the benefits of this error treatment. The approach is applied to reflectivity data collected at a site characterized by low-velocity, water-saturated sediments in the Strait of Sicily. Density and sound-speed gradients are clearly resolved by the reflectivity data and agree with core measurements from the experiment site within the credibility bounds. 


\section{Meeting of the Standards Committee Plenary Group}

to be held jointly with the

\section{ANSI-Accredited U.S. Technical Advisory Group (TAG) Meetings for: ISO/TC 43 Acoustics ISO/TC 43/SC 1 Noise and IEC/TC 29 Electroacoustics}

P. D. Schomer, Chair, U.S. Technical Advisory Group (TAG) for ISO/TC 43 Acoustics and ISO/TC 43/SC 1 Noise 2117 Robert Drive, Champaign, IL 61821

V. Nedzelnitsky, U.S. Technical Advisor (TA) for IEC/TC 29, Electroacoustics National Institute of Standards and Technology (NIST), Sound Building, Room A147, 100 Bureau Drive, Stop 8221, Gaithersburg, MD 20899-8221

The meeting of the Standards Committee Plenary Group will precede the meetings of the Accredited Standards Committees $\mathrm{S} 1, \mathrm{~S} 2, \mathrm{~S} 3$, and S12, which are scheduled to take place in the following sequence:

\section{ASC S1 Acoustics \\ ASC S12 Noise \\ ASC S2 Mechanical Vibration and Shock and TAGs to TC 108 ASC S3 Bioacoustics}

\section{May 2005 \\ 17 May 2005}

18 May 2005

18 May 2005
1:45 p.m. to 3:15 p.m. 3:30 p.m. to 5:00 p.m.

8:00 a.m. to 9:30 a.m. 10:30 a.m. to 12:00 noon

Discussion at the Standards Committee Plenary Group meeting will consist of national items relevant to all S Committees.

The ANSI-Accredited US Technical Advisory Group (TAGs) for ISO/TC 43 Acoustics and IEC/TC 29 Electroacoustics, whose membership consists of members of $\mathrm{S} 1$ and $\mathrm{S} 3$, and other persons not necessarily members of these Committees, will meet during the Standards Plenary meeting. The ANSI-Accredited US Technical Advisory Group (TAG) for ISO/TC 43/SC 1 Noise, whose membership consists of the members of S12 and other persons not necessarily members of S12, will meet as well. The reports of the Chairs of these TAGs will not be presented at any other S Committee meeting. There will be a report on the interface of S1 and S3 activities with those of ISO/TC 43 and IEC/TC 29 including plans for future meetings of ISO/TC 43 and IEC/TC 29.

Members of S2 Mechanical Vibration and Shock (and U.S. TAG for ISO/TC 108 and its Subcommittees, (SC2, SC3, SC4, SC5, and SC6) are also encouraged to attend the Standards Committee Plenary Group meeting even though the S2 meeting will take place the next day.

The U.S. Technical Advisory Group (TAG) Chairs for the various international Technical Committees and Subcommittees under ISO and IEC, which are parallel to S1, S2, S3, and S12 are as follows:

\section{U.S. TAG Chair/Vice Chair \\ ISO \\ P. D. Schomer, Chair \\ P. D. Schomer, Chair \\ D. J. Evans, Chair \\ A. F. Kilcullen, Co-Chair \\ R. Taddeo, Co-Chair \\ D. J. Evans, Chair \\ D. D. Reynolds, Chair \\ D. J. Vendittis, Chair \\ R. F. Taddeo, Vice Chair \\ G. Booth, Chair}

IEC

V. Nedzelnitsky, U.S. TA
TC or SC

\section{U.S. TAG}

ISO/TC 43 Acoustics

$\mathrm{S} 1$ and $\mathrm{S} 3$

ISO/TC 43/SC1 Noise

S12

ISO/TC 108 Mechanical Vibration and Shock

S2

ISO/TC 108/SC2 Measurement and Evaluation of Mechanical Vibration and Shock as Applied to Machines, Vehicles and Structures

ISO/TC 108/SC3 Use and Calibration of Vibration and Shock Measuring Instruments

S2

ISO/TC 108/SC4 Human Exposure to Mechanical Vibration and Shock

S2

ISO/TC 108/SC5 Condition Monitoring and Diagnostics of Machines

S3

S2

ISO/TC 108/SC6 Vibration and Shock Generating Systems 


\title{
Session 2pAAa
}

\section{Architectural Acoustics, Education in Acoustics, Noise and Psychological and Physiological Acoustics: Topical Meeting on Classroom Acoustics-The Research Perspective III}

\author{
Murray R. Hodgson, Cochair \\ Univ. of British Columbia, School of Occupational and Environmental Hygiene, 2206 East Mall, \\ Vancouver, BC V6T 1Z3, Canada \\ Lily M. Wang, Cochair \\ Univ. of Nebraska Lincoln, Architectural Engineering, Peter Kiewit Institute, 1110 South 67th St., Omaha, NE 68182-0681
}

Chair's Introduction-1:10

Invited Papers

1:15

2pAAa1. Subjective evaluation of speech and noise in learning environments in the realm of classroom acoustics: Results from laboratory and field experiments. Markus Meis (Hearing Ctr. Oldenburg/Univ. of Oldenburg, Marie-Curie-Str. 2 , 26129 Oldenburg, Germany, markus.meis@ @oerzentrum-oldenburg.de), Christian Nocke (Akustikbuero Oldenburg), Simone Hofmann (Freudenberg Bldg. Systems), and Bernhard Becker (Deutsche Rockwool Mineralwoll GmbH \& CO. OHG)

The impact of different acoustical conditions in learning environments on noise annoyance and the evaluation of speech quality were tested in a series of three experiments. In Experiment $1(n=79)$ the auralization of seven classrooms with reverberation times from 0.55 to $3.21 \mathrm{~s}$ [average between $250 \mathrm{~Hz}$ to $2 \mathrm{kHz}$ ] served to develop a Semantic Differential, evaluating a simulated teacher's voice. Four factors were found: acoustical comfort, roughness, sharpness, and loudness. In Experiment 2, the effects of two classroom renovations were examined from a holistic perspective. The rooms were treated acoustically with acoustic ceilings (RT $=0.5 \mathrm{~s}[250$ $\mathrm{Hz}-2 \mathrm{kHz}]$ ) and muffling floor materials as well as non-acoustically with a new lighting system and color design. The results indicate that pupils $(n=61)$ in renovated classrooms judged the simulated voice more positively, were less annoyed from the noise in classrooms, and were more motivated to participate in the lessons. In Experiment 3 the sound environments from six different lecture rooms ( $\mathrm{RT}=0.8$ to $1.39 \mathrm{~s}[250 \mathrm{~Hz}-2 \mathrm{kHz}]$ ) in two Universities of Oldenburg were evaluated by 321 students during the lectures. Evidence found supports the assumption that acoustical comfort in rooms is dependent on frequency for rooms with higher reverberation times.

\section{$1: 45$}

2pAAa2. Prevalence, nature and risks of voice problems among public school teachers. Linda Rammage (Dept. of Surgery, UBC, PVCRP, 4th Fl. Willow Pavilion, VGH, 805 West 12th Ave, Vancouver, BC, Canada V5Z 1M9, lira@interchange.ubc.ca), Murray Hodgson (Univ. of British Columbia, Vancouver, BC V5Z 1M9), and Charlie Naylor (British Columbia Teachers Federation)

Voice problems among teachers represent a rising cause of teacher absenteeism, use of sick benefits, and stress among teachers and students. In British Columbia, the BC Teachers Federation and Workers Compensation Board are receiving increasing numbers of claims from teachers experiencing occupational voice problems and in the provincial voice clinic, the percentage of teachers in the clinic population is rising. Previous studies of teachers voice problems have typically had low return rates, which can bias the prevalence estimates, and have not incorporated standardized voice inventories, psychological inventories and acoustic measures. A survey study is in progress in B.C. to probe demographic, environmental, voice-use, health, psychological and personality issues that are thought to contribute to development of voice problems among teachers. To ensure validity of prevalence estimates by high return rates, on-site completion of questionnaires is being used in schools. Acoustical measures are also being made of representative classrooms, to determine the degree to which noise and reverberation contribute to voice problems among teachers.

\section{2:05}

2pAAa3. Benefits of teaching voice amplification as related to subjective laryngeal symptoms and perceived voice quality in teachers. Valdis Jonsdottir (Hraukbajarkot v/ Akureyri 601, Iceland)

Loud speaking due to noisy working conditions is a common cause for teachers' voice disorders. One way to diminish the vocal load of teaching is to make use of technical equipment. This Icelandic study explores: (1) if the use of amplification in classrooms would diminish the teachers' experienced symptoms of vocal fatigue; and (2) whether there is a possible change in perceptual voice quality during a teachers' working day. Thirty-three teachers, from grade school to university level, voluntarily served as subjects. They used amplifiers while teaching for one week at least. After that, they filled out a questionnaire concerning their symptoms and experiences. The results showed that the majority of teachers found amplification beneficial. They found it easier to talk and experienced less fatigue. The few disadvantages were technical. For a perceptual analysis, three females and two males (mean age 51 years) with long teaching experience and three or more dysphonic symptoms during the term, had their speech recorded while teaching, with and without amplification. In the clinical examination, no pathological changes were found in the vocal folds. In both studies, the quality of the voices was esteemed better when amplification was used. 
2pAAa4. Benefits of teaching voice amplification as related to subjective laryngeal symptoms in teachers, and to the listening conditions for pupils. Valdis Jonsdottir (Hraukbajarkot v/ Akureyri 601, Iceland)

Teachers unfavorable working conditions-large classrooms with poor acoustics, background noise and the great distance between teacher and pupils often are the main reasons for teachers voice problems. For pupils, the same circumstances simultaneously create unfavorable listening conditions. One way to diminish the vocal load of teaching is by making use of technical equipment. This Icelandic study explores whether voice amplification usage: (1) diminishes the teachers subjective symptoms of vocal fatigue; (2) makes it easier for pupils to listen and follow lessons; and (3) reveals other benefits and/or disadvantages of the usage of amplification in classrooms. Thirty-three teachers with their students (total 791), from grade school to university level, volunteered as subjects. The teachers used amplification in class for at least a week. At the end of the research period, the participants answered questionnaires, except the pupils between 6-9 years of age who were asked two questions. The results showed: (1) the majority of teachers found it easier to talk and experienced less fatigue using vocal amplification; (2) the students found it easier to hear the teacher through class chatter and to follow the lessons; (3) the disadvantages mentioned were mainly technical problems.

\section{2:45}

2pAAa5. Acoustic and other factors relating to the use of sound field systems in classrooms. Anne Carey, Bridget Shield (Dept. of Eng. Systems, Faculty of Eng., Sci. and Built Environment, London South Bank Univ., London SE1 OAA, UK), Julie Dockrell, and Kate Rigby (Univ. of London, 25 Woburn Square, London WC1H 0AA, UK)

A study has been made of thirty-five primary school classrooms which have Sound Field Systems (SFS) installed. Acoustic surveys of the classrooms have been undertaken and detailed observations made of the physical characteristics of each room such as its construction and design, plus any acoustic treatment. The positioning and type, e.g., column or separate speakers, of SFS installed were also noted. Details of the procedures for purchasing, installing, and maintaining the SFS were obtained where possible, together with information concerning training of teachers in their use. Results from these surveys suggest that in many cases SFS are installed in rooms where their performance may be compromised because of inadequate acoustic conditions. Additionally problems may arise due to inappropriate installation, choice of system, poor maintenance, or lack of training. The effectiveness of acoustic treatment such as absorption applied to ceilings and walls has also been investigated in a range of classrooms and its impact on the use of SFS considered.

\section{3:05-3:20 Break}

2pAAa6. Installation and impact of sound field systems on hearing and hearing impaired children and their teachers. Julie Dockrell, Kate Rigby (School of Psych. and Human Development, Inst. of Education, Univ. of London, 25 Woburn Square, London WC1H 0AA, UK), Bridget Shield, and Anne Carey (London South Bank Univ., London SE1 0AA, UK)

An evaluation of the installation and use of sound field systems in ten schools in England has been carried out. The evaluation included noise surveys of classrooms, questionnaire surveys of pupils and teachers and experimental testing of children with and without the use of SFS. The aim of this project was to investigate the impact of SFS on teaching and learning in elementary school classrooms, in particular, to ascertain whether the SFS differentially benefited children with hearing impairments. Barriers to teachers use of SFS were found in terms of equipment placement and maintenance, appropriate training, and teacher's knowledge. Nonetheless positive reports are recorded from both teachers and pupils. Teachers' and pupils' perceptions are compared with objective data evaluating change in performance when SFS are used for language and cognitive tasks. Data from children with hearing impairments and additional learning needs are analyzed for comparative purposes. The results are discussed in terms of effective practice for the use of SFS with elementary school pupils.

\section{$3: 40$}

2pAAa7. Acoustic and electromagnetic noise from lighting in classrooms. Charles A. Laszlo, Jonathan Lashin (UBC Dept. of Elec. and Comput. Eng., 2356 Main Mall, Vancouver, BC, Canada V6T 1Z4, claszlo@telus.net), and Murray R. Hodgson (UBC Acoust. and Noise Res. Group, Vancouver, BC, Canada V6T 1Z3)

Following complaints by hard-of-hearing students using assistive-listening devices, and their teachers, the hum-like noise generated by fluorescent lighting was investigated in classrooms and the school library in a typical school. This hum is caused by vibrations in the core of the magnetic ballasts. Measurements were made in several rooms without students present. Noise levels increased between 7 and $15 \mathrm{~dB}$ when fixtures using magnetic ballasts were switched on. Spectral analysis showed the presence of 30, 60, 120, and $240 \mathrm{~Hz}$ components. In rooms where electronic ballasts were installed, there was no increase in noise level when the lights were switched on. Since hearing aids and assistive-listening devices worn by students may also be influenced by magnetic fields, these were also surveyed in these classrooms. The magnetic fields generated by the lights were not significant, but near some wiring and electrical panels the interference was strong. In rooms with electronic ballasts some infrared assistive-listening devices picked up strong high-frequency hum. It is recommended that the effect of lighting fixtures and the electrical-distribution system be taken into account in the acoustical and communication design of classrooms. 
2pAAa8. Real world issues in classroom acoustics for hearing impaired students. Klaus Kleinschmidt (Acoust. Consultant, 132 Mary Catherine Dr., Lancaster, MA 01523)

Experience is being gained from evaluating and modifying the acoustical properties and background noise levels of existing classrooms for use by hearing impaired students. Projects include more than 25 schools in public school systems that are mainstreaming handicapped children. Various practical and economic restrictions have led to recommendations for modifications that do not necessarily comply with ANSI S12.60 2002. Examples of real world conditions and practical solutions will be described.
2pAAa9. Noise problem in a primary level classroom. Sergio Beristain (IMA, ESIME, IPN. P.O. Box 12-1022, Narvarte, 03020, Mexico D. F., Mexico. sberista@hotmail.com)

Noise was assesed in a primary school in Mexico City (six to twelve year old kids), known as having significant noise inside classrooms. Several sources were identified, among them, traffic noise and noise from the sports and recreational areas, which were in the center of the school with the classrooms surrounding them. Reverberation was an issue, but not very significant. But in some cases the most annoying sound (noise), was the one made by the students within the classroom, inducing a number of reactions in the lecturers. These reactions ranged from shouting to give the lecture, through trying to control the students, to ignore them and let it go. Lecturer's voice levels were also measured, finding out a wide spread in normal speech voice level, and some of their experiences and comments were analyzed. Results are sumarized in this presentation.

\begin{abstract}
Session 2pAAb
Architectural Acoustics and the National Council of Acoustical Consultants: Student Design Competition
\end{abstract}

Robert C. Coffeen, Cochair

Univ. of Kansas, School of Architectural and Urban Design, Marvin Hall, Lawrence, KS 66045

\author{
Byron Harrison, Cochair
}

The Talaske Group Inc., 105 N Oak Park Ave., Oak Park, IL 60301

The Technical Committee on Architectural Acoustics of the Acoustical Society of America and the National Council of Acoustical Consultants are sponsoring this Student Design Competition that will be professionally judged at this meeting. The purpose of this design competition is to encourage students enrolled in architecture, architectural engineering, and other university curriculums that involve building design and/or acoustics to express their knowledge of architectural acoustics in the design of a drama theater complex located within an urban mixed-use development. This competition is open to undergraduate and graduate students from all nations.

Submissions will be poster presentations that demonstrate room acoustics, noise control, and acoustic isolation techniques in building planning and room design.

The submitted designs will be displayed in this session and they will be judged by a panel of professional architects and acoustical consultants. An award of $\$ 1,250$ US will be made to the submitters of the entry judged "First Honors." Up to four awards of $\$ 700$ US each will be made to submitters of entries judged "Commendation." 


\title{
Session 2pAB
}

\section{Animal Bioacoustics: Methodology for Measurements of Auditory Evoked Potentials in Aquatic Mammals II}

\author{
Mardi C. Hastings, Chair \\ Office of Naval Research, ONR 341, 800 North Quincy St., Arlington, VA 22217
}

Chair's Introduction-1:25

Contributed Papers

$1: 30$

2pAB1. Effects of surveillance towed array sensor system (SURTASS) low frequency active sonar on fish. Arthur N. Popper, Michele B. Halvorsen, Diane Miller, Michael E. Smith, Jiakun Song, Lidia E. Wysocki (Dept. of Biol., Univ. of Maryland, College Park, MD 20742, apopper@umd.edu), Mardi C. Hastings (Office of Naval Res., Arlington, VA 22217), Andrew S. Kane (Univ. of Maryland, College Park, MD 20742), and Peter Stein (Sci. Solutions, Inc., Nashua, NH 03049)

We investigated the effects of exposure to Low Frequency Active (LFA) sonar on rainbow trout (a hearing non-specialist related to several endangered salmonids) and channel catfish (a hearing specialist), using an element of the standard SURTASS LFA source array. We measured hearing sensitivity using auditory brainstem response, effects on inner ear structure using scanning electron microscopy, effects on non-auditory tissues using general pathology and histopathology, and behavioral effects with video monitoring. Exposure to $193 \mathrm{~dB}$ re 1 microPa (rms received level) in the LFA frequency band for 324 seconds resulted in a TTS of 20 $\mathrm{dB}$ at $400 \mathrm{~Hz}$ in rainbow trout, with less TTS at 100 and $200 \mathrm{~Hz}$. TTS in catfish ranged from 6 to $12 \mathrm{~dB}$ at frequencies from 200 to $1000 \mathrm{~Hz}$. Both species recovered from hearing loss in several days. Inner ears sensory tissues appeared unaffected by acoustic exposure. Gross pathology indicated no damage to non-auditory tissues, including the swim bladder. Both species showed consistent startle responses at sound onsets and changed their position relative to the sound source during exposures. There was no fish death attributable to sound exposure even up to four days postexposure. [Work supported by Chief of Naval Operations.]

\section{$1: 45$}

2pAB2. The effects of stimulus parameters on the auditory brainstem response of zebrafish (Danio rerio). Maria Margaritis and Dennis Higgs (Dept. of Biol., Univ. of Windsor, Windsor, ON, Canada N9B 3P4, dhiggs@uwindsor.ca)

In mammals and birds it is well documented that stimulus parameters have significant effects on auditory processing. However in fish, the effect of different stimulus types remains unclear. Therefore it is difficult to directly compare piscine auditory responses obtained by different investigators, due to variability in testing methods. The current study uses zebrafish (Danio rerio) to evaluate the effects of stimulus type $(800 \mathrm{~Hz}$ tone bursts versus broadband stimuli from $100-4000 \mathrm{~Hz}$ ) and stimulus duration $(1,5,10$, or $20 \mathrm{~ms})$ on the auditory brainstem response (ABR). Threshold was significantly lower $(p=0.04)$ for broadband sounds than for tones, but there was no effect on either latency or amplitude. There was a direct and significant effect of duration on threshold $(p=0.001)$, suggesting the response was due to the offset rather than the onset of the stimulus. Duration had no effect on either threshold or amplitude. The stimulus level had no significant effect on latency, however amplitude significantly increased as level increased $(p=0.001)$. Thus, stimulus parameters can significantly affect ABRs. The current findings show the need for a consistent method of testing among investigators in order to reliably compare results. [Work supported by NSERC.]
2:00

2pAB3. Auditory evoked potential measurements in elasmobranchs. Brandon Casper and David Mann (Univ. of South Florida, College of Marine Sci., 140 Seventh Ave. South, St. Petersburg, FL 33701)

Auditory evoked potentials (AEP) were first used to examine hearing in elasmobranchs by Corwin and Bullock in the late 1970s and early 1980s, marking the first time AEPs had been measured in fishes. Results of these experiments identified the regions of the ear and brain in which sound is processed, though no actual hearing thresholds were measured. Those initial experiments provided the ground work for future AEP experiments to measure fish hearing abilities in a manner that is much faster and more convenient than classical conditioning. Data will be presented on recent experiments in which AEPs were used to measure the hearing thresholds of two species of elasmobranchs: the nurse shark, Ginglymostoma cirratum, and the yellow stingray, Urobatis jamaicencis. Audiograms were analyzed and compared to previously published audiograms obtained using classical conditioning with results indicating that hearing thresholds were similar for the two methods. These data suggest that AEP testing is a viable option when measuring hearing in elasmobranchs and can increase the speed in which future hearing measurements can be obtained.

2:15

2pAB4. Bottlenose dolphins audiogram dependence on azimuth: Evoked potential study. Vladimir Popov (Inst. of Ecology and Evolution, 33 Leninsky Prosp., 119071 Moscow, Russia)

ABR thresholds to tonal pips were measured in two bottlenose dolphins at different azimuthal positions of the sound source. The tested frequency range was from 8 to $128 \mathrm{kHz}$. Azimuth varied within a limit of 90 degree relative to the animals' longitudinal axis. This experimental paradigm allowed us to obtain ABR audiograms at different locations of the sound source. The zero-azimuth audiogram, at the sound source position in front of the animal, was of a standard appearance (minimum thresholds at frequencies of $3890 \mathrm{kHz}$, steep threshold increase at higher frequencies, and shallower increase at lower frequencies). The audiograms at lateralized sound-source positions looked in a different manner. With the azimuth increase, high-frequency thresholds rose much higher than low-frequency ones, so at azimuths of 6090 , the threshold versus frequency function was almost monotonous: the lowest threshold was observed at the lowest frequency $(8 \mathrm{kHz})$ and the highest threshold at the highest frequency $(128 \mathrm{kHz})$. With monaural ABR recording, audiograms contralateral to the sound source featured higher thresholds and steeper threshold increase with frequency as compared to the ipsilateral ones. [Work supported by the Russian Foundation for Basic Research.] 
rate transfer functions (MRTFs) were measured to establish how well the auditory system follows the temporal envelope of acoustic stimuli. A 40 $\mathrm{kHz}$ stimulus carrier was amplitude modulated (AM) with varying rates ranging from $200 \mathrm{~Hz}$ to $1800 \mathrm{~Hz}$, in $200 \mathrm{~Hz}$ steps. The best AM-rate from the first dolphin tested was $1500 \mathrm{~Hz}$. This AM rate was used in subsequent AEP measurements to determine evoked-potential hearing thresholds between 5000 and $80000 \mathrm{~Hz}$. These findings show that rough-toothed dolphins can detect sounds between 5 and $80 \mathrm{kHz}$, and are most likely capable of detecting frequencies much higher than $80 \mathrm{kHz}$. MRTF data suggest that rough-toothed dolphins have a high temporal resolution, similar to that of other cetaceans.

\section{3:00}

2pAB7. Stimulus-response characteristics of a harbor porpoise during active echolocation and passive hearing studied with auditory brain stem recordings (ABR). Kristian Beedholm and Lee A. Miller (Inst. of Biol., Univ. of Southern Denmark, Campusvej 55, DK-5230 Odense M, Denmark, lee@biology.sdu..dk)

We evaluated the stimulus-response characteristics of hearing by a harbor porpoise using narrow band pulses resembling the animal's own echolocation pulse $(130 \mathrm{kHz}, 100 \mathrm{us})$, but shifted in frequency $(80,100$, $125,160 \mathrm{kHz}$ ). Our animal was trained to accept two suction cup electrodes and to station $1 \mathrm{~m}$ below the water surface. Stimuli could be presented either as simulated echoes at a fixed delay $(5 \mathrm{~ms})$ relative to the animal's echolocation signals, or at a constant rate chosen by the experimenter. Stimulus levels were varied between 90 and $150 \mathrm{~dB}$ re $1 \mathrm{uPa}$ and the ABR responses were averaged (16 or more responses) at each level. The relationship between input level (in $\mathrm{dB}$ ) and ABR amplitude was reasonably linear for simulated echo and constant rate experiments. Regression lines were calculated to determine the level at which the response met the noise, defining the ABR threshold. There was little difference in the ABR threshold (100 to $110 \mathrm{~dB}$ re $1 \mathrm{uPa}$ ) for the four frequencies. The rate of growth of the $\mathrm{ABR}$ response with increasing stimulus level was steepest at $125 \mathrm{kHz}$, which could well reflect a relatively denser population of neurons tuned to this frequency area. [Work done at Fjord \& Baelt, Kerteminde, Denmark, and supported by ONR.] ported to Mote Marine Laboratory for rehabilitation. Two auditory evoked potential (AEP) measurements were performed on each of five of these dolphins in air using a jawphone to present acoustic stimuli. Modulation

\author{
Michael J. Buckingham, Chair \\ Scripps Inst. of Oceanography, 8820 Shellback Way, La Jolla, CA 92093-0238
}

Contributed Papers

1:30

2pA01. Seafloor classification using artificial neural network architecture from central western continental shelf of India. Vasudev Mahale, Bishwajit Chakraborty, Gajanan S. Navelkar, and R. G. Prabhu Desai (Natl. Inst. of Oceanogr., Dona Paula, Goa: 403 004, India, bishwajt@darya.nio.org)

Seafloor classification studies are carried out at the central western continental shelf of India employing two frequency normal incidence single beam echo-sounder backscatter data. Echo waveform data from different seafloor sediment areas are utilized for present study. Three arti- ficial neural network (ANN) architectures, e.g., Self-Organization Feature Maps (SOFM), Multi-Layer Perceptron (MLP), and Learning Vector Quantization (LVQ) are applied for seafloor classifications. In case of MLP, features are extracted from the received echo signal, on the basis of which, classification is carried out. In the case of the SOFM, a simple moving average echo waveform pre-processing technique is found to yield excellent classification results. Finally, LVQ, which is known as ANN of hybrid architecture is found to be the efficient seafloor classifier especially from the point of view of the real-time application. The simultaneously acquired sediment sample, multi-beam bathymetry and side scan sonar and echo waveform based seafloor classifications results are indicative of the 
depositional (inner shelf), non-depositional or erosion (outer shelf) environment and combination of both in the transition zone. [Work supported by DIT.]

\section{$1: 45$}

2pAO2. Geoacoustic parameters inversion by ambient noise in South China Sea experiment of ASIAEX. Ruey-Chang Wei, Yao-Hsien Kuo, Cheng-Hung Chen (Natl. Sun Yat-Sen Univ., No. 70, Lien-Hai Rd., Kaohsiung, Taiwan 804), and Hsiang-Chih Chan (Natl. Taiwan Univ., Taipei, 106, Taiwan)

Knowing bottom geoacoustic parameters is of great importance for using sonar systems effectively in shallow water. This study is based upon the vertical line array (VLA) in South China Sea experiment of the Asian Seas International Acoustic Experiment (ASIAEX), dated from May 3, 2001 to May 16, 2001, in which the ambient noise was also measured in shallow water. Beamforming is used to calculate the vertical directionality of ambient noise, but limited by the array element spacing, the major analysis frequency range is 100 to $400 \mathrm{~Hz}$. Comparison between forward modeling (normal mode code) and experimental results show the energy ratio of up-looking and down-looking beams, which can be referred to the energy reflection coefficient of the bottom-potentially a function of angle and frequency. From the reflection coefficient, critical parameters of the sediments such as sound speed, density, and attenuation coefficient can be obtained. Finally, this result is verified by on-site chirp sonar surveys during the experiment, and also by other inversion investigation in this area using other sound source.

\section{2:00}

2pAO3. Single beam echosounding: Considerations of depth and seabed slope. Ben R. Biffard, N. Ross Chapman, Steve F. Bloomer (School of Earth and Ocean Sci., Univ. of Victoria, P.O. Box 3055, Victoria, BC, Canada V8W 3P6), and Jon M. Preston (Quester Tangent Corp., Sidney, BC, Canada V8L 5Y8)

The topic of depth compensation of single beam echo time series for seabed classification is fairly well studied. The effect of seabed slope has not been publicized. In applications for seabed classification, seabed slope is observed to be associated with classification inaccuracy and failure. In cases of higher slope, single beam bathymetry also becomes inaccurate. A survey of 2 fjords with extreme variation in slope is presented as a representative example and testing bed for investigating slope. The direct effect on seabed echoes is investigated and explained in reference to a simple model of beam-echo geometry. Survey bathymetry is compensated for slope; inaccuracies of up to $5 \%$ of depth are corrected, however bottom picking accuracy is diminished in areas of slope and cannot be improved. Surveys of a gas hydrate site and a river will also be presented as applications of these ideas. Early results from BORIS model studies of methods for compensation of slope and depth may also be presented.

\section{2:15}

2pA04. Estimating the uncertainty of geoacoustic parameters of a range-dependent environment. Mark Fallat (Dept. of Earth and Ocean Sci., Univ. of BC, Canada), Stan Dosso (Univ. of Victoria, Canada), and Peter Nielsen (NATO Undersea Res. Ctr.)

In this study, data from a range-dependent environment are inverted to obtain estimates of the geoacoustic properties and their uncertainties. The technique consists of combining the results of a series of rangeindependent inversions to produce a model of the range-dependent environment. A rigorous uncertainty analysis provides a way of discerning whether variations in the inversion results are due to range-dependent features of the environment or simply due to uncertainty/variability in the results. Broadband acoustic data from a track off the island of Sicily were analyzed. Ground-truth information in the form of core measurements and a high-resolution seismic profile were also collected and used for verification of the results. The method of fast Gibbs sampling (FGS) was used to estimate the uncertainties of the geoacoustic properties. FGS is based on a Bayesian approach to inversion which samples the posterior probability distribution to estimate marginal probability distributions and parameter correlations. Marginal probability distributions were computed at various points along the track and compared to the ground-truth information. Overall, the analysis showed that the dominant range-dependent features of the environment could be estimated.

2:30

2pA05. A method for obtaining the geoacoustic properties of marine sediments using sound generated by aircraft. Eric M. Giddens and Michael J. Buckingham (Scripps Inst. of Oceanogr., La Jolla, CA 92093-0238)

A low-frequency geoacoustic inversion method has been developed for an isovelocity waveguide using light-aircraft as a source of sound. The high Doppler shift of the acoustic field facilitates the inversion, allowing directional information to be gathered by a single, omnidirectional receiver. The inversion method has been applied to simulations and to field experiments conducted in shallow water (14.4 m depth) off the coast of $\mathrm{La}$ Jolla. The inversion results will be discussed along with an error analysis using the Cramer-Rao Lower Bounds. [Work supported by ONR and the ARCS Foundation.]

\section{2:45}

2pAO6. Uncertainty bounds for geoacoustic inversions due to sound speed variations in water column. Ying-Tsong Lin and James F. Lynch (Woods Hole Oceanogr. Inst., Woods Hole, MA 02543)

The uncertainty bounds for geoacoustic inversion results due to sound speed variability in the water column perturbed by internal waves are assessed. Our previous work showed that if the sound speed variations are not taken account into the water column model, they will affect the accuracy of geoacoustic inversions. In this paper, we seek to quantify how well the error bounds could be given under certain knowledge of the sound speed variations. In doing so, the equivalent transform method derived from the linear perturbation theory plays a role in error transferral from water column to bottom. The water column variability is decomposed into ocean dynamic modes or as empirical orthogonal function (EOF) modes. Obtaining the ocean dynamic modes requires having the buoyancy frequency profile, whereas obtaining the EOF modes requires having vertical time series data. In general, it is difficult to assemble full statistics of the sound speed variations, so we examine the uncertainty bounds of the inversion results in the case where only partial statistics can be observed.

\section{3:00}

2pA07. Geoacoustic inversion of broadband data by matched beam processing. N. Ross Chapman and Yongmin Jiang (School of Earth and Ocean Sci., Univ. of Victoria, P.O. Box 3055, Victoria, BC, Canada V8W 3P6)

This paper describes results of geoacoustic inversion using broadband signals from an experiment carried out at a site near the South Florida Ocean Measurement Centre in the Florida Straits. M-sequence coded pulse trains at different center carrier frequencies from 100 to $3200 \mathrm{~Hz}$ were transmitted in the acoustic experiment, and received by a tri-axial array at a distance around $10 \mathrm{~km}$. Geoacoustic inversion was carried out to determine the possibility of inverting the environmental parameters from this long-range propagation experiment. The received signal at $100 \mathrm{~Hz}$ consisted of a dominant water column signal and a secondary arrival delayed by $0.4 \mathrm{~s}$. The secondary signal was spatially filtered by beamforming the array data, and the beam data were inverted by matched beam processing combined with an adaptive simplex simulated annealing algorithm. The estimated values of compressional wave speed and density were in good agreement with ground truth values from sediment cores. The inverted shear wave speed appears to be a sensitive parameter and consistent with compressional wave speed. Range and water depth were also included as inversion parameters, and the inversion results were close to the known values within small uncertainties. [Work supported by ONR.] 
and Reference Manual," Department of Ocean Engineering, Massachusetts Institute of Technology, 1997.] The PPDs resulting from the lab data are compared with those obtained for simulated data, and the effects of experimental error on the results are explored.

\section{$4: 15$}

2pAO11. Measurement of the bulk density gradient in the transition layer of a sand/water interface using a diver deployed X-Ray Attenuation Measurement System (XRAM). Todd Hay and Marcia Isakson (Appl. Res. Labs., Univ. of Texas, Austin, TX 78713)

The reflection coefficient is an important parameter for modeling acoustic applications in littoral environments. However, a density gradient in the transition layer of the interface can change the value of the reflection coefficient by $15 \mathrm{~dB}$ for high frequencies. Therefore, an accurate measurement of the bulk density gradient is crucial to the interpretation of reflection coefficient data. Previously, bulk density gradients have been determined by coring which disrupts grain microstructure or by electrical methods which do not measure the density directly. For this study, a novel means of directly determining the density gradient in situ using $\mathrm{x}$-ray attenuation was developed. The system is small, lightweight, and diver deployed allowing a greater flexibility in density measurements. Calibration measurements of materials with varying densities as well as measurements of the transition layer of a sand/water interface will be presented. [Work supported by ONR, Ocean Acoustics.]

\section{4:30}

2pAO12. Geoacoustic inversion for single paths of Haro Strait data. A. Tolstoy (A. Tolstoy Sci., 1350 Beverly Rd., Ste. 115 PMB 293, McLean, VA 22101)

This presentation will discuss efforts to date for the geoacoustic inversion of selected, single, Haro Strait Source-Receiver (SR) paths. The parameters emphasized will include water depth, linear sound-speed profiles, and the range between SR. The path will assume 3 segments: one segment for the region at $\mathrm{S}$, one for the region at $\mathrm{R}$, and one for an average region in between. The inversions will operate using the SUB-RIGS method which is based on multiple frequencies where higher frequencies are most sensitive to surficial geoacoustic properties while the lower frequencies are most sensitive to the deeper properties. Some paths have already been successfully inverted by earlier research efforts (Chapman et al.). This paper will demonstrate efforts on some of those paths as well as on other, more problematic SR paths.

\section{$4: 45$}

2pAO13. Classification of acoustic signals using the statistics of the 1-D wavelet transform coefficients. Michael I. Taroudakis (Dept. of Mathematics, Univ. of Crete and FORTH/IACM, P.O. Box 1527, 71110 Heraklion, Greece) and George Tzagkarakis (Univ. of Crete and FORTH/ ICS, Heraklion, Greece)

The representation of an acoustic signal in terms of the wavelet subband coefficients is studied using their statistical features. This type of representation is suggested as an alternative tool for tomographic or geoacoustic inversions. Using a set of representative shallow water environments, the variation of the statistical behavior of the sub-band coefficients is associated with corresponding variations of the environmental parameters. Low frequency signals suitable for ocean acoustic tomography are simulated. It is shown that the statistics of the sub-band coefficients are best described using non-Gaussian heavy-tailed distributions such as those of the alpha-stable family. The variations of the distribution parameters are measured using special statistical similarity functions such as the Kullback-Leibler divergence which has been adopted in the present study. The distances between the statistical parameters of a given and reference signals determined by these functions, can be used for subsequent nonlinear inversions aiming at the recovery of the environmental parameters. 


\title{
Session 2pBB
}

\section{Biomedical Ultrasound/Bioresponse to Vibrational and Physical Acoustics: Ultrasound Imaging}

\author{
Jeffrey A. Ketterling, Cochair \\ Riverside Research Inst., 156 William St., New York, NY 10038-2609 \\ James C. Lacefield, Cochair \\ Dept. of Electrical and Computer Engineering, Univ. of Western Ontario, London, ON N6A 5B9, Canada
}

Chair's Introduction-1:45

\section{Contributed Papers}

\section{$1: 45$}

2pBB1. Computational synthesis of ultrasound breast images from a three-dimensional anatomical model. Yi-Ting Shen and James C. Lacefield (Univ. of Western Ontario and Robarts Res. Inst., London, ON, Canada)

A three-dimensional breast anatomy model has been implemented using spline surfaces and fractal structures to represent the architecture of the lactiferous ducts, mammary fat lobules, skin, and supporting connective tissues. The model randomly varies user-specified structural parameters to provide an unlimited number of realizations of the gross anatomy. Cross-sectional views extracted by slicing through a realization of the model are input to a two-dimensional $k$-space (i.e., spatial frequency domain) ultrasound propagation simulator. The $k$-space simulator iterates pressure and particle velocity fields in 30-ns steps to compute scattering from the structures defined by the anatomical model and small random variations in compressibility that are added to generate speckle. A synthetic aperture method is employed to simulate $B$-mode imaging with a 5 $\mathrm{MHz}, 192$-element linear array operated using multiple transmit focal zones and dynamic receive focusing. Simulated images of randomscattering phantoms possess approximately Rayleigh speckle statistics. The anatomical model is expected to yield images with speckle statistics comparable to clinical breast images. The long-term objectives of these simulations are to investigate sources of focus aberration in ultrasound breast imaging and the impact of aberration on cancer detection. [Work supported by an NSERC Discovery Grant.]

\section{2:00}

2pBB2. Tissue-type imaging (TTI) based on ultrasonic spectral and clinical parameters for detecting, evaluating, and managing prostate cancer. Ernest J. Feleppa, Jeffrey A. Ketterling, Shreedevi Dasgupta, Andrew Kalisz, Sarayu Ramachandran (Biomed. Eng. Labs., Riverside Res. Inst., 156 William St., New York, NY 10038, feleppa@rrinyc.org), and Christopher R. Porter (Virginia Mason Medical Ctr., Seattle, WA 98101)

This study seeks to develop more-sensitive and -specific ultrasonic methods of imaging cancerous prostate tissue and thereby to improve means of guiding biopsies and planning, targeting, and monitoring treatment. Ultrasonic radio-frequency, echo-signal data, and clinical variables, e.g., PSA, voiding function, etc., during biopsy examinations were acquired. Spectra of the radio-frequency signals were computed in each biopsied region, and used to train neural networks; biopsy results served as the gold standard. A lookup table gave scores for cancer likelihood on a pixel-by-pixel basis from locally computed spectral-parameter and global clinical-parameter values. ROC curves used leave-one-patient- and leaveone-biopsy-out approaches to minimize classification bias. Resulting ROC-curve areas were $0.80 \pm 0.03$ for neural-networks versus $0.66 \pm 0.03$ for conventional classification. TTIs generated from data acquired presurgically showed tumors that were unrecognized in conventional images and during surgery. 3-D renderings of prostatectomy histology and TTIs showed encouraging correlations, which shows promise for improving the detection and management of prostate cancer, e.g., for biopsy guidance, planning dose-escalation and tissue-sparing options for radiation or cryotherapy, and assessing the effects of treatment. Combining MRS parameters with US spectral parameters appears capable of further improving prostate-cancer imaging. [Work supported by NIH.]

\section{2:15}

2pBB3. Bioheat Transfer Model (BHTE) based temperature estimation technique for high intensity focused ultrasound therapy monitoring. Peter J. Kaczkowski and Ajay Anand (Appl. Phys. Lab, Univ. of Washington, 1013 NE 40th St., Seattle, WA 98105)

The spatial distribution of tissue temperature is an essential indicator of thermal therapy progress, treatment safety and efficacy. Here, it is shown through in vitro experiments that temperature rise can be accurately measured during therapy delivery and post-treatment cool down phases using RF backscatter data collected with a modified ultrasound scanner. $\mathrm{RF}$ data is acquired prior to, during, and after HIFU exposures, in tissue mimicking phantoms and excised animal tissue. Through two calibration experiments, initial estimates for key BHTE parameters (local thermal diffusivity, and magnitude of the HIFU heat source) and the temperature dependence of ultrasonic travel time are obtained prior to therapy. Tissue heterogeneity over a larger targeted region of interest is modeled as a change in the magnitude of the focal heat source. During therapy this magnitude is updated using an iterative optimization technique that minimizes the difference between predicted and measured travel time values. Temperature and thermal dose maps are generated throughout therapy delivery and post-treatment cooling periods. The ultrasound derived estimates are validated against independent thermocouple measurements close to but not at the HIFU focus. This model-based technique permits noninvasive temperature estimation throughout the entire therapeutic range, and is thus a departure from previously reported techniques.

\section{2:30}

2pBB4. Comparison of three models at high frequency for ultrasound tissue scattering. Michael Oelze, William O’Brien, Jr. (Dept. of Elec. and Comput. Eng., Univ. of Illinois at Urbana-Champaign, Urbana, IL 61801), and James Zachary (Univ. of Illinois at Urbana-Champaign)

A mammary carcinoma was grown in mice and imaged with an ultrasound transducer operating with a center frequency of $65 \mathrm{MHz}$. Quantitative ultrasound (QUS) analysis was used to characterize the tumors using the bandwidth of 30 to $85 \mathrm{MHz}$. Three models (Gaussian scatterer, fluidfilled sphere, and a new cell scatterer) for scattering were examined and scatterer property estimates were compared to real tissue morphology as seen from optical microscope images of the tumors. The Gaussian scattering model did not fit the data well compared to the fluid-filled sphere and new cell scatterer models. The fluid-filled sphere model fit the measure- 
ments better than any other model but did not yield scatterer property estimates that resembled underlying structure. Using the fluid-filled sphere model, the average estimated scatterer diameter was $25.5 \pm 0.14 \mu \mathrm{m}$. A new cell scatterer model was developed, which was based on scattering from a cell by incorporating the effects of the cytoskeleton and nucleus. The new cell scatterer model yielded estimates that appeared to reflect underlying structure more accurately. Using the new cell scatterer model, the average estimated nuclear diameter was $15.6 \pm 2.2 \mu \mathrm{m}$ compared with $13.2 \mu \mathrm{m}$ as measured from optical microscope images.

\section{2:45}

2pBB5. Importance of local attenuation on the estimation of scatterer size from ultrasound backscattered waveforms. Timothy A. Bigelow and William D. O’Brien, Jr. (Univ. of Illinois, 405 N. Mathews Ave., Urbana, IL 61801, bigelow@uiuc.edu)

Estimating the characteristic size of the tissue microstructure from a statistical analysis of the backscattered power spectrum could improve the diagnostic capability of medical ultrasound. Previously, size estimates were obtained for focused sources after compensating for source focusing, the frequency-dependent attenuation along the propagation path (total attenuation), and the frequency-dependent attenuation in the region of interest (local attenuation) given the attenuation values. In this study, the importance of the local attenuation on the scatterer size estimate was determined using computer simulations. The simulations used Gaussian impedance distributions with an effective radius of $25 \mu \mathrm{m}$ randomly positioned in a homogeneous half-space (attenuation from 0.05 to $1 \mathrm{~dB} / \mathrm{cm}$ $\mathrm{MHz}$ ) sonified by a spherically focused source $(f / 1$ to $f / 4)$. The total attenuation and focusing were assumed known. The scatterer size was estimated assuming that the local attenuation was zero, the local attenuation was the mean value of the true attenuation over the frequency range used to obtain the estimates, and the local attenuation was $0.5 \mathrm{~dB} / \mathrm{cm}$ $\mathrm{MHz}$. These three size estimates were then compared to estimates obtained using the true local attenuation value as well as estimates obtained when both focusing and local attenuation were not compensated.

\section{3:00-3:15 Break}

\section{3:15}

2pBB6. Non-invasive measurement of in situ thermal diffusivity and local heat source using backscattered ultrasound for thermal therapy planning and monitoring. Ajay Anand and Peter Kaczkowski (Univ. of Washington, 1013 40th St NE, Seattle, WA 98105)

Bioheat transfer equation (BHTE) estimates of applied dose during HIFU therapy typically use prior knowledge or assume standard values for tissue properties that determine thermal diffusivity $(K)$ and heat source $(Q)$. We have developed a novel signal-processing based technique to noninvasively estimate these parameters in situ based on analysis of raw backscattered RF data from two localized HIFU exposures, one at subablative intensities to determine $K$, and another at therapeutic intensities to obtain $Q$. Both exposures are performed prior to therapy. To estimate $K$, a short HIFU exposure is applied resulting in a temperature rise of less than $15^{\circ} \mathrm{C}$. The Gaussian radius of the temperature induced apparent strain profile during cool down is estimated and fit to an approximate analytical heat diffusion expression to obtain $K$. Independent estimates of $K$ derived using the transient hotwire technique validated the ultrasonic measurements. To estimate $Q$, an audio range hydrophone was acoustically coupled to the sample to detect the onset of boiling. The time required to bring the sample to boiling was used to estimate $Q$ by iteration of a numerical BHTE model. These results are validated against in situ measured values using thermocouples and linear acoustic calculations.
3:30

2pBB7. Validation of high-frequency ultrasound measurements of tissue layer thickness. Qiang Qiu, Joy Dunmore-Buyze, Derek R. Boughner, and James C. Lacefield (Univ. of Western Ontario, Robarts Res. Inst., and London Health Sci. Ctr., London, ON, Canada)

High-frequency ultrasound imaging enables nondestructive measurement of layer thickness in tissue specimens. These measurements are valuable for mechanical testing of soft biomaterials. This paper demonstrates a method for assessing the accuracy of high-resolution ultrasonic thickness estimates. Three-dimensional images of six porcine aortic valve cusps were acquired in vitro using a $40 \mathrm{MHz}$ ultrasound system with $40 \times 80$ $\times 80 \mu \mathrm{m}^{3}$ spatial resolution. The cusps were then frozen in liquid nitrogen, sectioned into $10-\mu \mathrm{m}$ slices, and micrographs of one slice from each specimen were acquired at $4 \times$ magnification. The two-dimensional micrographs were registered to the three-dimensional ultrasound images using a cross-correlation method. The boundaries of the fibrosa, spongiosa, and ventricularis layers were segmented in both sets of images using an active contour model. The average thicknesses of the tissue layers in the registered images were estimated and the absolute differences of the optical and ultrasonic estimates were computed. The absolute differences were $55.8 \pm 22.6 \mu \mathrm{m}$ (mean \pm standard deviation), 23.5 $\pm 14.3 \mu \mathrm{m}$, and 22.7 $\pm 17.2 \mu \mathrm{m}$ for the fibrosa, spongiosa, and ventricularis, respectively. The measurement differences are comparable to the axial resolution of the ultrasound system and are not significant as determined by $t$-tests ( $p$ $>0.30$ for each layer).

\section{3:45}

2pBB8. Automatic 3D acoustic tissue models from histologic tissue sections and application to ex vivo tissue characterization. Jonathan Mamou, Michael L. Oelze, William D. O'Brien, Jr. (Dept. of Elec. and Comput. Eng., Univ. of Illinois at Urbana-Champaign, 1406 W. Green St., Urbana, IL 61801, mamou@uiuc.edu), and James F. Zachary (Univ. of Illinois at Urbana-Champaign, Urbana, IL 61801)

Three-dimensional acoustic tissue models (3DATMs) can be used as computational tools for ultrasonic imaging algorithm development and analysis. 3DATMs are automatically constructed from digitized light microscope images of consecutive H\&E-stained histologic tissue sections. Construction necessitated contrast equalization, registration, and interpolation of missing sections. The registered (with interpolated) sections yield a $3 \mathrm{D}$ histologic volume (3DHV). Acoustic properties are then assigned to each tissue constituent of the 3DHV to obtain the 3DATM. A tissue characterization technique was developed to obtain scatterer parameter estimates (size and acoustic concentration) from a 3D impedance map (3DZM) deduced from a 3DHV by assigning acoustic impedance values. 3DZMs were constructed for a rat fibroadenoma (FA), a mouse mammary tumor (MMT) and a mouse sarcoma (EHS). From these 3 3DZMs estimates, effective scatterer diameters of $91 \mu \mathrm{m}, 31.5 \mu \mathrm{m}$, and $34.5 \mu \mathrm{m}$, respectively, were determined. Independent ultrasonic measurements yielded average scatterer diameters of $105 \mu \mathrm{m}, 30 \mu \mathrm{m}$, and $33 \mu \mathrm{m}$, respectively. The 3DZM estimation scheme showed results similar to those obtained by the ultrasonic measurements. 3DATMs may therefore be a useful tool for quantifying ultrasonic tissue properties. [Work supported by the University of Illinois Research Board.]

\section{4:00}

2pBB9. Automated set of test simulations for non-ideal medical ultrasound transducers using Monte-Carlo method. Hotaik Lee, Nadine B. Smith (Grad. Program in Acoust., The Penn State Univ., University Park, PA 16802), and Terry A. Kling (Sound Technol. Inc., State College, PA 16803)

Using Field II program and Monte-Carlo method, we developed new design tools for medical ultrasound transducers with the automated set of imaging simulations. This simulation environment is used to create and assess the parametric specification of design factors affecting the quality of medical ultrasound imaging. In order to obtain accurate and realistic results, non-ideal transducers whose transfer functions vary either across the transducer or within the transducer are considered. These variations 
include, but are not limited to: center frequency, bandwidth, sensitivity, ringdown, angular response, time-of-flight, and lateral focus. By applying random numbers within the tolerance range to the variations in input parameters, the automated set of simulations is performed. First, critical input parameters for components of the transfer function are identified by sensitivity analysis. Next, the statistical range of parameter values that yield a transducer model with a certain performance level is determined and the limit of variations in each factor for acceptable degradation of images is set. Finally, the creation of many "what if" cases to predict yield and statistical performance of a transducer and the imaging simulation are performed based on Monte-Carlo method. [Work supported by Sound Technology Inc.]

TUESDAY AFTERNOON, 17 MAY 2005

GEORGIA A, 1:00 TO 4:05 P.M.

\title{
Session 2pEA
}

\section{Engineering Acoustics, Acoustical Oceanography and Underwater Acoustics: Underwater Acoustic Sensor Technologies}

\author{
Dehua Huang, Cochair \\ Naval Undersea Warfare Center, 1176 Howell St., Newport, RI 02841-1708 \\ Thomas R. Howarth, Cochair \\ NAVSEA Newport, 1176 Howell St., Newport, RI 02841 \\ Chair's Introduction-1:00 \\ Invited Papers
}

1:05

2pEA1. Injection-molded 1-3 piezocomposite sensor development: The last ten years. Kim C. Benjamin (Naval Sea Systems Command Div. Newport, 1176 Howell St., Newport, RI 02841)

The past ten years have seen several interesting demonstrations of 1-3 piezocomposite when used as the active component in sonar sensors. Initially considered receive only by most in the field, piezocomposite has evolved into a proven broadband transducer material with both receive and transmit capability. From large aperture single element calibration transducers, to parametric mode projectors, the material has surprised many experts with its power handling capability. Its polymer constituent provides an amazing degree of versatility by allowing the thermoforming and shaping of transducer substrates for packaging into todays undersea vehicles. This talk will review the last ten years of piezocomposite transducer and array development focusing on both the materials transmit behavior and fabrication benefits for future sonar applications. [Work supported by the U.S. Navy.]

\section{1:30}

2pEA2. Engineering applications of limited diffraction beams. Jian-yu Lu (Ultrasound Lab, Dept. of Bioengineering, The Univ. of Toledo, Toledo, OH 43606, jilu@eng.utoledo.edu)

Limited diffraction beams (LDBs) are non-divergence and non-dispersive solutions to isotropic-homogeneous wave equations. These beams have a common characteristic of $\mathrm{X}$-shaped branches and thus are also called $\mathrm{X}$ waves. Because of their highly directional propagation property, they have potential applications in medicine, underwater acoustics, and nonlinear optics (Charles Day, Phys. Today, October, 2004, pp. 25-26). In this talk, an overview of the development of LDBs will be given. This includes the conversion of any existing solutions to homogeneous or non-homogeneous wave equations to LDB solutions using Lorentz-related transformation, and a discussion of the orthogonal properties of the $\mathrm{X}$ wave transformation pair in representing any physically realizable waves. Experiments on applications of LDBs using our newly developed general-purpose ultrasound system will also be reported. (The system is capable of 128-channel simultaneous ultrasound data acquisitions at 12 -bit/40 MHz rate and can hold real-time radiofrequency (RF) data up to $64 \mathrm{~GB}$ in one acquisition operation. Arbitrary ultrasound waveforms can be simultaneously produced by 128 12-bit/40 MHz D/A converters and then be linearly amplified to drive 75 -Ohm loads or an array transducer at about $\pm 150 \mathrm{~V}$.)

\section{$1: 55$}

2pEA3. Fiber optic acoustic sensor technology. James Cole, Clay Kirkendall, and Anthony Dandridge (Naval Res. Lab, 4555 Overlook Ave., SW, Washington, DC 20375)

Fiber optic sensor technology has been under development for over 25 years, recently a major milestone has been reached- the introduction of the Fiber Optic Wide Aperture Array on the first Virginia class submarine. This paper will review the development of this technology, outlining the principles of operation and the technological developments that led to fiber optic interferometric sensors becoming viable for production in an advanced sonar system. The Fiber Optic Wide Aperture array is a large channel count planar array mounted on the side of the submarine, but fiber sensor technology is also being developed for both towed arrays (as a replacement for the Navy's thin-line towed arrays) and for bottom mounted acoustic arrays for a number of Navy applications. This 
paper will describe the development of the fiber optic hydrophones for these applications as well as the optical interrogation techniques. One of the key features of fiber optic sensor technology is the ability to passively multiplex many hydrophone channels per fiber, several multiplexing techniques will also be described. Some of the issues and challenges of this technology, such as coherent noise for large channel count systems will also be briefly discussed.

\section{Contributed Papers}

\section{2:20}

2pEA4. Twenty years of barrel-stave flextensional transducer technology in Canada. Dennis F. Jones (Defence R\&D CanadaAtlantic, P.O. Box 1012, Dartmouth, NS, Canada B2Y 3Z7, dennis.jones@ drdc-rddc.gc.ca)

The barrel-stave flextensional transducer, a compact underwater sound source, was conceived at DRDC Atlantic in 1986 [G. W. McMahon and D. F. Jones, U.S. Patent No. 4,922,470 (1 May 1990); Canadian Patent No. $1,285,646$ (2 July 1991)]. Over the years, five barrel-stave designs belonging to three flextensional classes were built and tested at DRDC Atlantic. Three Class I transducers with operating frequencies ranging from 800 to $1600 \mathrm{~Hz}$ were integrated into submarine communications buoys, low frequency active horizontal projector arrays, and a broadband sonar towbody. A high-power Class II and broadband (1-7 kHz) Class III transducer were deployed under the ice in the Lincoln Sea for research related to rapidly deployable surveillance systems. These barrel-stave flextensional transducers have also supported a variety of marine mammal studies including vocal mimicry in long-finned pilot whales, coda dialects in sperm whales, and the R\&D of acoustic detection and tracking systems for endangered northern right whales. In August 2004 a barrel-stave transducer was used to lure a trapped juvenile humpback whale to the sluice gates of a tidal generating station on the Annapolis River in Nova Scotia by transmitting humpback whale calls underwater. The acoustic performance parameters for all 5 transducers will be presented.

\section{2:35-2:50 Break}

\section{2:50}

2pEA5. Single crystal cylinder transducers for sonar applications. Harold Robinson, Gerald Stevens, Martin Buffman (NUWC Div. Newport, 1176 Howell St., Newport, RI 02841), and James Powers (EDO Corp., Salt Lake City, UT 84115)

A segmented cylinder transducer constructed of single crystal lead magnesium niobate-lead titanate (PMN-PT) has been under development at NUWC and EDO Corporation for several years. The purpose of this development was to provide an extremely compact, high power broadband source. By virtue of their extraordinary material properties, ferroelectric single crystals are the ideal transduction material for developing such compact broadband systems. This presentation shall review the evolution of the transducer design as well as present the results of a successful in-water test conducted at NUWC in October of 2003. It shall be shown that design changes intended to eliminate spurious modes limiting the transducer bandwidth first observed in 2002 were successful, resulting in a transducer with a clean frequency response and an effective coupling factor of 0.85 . The measured transducer admittance was in nearly exact agreement with theoretical predictions. The NUWC in-water tests demonstrated that the single crystal cylinder achieved an admittance bandwidth (based on the Stansfield criterion) of over $100 \%$, while the tuned power factor was 0.8 or more over 2.5 octaves of frequency. Additionally, the transducer produced $12 \mathrm{~dB}$ higher source levels than a similarly sized PZT transducer. [Work sponsored by DARPA.]

\section{3:05}

2pEA6. Dipole projectors for conformal sonar system applications. Michael D. Gray (Acoust. and Mech. Systems Div., Georgia Tech Res. Inst., Atlanta, GA 30332-0810), Peter H. Rogers, and Gregg D. Larson (Georgia Inst. of Technol., Atlanta, GA 30332-0405)

Dipole projectors are being investigated for use in active sonar arrays on compliantly coated, low input impedance hulls. When the projector standoff $d$ is acoustically small $\left(k_{0} d<1\right)$, the direct and hull-reflected signals add in phase in the far field, leading to an increase in signal strength on the order of 2 relative to a free field dipole. By contrast, the direct and reflected signals for monopole transducer will have nearly opposite phase, and the monopole will have a reduction in total signal strength on the order of $2 k_{0} d$. Ideally, both transducer types have cosine directivities in-situ, although the monopole transducer response can be more strongly influenced by hull elastic contributions. Designs for two simple realizations of a dipole projector will be presented, along with beam pattern measurements made with prototype transducers at the Georgia Tech water tank facility.

$3: 20$

2pEA7. A novel underwater acoustic transmitter. Chung Chang and Richard Coates (Schlumberger-Doll Res., 36 Old Quarry Rd., Ridgefield, CT 06877)

A strong wide bandwidth low-frequency acoustic transmitter was designed and built to operate in the high-pressure environment of $20000 \mathrm{psi}$ and temperature over $200{ }^{\circ} \mathrm{C}$ inside an oil well. The same design idea can be easily adapted to the naval application in order to use it in the deep ocean environment. This transmitter is pressure balanced and does not use conventional piezoelectric material. Therefore, there is no performance degradation with changing pressure and temperature. This new transmitter uses impact force onto an acoustic resonator to generate sound. The impact force is generated by an electrical hammer. The design is simple, robust and the radiated acoustic wave fields are as repeatable as the piezoelectric source. In addition this new transmitter does not require an expensive amplifier to operate. A video clip will be shown to demonstrate the strength of the source. Further research was done to understand the impact physics and its controlling parameters. Using signal deconvolution technique one can measure the impact force function. A peak impact force of a few thousand pounds by the hammer was measured.

\section{$3: 35$}

2pEA8. 3D spatial sampling with a cylindrical multibeam sonar array. Daniel S. Brogan and Christian P. de Moustier (Ctr. for Coastal and Ocean Mapping, Univ. of New Hampshire, 24 Colovos Rd., Durham, NH 03824-3525, daniel.brogan@unh.edu)

Various beam pair combinations can be formed with cylindrical multibeam sonar arrays to obtain a 3D spatial sampling of a patch of seafloor for each ping. This capability is explored with a $286 \mathrm{deg}$ sector cylindrical array transmitting a stepped FM pulse over a 243 deg vertical fan beam centered on nadir and receiving with twenty-seven beam pairs, symmetrically steered about nadir in the fore-aft direction and spaced at $7.16 \mathrm{deg}$ intervals across track. Conventional conjugate product techniques yield two across-track profiles of 26 soundings each (52 phasors total) per ping. However, by combining one or two phasors along-track and one, two or three phasors across-track, soundings can be obtained at the spatial bisectors of the angles between the 52 phasors. This yields three profiles consisting of 51 soundings each, for a total of 153 soundings. This effectively creates a 3D patch of detected seafloor for each ping, which may overlap with the patches from adjacent pings depending on the sonars attitude changes and altitude above the seafloor. [Work supported by NRL-SSC grant N00173-00-1-G912 and NOAA grant NA170G228.] 
2pEA9. Techniques in piezoelectric transducer health monitoring. Eli M. Hughes I, Karl M. Reichard, and Tom B. Gabrielson (Penn State Appl. Res. Lab., 3075 Research Dr., CATO Park State College, PA 16801)

For many high intensity underwater applications, the piezoelectric elements within a transducer can be placed under high thermal, electrical and mechanical stress. One particular area of piezoelectric transducer research that has received little attention is in-situ health monitoring and failure prediction of the piezoelectric elements. While some studies have been performed in the theoretical mechanics of fractures due to device stress, there is a lack of practical information about piezoelectric health practical information about a transducer's electrical characteristics as it is stressed such that failure predictions can be made. The two major failure modes examined in this study were electro-mechanical stress and thermal depolarization. A large part of this research was devoted to the development of novel low-cost electronics that permit measurement of the broad band electrical impedance of the device from 0 to $50 \mathrm{kHz}$ in a small time aperture. The new measurement techniques allow for a large amount of visibility in how a transducer's electrical impedance changes before and during failure. This added visibility provides information that can be used to accurately predict if a transducer is failing and what mechanism is causing the failure.

TUESDAY AFTERNOON, 17 MAY 2005

REGENCY F, 1:00 TO 4:30 P.M.

\title{
Session 2pNS
}

\section{Noise: Environmental Noise and Noise Criteria}

\author{
John Erdreich, Chair \\ Ostergaard Acoustical Associates, 200 Executive Dr., West Orange, NJ 07052
}

\section{Contributed Papers}

\section{1:00}

2pNS1. Blast noise impacts on sleep. Edward T. Nykaza and Larry L. Pater (Eng. Res. and Development Ctr., Construction Eng. Res. Lab., 2902 Farber Dr., Champaign, IL 61822)

Firing large guns during the hours of darkness is essential to combat readiness for the military. At the same time most people are particularly sensitive to noise when sleeping or trying to fall asleep. Laboratory studies done by Griefahn [J. Sound and Vib. 128, 109-119 (1989)] and Luz [see Luz et al., ERDC/CERL, TR-04-26 (2004)] suggest that a time period at night may exist where people are more tolerant to large weapon impulse noise (blast noise) and therefore, are less likely to be awakened from noise events. In the fall of 2004, a field study was conducted around a military installation to determine if such a time period(s) exists. Noise monitors were set up inside and outside of residents homes to record noise levels from live military training activities and actimeters were worn by participants sleeping their natural environment to measure sleep disturbance and awakening. The method and results of this study will be presented. [Work supported by US Army Engineer Research and Development Center CERL.]

\section{1:15}

2pNS2. Development of metrics to identify military impulse noise. Jeffrey S. Vipperman (Dept. of Mech. Eng., Univ. of Pittsburgh, 648 Benedum Hall, Pittsburgh, PA 15261)

Urban encroachment of military bases continues to be a serious problem, affecting training exercises and overall military readiness. The military currently performs noise monitoring around bases to ensure that environmental conditions are favorable for tests or exercises. Despite refinements, current monitoring systems still suffer from occasional false positives and rely heavily on human interpretation. The long range goal of this work is to create a more accurate and autonomous noise classifier. The specific aims of the work are to create a library of recorded military impulse noise sources and to develop, test, and refine the noise classifier algorithms. Representative, high quality field measurements of various noise sources ( $25 \mathrm{~mm}$ and larger) will be conducted at a few military bases under varying environmental conditions. Measurements of wind noise and thunder will also be recorded. The software will be developed in MAT$\mathrm{LAB}$ and will employ several common impulse noise metrics used for hearing conservation, including rise, peak, and fall times, crest factor, $A$ - and $B$-durations, $L_{p k}$ and kurtosis, among others. A neural network will be trained to discern between the recorded military noise sources and naturally occuring noise based on these metrics. Preliminary results from the measurements and analysis will be presented. [Work supported by SERDP.]

\section{$1: 30$}

2pNS3. Highway noise levels in a suburban environment under inversion conditions. James Chambers (Dept. of Mech. Eng., Univ. of Mississippi, University, MS 38677), Hugh Saurenman (ATS Consultants Inc., Los Angeles, CA 90017), Robert Bronsdon, Louis Sutherland (Simi Valley, CA 93063), Ken Gilbert, Roger Waxler, and Carrick Talmadge (The Univ. of Mississippi, University, MS 38677)

Noise levels were measured in Scottsdale, AZ during a two week period in March, 2004 to identify the reasons for increased noise levels near a major highway during early morning hours. The noise levels were accompanied by meteorological measurements as well as traffic counts to fully describe the problem. The noise levels were measured in one-third octave bands and ranged $100 \mathrm{ft}$ to $2620 \mathrm{ft}(30-800 \mathrm{~m})$ from the highway and included data on both sides of the highway. The meteorological data indicated inversion conditions or downward refraction during the times of interest and model results from a Parabolic Equation (PE) calculation indicated good results with the data. The data and model indicated an approximately $10-15 \mathrm{~dB}$ increase in levels during inversion conditions which rapidly transitioned to neutral and lapsed conditions shortly after sunrise. The results of the modeling effort as well as the data will be presented. [Work supported by Arizona Dept. of Transportation.]

\section{1:45}

2pNS4. Determination of noise descriptors and criteria for pyrotechnic noise sources. Weixiong Wu (AKRF, 117 East 29th St., New York, NY 10016)

A noise study was conducted to determine appropriate noise descriptors and criteria for assessing pyrotechnic noise sources. The study was carried out to support an environmental impact statement (EIS) that defined sensitive land uses adjacent to reservoirs in New York City area, where potential noise impacts from avian dispersion measures would occur. The pyrotechnic techniques defined as impulsive noise sources are 
among the avian dispersion measures that would be used at the reservoirs. Determining appropriate noise descriptors and criteria was critical to the EIS because of the distinctive sound characteristics of pyrotechnic impulse noise sources, the lack of published literature on assessing them, and the absence of corresponding noise regulations. Noise descriptors and criteria used for EIS in the United States were investigated, and noise measurements for pyrotechnic noise sources and some impulsive noise sources were also performed. The study results demonstrate that $C$-weighted DNL is an appropriate descriptor for assessing noise impacts from the pyrotechnics based on the U.S. Army Environmental Noise Management Program criteria, and peak hour A-weighted Leq(1) is a suitable noise descriptor for determining noise impacts for avian dispersion measures, including the pyrotechnics, based on the New York City Environmental Quality Review criteria.

\section{2:00}

2pNS5. A study of automobile exhaust noise preferences. Jay B. Haire, Melinda J. Carney, and Dominique J. Cheenne (Dept. of Audio Arts \& Acoust., Columbia College Chicago, Chicago, IL 60605)

A study was conducted to investigate the relationship between preferences in automobile exhaust noise and the demographic factors of a listening jury. Noise samples of four different vehicles were recorded at idle as well as at $3000 \mathrm{RPM}$, and 1/3 octave sound spectra were acquired simultaneously. The recordings were presented to the jury using headphones and a preference survey was administered. Zwicker loudness was computed for all samples. Demographic factors such as gender, age, current and future vehicle ownership, were correlated to listening preferences, and unforeseen results were found, especially in regards to sport utility vehicles (SUV).

\section{2:15}

2pNS6. Sound-quality analysis of sewing machines. James Chatterley, Andrew Boone, Jonathan Blotter (Dept. of Mech. Eng., Brigham Young Univ., 435 CTB, Provo, UT 84602), and Scott Sommerfeldt (Brigham Young Univ., Provo, UT 84602)

Sound quality analysis procedure and results for six sewing machines ranging from entry level to professional grade will be presented. The procedure consisted of jury-based listening tests and quantification of sound quality using standard metrics. The procedures and analysis of the jury testing will be presented and discussed. The correlation between the quantitative metrics and the qualitative jury results will be presented. Sound localization scans, using near field acoustic holography techniques with accompanying results, performed in order to determine machine sound hot spots and possible sources for undesired sounds, will also be presented. Proposed modifications to machine structure in order to alter machine sound signature into a more sensory pleasant sound will also be presented.

\section{2:30}

2pNS7. Criteria for multiple noises in residential buildings using combined rating system. Jin Yong Jeon, Jong Kwan Ryu, and Young Jeong (School of Architectural Eng., Hanyang Univ., Seoul 133-791, Korea)

Multiple residential noises such as floor impact, air-borne, bathroom, drainage, and traffic noises were classified using a combined rating system developed from a social noise survey and auditory experiments. The effect of individual noise perception on the evaluation of the overall noise environment was investigated through a questionnaire survey on annoyance, disturbance, and noise sensitivity. In addition, auditory experiments were undertaken to determine the allowable sound pressure level for each residential noise source and the percent satisfaction for individual noise levels. From the results of the survey and the auditory experiments, a combined rating system was developed and annoyance criteria for multiple residential noises were suggested.
2pNS8. Noise impact on community: A case study for power generation facility. Yong Ma, Jonathan Chui, and Salem Hertil (ATCO Noise Management, 1243 McKnight Blvd. NE, Calgary, AB, Canada)

Power generation plant will make noise impact on the surrounding communities and cause noise complaints from the residences. Noise mitigation treatment for plant is required to achieve the specified noise regulations. In this paper, a case study of the noise control design for a power generation facility is presented. Major noise sources included five engines and generators, five gas conditioning skids, five radiator coolers, and other accessory equipment. The acoustic modeling software Cadna/A was used to predict the noise contributions from sources and assess the noise impact on the nearby communities. During the acoustic modeling, alternative noise mitigation measures underwent two specific investigations before they were chosen as a noise solution recommendation. The first was to determine the technical feasibility of attenuating the source equipment. The second was to perform a cost benefit analysis, necessary to find the most cost-effective solution. For example, several acoustic wall and roof assemblies were entered into the acoustic model and the acoustic performance of the ventilation system was varied until we were able to achieve the most economical acoustic solution.

\section{3:00}

2pNS9. Multi-channel active noise control on an axial fan using variable loads. Connor Duke and Scott Sommerfeldt (Brigham Young Univ., N283 ESC Provo, UT 84602)

A multi-channel active noise control system using a mock computer enclosure has been shown to produce significant reduction in tonal noise produced by an axial fan. The mock computer enclosure creates an impedance which influences the fan operation. For this system to be used commercially, it should be versatile enough to be used in a variety of enclosures and environments. Various enclosures and environments can be simulated using a fan plenum to create different back pressures on the system. The effects of different loads seen by the system on the performance of the system will be discussed. The performance of the system can be characterized in either a reverberant or anechoic environment, and the results so obtained will be presented.

\section{3:15}

2pNS10. Low-frequency noise and air vibration generated by a simple cycle gas turbine installation. Chris Giesbrecht and Salem Hertil (ATCO Noise Management, 1243 McKnight Blvd NE, Calgary, AB, Canada T2E 5T1, Chris.Giesbrecht@atconoise.com)

Standard noise regulations, measurement techniques, and acoustic treatments can fail to address energy emitted by certain problem gas turbine installations in the form of low-frequency noise and air vibrations. Survey and treatment of the entire acoustic environment is necessary to diagnose and solve these annoyance problems. The presence of groundborne vibrations, infrasound, and noise in the lower octave bands complicates environmental noise assessment and limits mitigation options. Variability in the perception, effects, and annoyance level of low-frequency noise and air vibrations prevents this relatively common problem from being well understood by industry. Particular attention must be paid to propagation paths when the dynamic range of air-borne and ground-borne vibrations overlaps. This paper is the case study of a 2002 analysis, by ATCO Noise Management, of an installation consisting of 3-120 MW combustion generator turbines causing such low-frequency noise and air vibrations. 
2pNS11. Global active control of broadband noise from small axial cooling fans. Matthew J. Green and Scott D. Sommerfeldt (Dept. of Phys. and Astron., Brigham Young Univ., N-281 ESC, Provo, UT 84602, green.mattgreen@gmail.com)

The filtered-x LMS algorithm has previously been used for feedforward control of the tonal noise of a small axial cooling fan. This system consists of four actuator-sensor pairs surrounding the small fan. The ideal placement of these elements has been previously determined by Gee and Sommerfeldt [Gee and Sommerfeldt, J. Acoust. Soc. Am. 115, 228-236 (2004)]. With success in reducing tonal noise to about the same level as broadband noise it now becomes desirable to control the broadband noise of the small axial cooling fan. This is accomplished with active feedback control. The performance of the broadband control system will be reviewed, and the results of a hybrid (feed-forward and feedback) system for overall reduction of cooling fan noise will be discussed. Ideal methods and configurations for feedback control will also be discussed.

\section{3:45}

2pNS12. A predictive noise study regarding the proposed Cincinnati Muncipal Airport expansion. Grant E. Limberg, Melinda J. Carney, and Dominique J. Cheenne (Dept. of Audio Arts \& Acoust., Columbia College Chicago.)

A noise study was performed on the landing path of runway $21 \mathrm{~L}$ of Cincinnati Municipal Airport to determine the effect of the runway expansion project set to begin in 2005. Sound pressure levels were acquired along the landing path branches to evaluate the eight-hour Leq and the test data were compared to those predicted by the Integrated Noise Model (INM) software. The test data line up to the modeled results within $3.5 \mathrm{~dB}$, an amount that can be explained by weather patterns and other environmental noise variables. The model shows that the $65 \mathrm{~dB}$ Day/Night Level (DNL) contour area could negatively affect 46 residents near the airport. Suggestions for the expansion plans include a detailed review of all relevant noise issues using an appropriate computer model.
2pNS13. An outdoor noise propagation study to predict the effect of a power plant expansion. Philip J. Brasovan, Melinda J. Carney, and Dominique J. Cheenne (Dept. of Audio Arts \& Acoust,. Columbia College Chicago, 600 S. Michigan Ave., Chicago, IL 60605)

The results of an outdoor noise propagation model using CadnaA were compared to test data obtained on-site. The subject property is the central utility plant of a hospital located in Milwaukee, scheduled to be expanded with the addition of cooling towers. The modeled area was $400 \mathrm{~m}$ squared with a resolution grid of $2 \mathrm{~m}$ squared. The model was used to validate the observed test data as well as to predict the anticipated noise levels at completion of the expansion. A total of 11 points were investigated and the predicted data were found to match the test values within $2 \mathrm{~dB}$ at many locations. The data from the model show that the anticipated noise levels at the East property line will exceed those mandated by local ordinances by $3 \mathrm{~dB}$. The model also predicts that the addition of a three meter absorbing barrier and the use of reduced noise fans for the six cell cooling system will bring the overall noise level from the system into compliance.

\section{4:15}

2pNS14. Is Lighthill's acoustic analogy still relevant? Werner Richarz (Aercoustics Eng. Ltd., 50 Ronson Dr., Ste. 165, Toronto, ON, Canada M9W 1B3)

More than fifty years after Lighthill's seminal paper, the acoustic analogy appears to be out of favor, even though the theory has been able to cope with all aspects of jet noise. This review examines the principal features of Lighthill's acoustic analogy through the lens of the self-and shear noise theory (one of several possible formulations), introduced by Proudman and extended by Ribner and others. One to infer a great deal about the sound field of a jet with a minimum of information about the details of the flow. For example, the well-known U8 scaling law is readily extended to predict the variation of the far-field power spectra of jet noise. The validity has been demonstrated by applying the prediction algorithm to measured jet noise data. Correlations of the source terms and the farfield sound have been measured and shown to exhibit closure. Subtle features such as two-point correlations of far-field sound pressures are accurately described. Recently, Ribner has proposed a means by which the elusive refraction due to flow and temperature gradients can be dealt with in a consistent manner. Predictions based thereon agree quite well with measurement, even for small angle from the jet axis. 


\title{
Session 2pPA
}

\section{Physical Acoustics, Engineering Acoustics and Noise: Infrasound: A New Frontier in Monitoring the Earth II}

\author{
Wayne N. Edwards, Cochair \\ Dept. of Earth Sciences, Univ. of Western Ontario, 1151 Richmond St., London, ON N6A 5B7, Canada \\ David E. Norris, Cochair \\ BBN Technologies, 1300 North 17th St., Arlington, VA 22209
}

\section{Contributed Papers}

$1: 30$

2pPA1. Assessment of atmospheric models for tele-infrasonic propagation. Mihan McKenna (Dept. of Geological Sci., Southern Methodist Univ., P.O. Box 750395, Dallas, TX 75275-0395, smckenna@ smu.edu) and Sylvia Hayek (Natural Resources, Canada)

Iron mines in Minnesota are ideally located to assess the accuracy of available atmospheric profiles used in infrasound modeling. These mines are located approximately $400 \mathrm{~km}$ away to the southeast (142) of the Lac-Du-Bonnet infrasound station, IS-10. Infrasound data from June 1999 to March 2004 was analyzed to assess the effects of explosion size and atmospheric conditions on observations. IS-10 recorded a suite of events from this time period resulting in well constrained ground truth. This ground truth allows for the comparison of ray trace and PE (Parabolic Equation) modeling to the observed arrivals. The tele-infrasonic distance (greater than $250 \mathrm{~km}$ ) produces ray paths that turn in the upper atmosphere, the thermosphere, at approximately $120 \mathrm{~km}$ to $140 \mathrm{~km}$. Modeling based upon MSIS/HWM (Mass Spectrometer Incoherent Scatter/ Horizontal Wind Model) and the NOGAPS (Navy Operational Global Atmospheric Prediction System) and NRL-GS2 (Naval Research Laboratory Ground to Space) augmented profiles are used to interpret the observed arrivals.

\section{$1: 45$}

2pPA2. Infrasound radiation of cyclones. Konstantin A. Naugolnykh (NOAA/ETL/Zeltech, 325 Broadway, Boulder, CO 80305)

Tropical cyclones produce strong perturbations of atmosphere and the ocean surface accompanied by acoustical radiation. Infrasonic signals in the $0.1-0.5$ frequency band can be observed at distances of thousands of miles from the cyclone. The effect of infrasound radiation is connected apparently to the interaction of the counter-propagating sea-surface waves that produces a sound radiation of the doubled frequency of the surface wave oscillation. This radiation has narrow-angle vertical directivity pattern. The essential refraction of radiated infrasound in the atmosphere perturbed by the cyclone leads to trapping of the infrasound by the horizontal atmospheric wave-guide providing its long distance propagation.

\section{2:00}

2pPA3. Characteristics of infrasound signals from earthquakes. Paul Mutschlecner and Rodney Whitaker (Los Alamos Natl. Lab., EES-2 MS J577, LANL, Los Alamos, NM 87545)

Analysis of infrasound signals is presented for a set of 31 earthquakes located mostly in the western United States and recorded at infrasound arrays operated by the Los Alamos National Laboratory. By normalizing measured amplitudes for the effects of propagation and distance, a pressure-amplitude versus earthquake magnitude relation is derived. Further analysis showed that the observed variance in this relation is likely due to the variation in source ground motion and variation in upper atmospheric winds. Signal durations can be tens of minutes and azimuth devia- tions have a mean of three degrees (measured from array data compared to great circle azimuths). Other characteristics of the infrasound data will be discussed. Our analysis of the observed data combined with surface ground motion accelerations, led to estimates of the minimum surface accelerations needed to generated infrasound signals measured at distant arrays.

\section{$2: 15$}

2pPA4. An analysis of seismic and acoustic signals from the June 3, 2004 Washington state bolide. Stephen Arrowsmith, Michael Hedlin (IGPP, Scripps, Univ. of California, San Diego, La Jolla, CA 92093-0225), Lars Ceranna (BGR, Hannover 30625, Germany), and Wayne Edwards (Univ. of Western Ontario, Canada, N6A 3K7)

On June 3rd, 2004 a spectacular bolide was reported over British Columbia, Washington, Oregon and Idaho. In addition to eyewitness accounts and video recordings, the event was recorded on a number of seismometers in the Pacific Northwest Seismograph network and at infrasound arrays in Washington State and California. Using the NRL-G2S atmospheric model for the time of the event, source locations have been determined with seismic and acoustic signals separately. Estimates of the yield and acoustic efficiency of the explosion have also been determined. By simulating the propagation of infrasound through the atmosphere, the arrival of discrete acoustic phases at the infrasound arrays has been modeled.

$2: 30$

2pPA5. Acoustic observations of large earthquakes and associated phenomena. Catherine de Groot-Hedlin (Scripps Inst. of Oceanogr., UCSD, 9500 Gilman Dr., La Jolla, CA 92093-0225, chedlin@ucsd.edu) and David McCormack (Geological Survey of Canada, Ottawa, ON, Canada K1A 0Y3)

Recent global events have renewed interest in acoustic observations of large earthquakes and associated phenomena such as landslides and tsunamis. In addition, the proliferation of global networks of atmospheric acoustic (infrasound) and hydroacoustic observing systems mean that signals from such events are captured in close to real-time more frequently and with better resolution than ever before. Such data provide a useful complement to more traditional seismological observations of such events. In this paper, recent acoustic and hydroacoustic observations from large earthquake events are presented. As well as the scientific insights provided by use of such monitoring systems, operational applications useful for hazard mitigation or hazard alerting are considered. Current plans to develop observing systems to perform systematic studies of areas susceptible to earthquake and tsunami hazard offshore western Canada, and the use of such proposed systems to make systematic studies of hydroacoustic signals from seismic events, underwater slumping and landslides are described. 
2pPA6. High trace-velocity events associated with auroral activity in Alaska. Charles R. Wilson and John V. Olson (Geophysical Inst., Univ. of Alaska, Fairbanks, AK 99775)

Data from the CTBT/IMS station 153US at Fairbanks, AK covering 2003 and 2004 have been surveyed for high-correlation, high tracevelocity events. Although such events have been detected at all times of the day the distribution shows a strong maximum in the morning hours (near 15UT) and a weaker maximum in the evening hours (near 5UT). Twenty intervals containing high trace-velocity events from 2003 and 37 intervals from 2004 have been studied in detail. The intervals containing high trace-velocity events can last from a few hours up to 10 hours. Allsky video records show that the high trace-velocity events are associated with stable, pulsating auroral patches that occur during the recovery phase of substorms. This talk will review the characteristics of the high tracevelocity events and the associated auroral video where available.

\section{3:00-3:30 Break}

\section{$3: 30$}

2pPA7. Source localization of non-stationary acoustic data using time-frequency analysis. Jack Stoughton (NASA-Langley Res. Ctr., MS 488, Hampton, VA) and William Edmonson (Hampton Univ., Hampton, VA 23668)

An improvement in temporal locality of the generalized crosscorrelation (GCC) for angle of arrival (AOA) estimation can be achieved by employing 2-D cross-correlation of infrasonic sensor data transformed to its time-frequency (TF) representation. Intermediate to the AOA evaluation is the time delay between pairs of sensors. The signal class of interest includes far field sources which are partially coherent across the array, nonstationary, and wideband. In addition, signals can occur as multiple short bursts, for which TF representations may be more appropriate for time delay estimation. The GCC tends to smooth out such temporal energy bursts. Simulation and experimental results will demonstrate the improvement in using a TF-based GCC, using the Cohen class, over the classic GCC method. Comparative demonstration of the methods will be performed on data captured on an infrasonic sensor array located at NASA Langley Research Center (LaRC). The infrasonic data sources include Delta IV and Space Shuttle launches from Kennedy Space Center which belong to the stated signal class. Of interest is to apply this method to the AOA estimation of atmospheric turbulence. [Work supported by NASA LaRC Creativity and Innovation project: Infrasonic Detection of Clear Air Turbulence and Severe Storms.]

\section{$3: 45$}

2pPA8. Multiparameter studies of surf infrasound. Milton Garces, David Fee, Pierre Caron, Claus Hetzer (Infrasound Lab., Univ. of Hawaii, Manoa, 73-4460 Queen Kaahumanu Hwy., \#119, Kailua-Kona, HI 96740-2638), Jerome Aucan, Mark Merrifield (Univ. of Hawaii, Manoa, Honolulu, HI 96822), Robert Gibson, and Joydeep Bhattacharyya (BBN Technologies, Arlington, VA 22209)

Infrasound stations on islands or near coastlines routinely detect signals associated with breaking ocean waves. Although the source mechanisms of these infrasonic surf signals are not well understood, they might provide useful insight into coastal processes and permit an assessment of wave energy distribution in the littoral zone. Near-shore infrasonic arrays, ocean bottom sensors, an infrared imager, and a video camera were deployed on rocky and sandy coastlines in Hawaii during the 2004-2005 Winter swell season, with the aim of establishing a relationship between the directional ocean swell height and the infrasonic source distribution, intensity, and spectral content. The cameras targeted acoustically active regions for selected time periods to associate the timing of the infrasonic signal arrivals with the breaking and dissipation of ocean wave sets. A wide range of sea states and weather conditions were captured during two separate deployments. The results of our multiparameter analyses and preliminary comparisons of our observations with mesoscale ocean wave model predictions will be presented. [Work supported by the Defense Advanced Research Projects Agency. Support of the State of Hawaii is acknowledged.]

\section{4:00}

2pPA9. Multiple array infrasound observations in the Netherlands. Läslo Evers and Hein Haak (Royal Netherlands Meteorological Inst. (KNMI), P.O. Box 201, 3730 AE De Bilt, The Netherlands, evers@knmi.nl)

Infrasound in the Netherlands is observed with multiple arrays. The current architecture consists of 4 arrays. These arrays vary in aperture from 30 to $1500 \mathrm{~m}$ while the number of microbarometers ranges from 6 to 16 per array. Continuous observation of infrasound implies a huge number of automatic detections per year. In other words, thousands of coherent waves cross the arrays each year. Although a lot of sources can be identified, there are still unknown sources having a coherent infrasonic signature. Among the identified sources are: supersonic airplanes, bolides, severe weather, oceanic waves, volcano explosions, and military activity. Current efforts in source identification concentrate on the integration of infrasonic and seismic data. Furthermore, atmospheric characteristics are included such as the state of the boundary layer and propagation conditions in the higher atmospheric, i.e., stratospheric winds and temperatures. In this presentation the influence of the atmosphere on the detection capability will be shown. Furthermore, the detailed analysis of specific sources shows the enormous potential of infrasound as atmospheric probe and monitoring technique.

\section{$4: 15$}

2pPA10. Status report on the establishment of the CTBTO IMS infrasound network. Thomas L. Hoffmann (CTBTO IMS, Vienna Intl. Ctr., P.O. Box 1200, A-1400 Vienna, Austria)

Steady progress has been made in the establishment of the CTBTO IMS infrasound monitoring network. To date $86 \%$ of the site surveys for 60 infrasound stations in the network have been completed, $50 \%$ of the stations are transmitting continuous data to Vienna, and $40 \%$ of the stations have been certified. While the global distribution pattern of infrasound stations transmitting data to Vienna is still disperse, regional networks begin to form in North and South America as well as in the Australian and South African regions. This presentation will focus on an overview of recent progress made in the establishment of the global infrasound network, and also present some of the challenges and difficulties encountered in this program. 


\title{
Session 2pPP
}

\section{Psychological and Physiological Acoustics: Psychoacoustics: In Memory of Søren Buus}

\author{
Bertram Scharf, Cochair \\ Northeastern Univ., Psychology, Boston, MA 02115-5096 \\ Rhona P. Hellman, Cochair \\ Northeastern Univ., Speech Language Pathology and Audiology, 360 Huntington Ave., Boston, MA 02115
}

Chair's Introduction-1:00

Invited Papers

1:05

2pPP1. Søren Buus. Thirty years of psychoacoustic inspiration. Torben Poulsen (Oersted-DTU, Acoust. Technol., Tech. Univ. of Denmark, DK 2800 Lyngby, Denmark, tp@oersted.dtu.dk)

Søren Buus did his MSc at the Acoustics Laboratory, Technical University of Denmark (DTU), in 1975 on the topic headphone calibration. He showed the importance of reliable reference values for psychoacoustic research and Søren was a great inspiration for my work [Scand. Audiol. 20, 205-207 (1991); 27, 105-112 (1998)]. Already from the seventies, temporal integration of loudness has been a major topic in the collaboration with Søren [Buus et al., J. Acoust. Soc. Am. 105, 3464-3480 (1999)] and the measurements of temporal integration over a wide range of presentation levels led to the important finding about the shape of the loudness function [Buus et al., J. Acoust. Soc. Am. 100, 669-680 (1997)]. Søren talked about the importance of the psychophysical procedure and the influence from the procedure on the results [Buus, Proceedings 19th Danavox Symposium (2001), pp. 183-226]. The goal was to obtain reliable, unbiased, and precise results. An overview of some of the above investigations will be presented together with recent results from a MSc project on headphone calibration of short duration sounds for ABR measurements.

$1: 30$

2pPP2. Søren Buus' contribution to speech intelligibility prediction. Hannes Müsch (Sound ID, Palo Alto, CA 94303, hmuesch@soundid.com) and Mary Florentine (Northeastern Univ., Boston, MA 02115)

In addition to his work in psychoacoustics, Søren Buus also contributed to the field of speech intelligibility prediction by developing a model that predicts the results of speech recognition tests [H. Müsch and S. Buus, J. Acoust. Soc. Am. 109, 2896-2909 (2001)]. The model was successful in test conditions that are outside the scope of the Articulation Index. It builds on Green and Birdsall's concept of describing a speech recognition task as selecting one of several response alternatives [in D. Green and J. Swets, Signal Detection Theory (1966), pp. 609-619], and on Durlach et al.'s model for discriminating broadband sounds [J. Acoust. Soc. Am. 80, 63-72 (1986)]. Experimental evidence suggests that listeners can extract redundant, independent, or synergistic information from spectrally distinct speech bands. One of the main accomplishments of the model is to reflect this ability. The model also provides for a measure of linguistic entropy to enter the intelligibility prediction. Recent model development has focused on investigating whether this measure, the cognitive noise, can account for the effects of semantic and syntactic context. This presentation will review the model and present new model predictions. [Work supported by NIH grant R01DC00187.]

\section{$1: 55$}

2pPP3. Borrowing a trick from Sbren Buus: Application of a simple quantitative model to data obtained from an impaired population. Robert P. Carlyon (MRC Cognition and Brain Sci. Unit, 15 Chaucer Rd., Cambridge, CB2 2EF, England)

One of the many things that Sbren taught me was to apply a simple quantitative model to psychophysical data, even when studying impaired populations, renowned for inter-listener variability. Here I present a recent application of this approach, applied to cochlear implant patients' detection thresholds for electrical stimulation. Most implants stimulate the nerve with trains of biphasic pulses, in which a rectangular pulse of one polarity is followed, with a short inter-phase gap (IPG), by an equal pulse of opposite polarity. Together with van Wieringen and colleagues in Leuven, Belgium, we have shown that psychophysical thresholds in humans drop as IPG increases to at least 2900 microseconds implying a much longer time constant than predicted from physiological studies on animals. By applying a simple model, we showed that this long time constant is quantitatively consistent with the threshold drop observed for electrical sinusoids as frequency is lowered to about $100 \mathrm{~Hz}$. The model involves passing the electrical waveform through a low-pass filter and calculating the RMS output. Despite its simplicity, the model successfully predicts the results of several novel manipulations, and helps constrain explanations of the physiological basis of the observed time constants. 
2pPP4. Sequential grouping constraints on across-channel auditory processing. Andrew J. Oxenham (Res. Lab. of Electron., MIT, Cambridge, MA 02139, oxenham@mit.edu) and Torsten Dau (Tech. Univ. of Denmark, DK-2800 Lyngby, Denmark)

Søren Buus was one of the pioneers in the study of across-channel auditory processing. His influential 1985 paper showed that introducing slow fluctuations to a low-frequency masker could reduce the detection thresholds of a high-frequency signal by as much as $25 \mathrm{~dB}$ [S. Buus, J. Acoust. Soc. Am. 78, 1958-1965 (1985)]. Sbren explained this surprising result in terms of the spread of masker excitation and across-channel processing of envelope fluctuations. A later study [S. Buus and C. Pan, J. Acoust. Soc. Am. 96, 1445-1457 (1994)] pioneered the use of the same stimuli in tasks where across-channel processing could either help or hinder performance. In the present set of studies we also use paradigms in which across-channel processing can lead to either improvement or deterioration in performance. We show that sequential grouping constraints can affect both types of paradigm. In particular, the perceptual segregation of off-frequency from on-frequency components, using sound sequences preceding or following the target, leads to results similar to those found in the absence of the off-frequency components. This suggests a high-level locus for some across-channel effects, and may help provide a functional distinction between within- and across-channel mechanisms. [Work supported by NIH R01DC03909 and Danish Research Council.]

\section{2:45-3:00 Break}

\section{3:00}

2pPP5. Tone-burst otoacoustic emissions and loudness. Michael Epstein (Inst. of Hearing, Speech and Lang., Comm. Res. Lab, and Comm. and DSP Ctr., ECE Dept. (440 DA), Northeastern Univ., Boston, MA 02115, mepstein@ece.neu.edu) and Mary Florentine (Northeastern Univ., Boston, MA 02115)

Several models of cochlear mechanics lead to the conclusion that the amplitude of tone-burst otoacoustic emissions (TBOAEs) is proportional to basilar-membrane (BM) motion. Buus and Florentine [Fechner Day 2001 (Pabst, Berlin), 236 (2001)] showed that the square of loudness, derived from measurements of spectral and temporal integration, closely matched basilar-membrane velocity at the best frequency. Buus et al. [Physiological and Psychophysical Bases of Auditory Function, 373 (2001)] examined the relationship between distortion-product otoacoustic emissions (DPOAEs) and BM motion. Follow-up work by Epstein et al. [J. Acoust. Soc. Am. 117, 263 (2005)] showed in a direct comparison that TBOAEs and psychoacoustical measures, of loudness and of pulsation threshold, on six subjects with normal hearing led to very similar estimations of basilar-membrane motion. These outcomes suggest that otoacoustic emissions could serve as an excellent tool-one that is objective, non-invasive, and rapid-for estimating in subjects with normal hearing both relative basilar-membrane motion and relative loudness. [Work supported by NIH/NIDCD Grant R01DC02241.]

2pPP6. Induced loudness reduction: A review. Bertram Scharf (Psychol. Dept., Northeastern Univ., Boston, MA 02115), Eva Wagner, and Bärbel Nieder (Northeastern Univ., Boston, MA 02115, scharf@neu.edu)

Under appropriate stimulus conditions, a tone may decline in loudness the equivalent of $10 \mathrm{~dB}$ and more when preceded by a stronger tone. This induced loudness reduction or ILR was uncovered, indirectly, in a large number of studies by L. E. Marks and his associates [e.g. Marks, J. Exp Psychol HPP 20, 382-396 (1994)]. Those studies seemed to suggest that ILR required that tones be presented at two widely separated frequencies over a relatively large range of SPLs. Although part of the measured loudness changes seemed to stem from response biases, recent studies show unequivocally that the reduction in loudness is mostly sensory and that tones need be presented at only a single frequency and at two levels some 10 to $20 \mathrm{~dB}$ apart. The present paper puts together what is known about the dependence of ILR on signal frequency, level, duration, temporal relations, and hearing loss. The role of ILR in various other psychoacoustical phenomena such as induced loudness adaptation and loudness enhancement is reviewed. Much of this knowledge was obtained in direct and indirect collaboration with Søren Buus. [Work supported by NIH/NIDCD Grant No. R01 DC 02241.]

\section{Contributed Paper}

\section{3:50}

2pPP7. Extension of Sbren Buus's modeling to loudness growth at high frequencies. Rhona P. Hellman (Dept. of Speech-Lang. Path. and Audiol. and Inst. for Hearing., Speech, \& Lang. (106A FR), Northeastern Univ., Boston, MA 02115, hellman@neu.edu)

Loudness matching between tones at different frequencies leads to results that imply that loudness functions, which relate loudness to sound pressure level (SPL) are parallel at frequencies from 1 to $10 \mathrm{kHz}$ but not at higher frequencies [Hellman et al., J. Acoust. Soc. Am. 109, 2349 (2001)]. Within the 12.5 to $16 \mathrm{kHz}$ frequency range, the loudness-matching func- tions are curvilinear in shape being steeper below 60 phons than at higher loudness levels. The higher the frequency, the greater is the decrease in slope above 60 phons. The slope reduction above 60 phons for frequencies from 12.5 to $16 \mathrm{kHz}$ is ascribed to the tone's restricted excitation pattern. A computer model by Søren Buus described in part in Florentine et al. [Modeling Sensorineural Hearing Loss (Erlbaum, 1997), pp. 187-198] provides a good account of the decrease in the mid-to-high level slope of loudness functions derived at high frequencies from the matching data. The agreement between the model predictions and the empirical results further supports the notion that the rate of loudness growth at moderateto-high SPLs depends on the upward spread of excitation. 


\title{
Session 2pSA
}

\section{Structural Acoustics and Vibration: General Vibration; Excitation, Radiation, and Dampening}

\author{
Kenneth D. Frampton, Chair \\ Dept. of Mechanical Engineering, Vanderbilt Univ., Nashville, TN 37235-1592
}

\section{Contributed Papers}

2:30

2pSA1. Non-contact mode excitation of small structures in air using ultrasound radiation force. Thomas M. Huber, John C. Purdham (Dept. of Phys., Gustavus Adolphus College, 800 College Ave., St. Peter, MN 56082, huber@gustavus.edu), Mostafa Fatemi, Randall R. Kinnick, and James F. Greenleaf (Mayo Clinic College of Medicine, Rochester, MN 55905)

With the advent of MEMS, modal analysis of small structures is increasingly important. However, conventional excitation techniques normally require contact, which may not be feasible for small objects. We present a non-contact method that uses interference of ultrasound frequencies in air to produce low-frequency excitation of structures. Objects studied included hard-drive HGA suspensions and MEMS devices. The vibration induced by the ultrasound radiation force was varied in a wide range from $0 \mathrm{~Hz}$ to $50 \mathrm{kHz}$. Object motion was detected using a laser vibrometer; measured frequencies agreed with expected values. Also demonstrated was the unique capability to selectively enhance or suppress modes independently. For example, the ratio of the vibrational amplitudes of the 175 $\mathrm{Hz}$ first-bending and $1.33 \mathrm{kHz}$ torsional modes of a small cantilever could be changed from in excess of 10:1 to less than 1:10 by shifting the ultrasound modulation phase 90 degrees. Similar changes were obtained for a $3 \mathrm{~mm}$ square MEMS mirror in the ratios of vibration amplitude around its two separate axes. Torsional modes of a hard-drive suspension could be selectively enhanced by over a factor of two by moving the ultrasound focus point from near the center to near the edge of the suspension.

\section{2:45}

2pSA2. Direction selective structural-acoustic coupled radiator. HeeSeon Seo and Yang-Hann Kim (NOVIC, KAIST, 373-1 Sci. Town Daejon-si, Korea)

This paper presents a method of designing a structural-acoustic coupled radiator that can emit sound in the desired direction. The structural-acoustic coupled system is consisted of acoustic spaces and wall. The wall composes two plates and an opening, and the wall separates one space that is highly reverberant and the other that is unbounded without any reflection. An equation is developed that predicts energy distribution and energy flow in the two spaces separated by the wall, and its computational examples are presented including near field acoustic characteristics. To design the directional coupled radiator, Pareto optimization method is adapted. An objective is selected to maximize radiation power on a main axis and minimize a side lobe level and a subjective is selected direction of the main axis and dimensions of the walls geometry. Pressure and intensity distribution of the designed radiator is also presented.

\section{3:00}

2pSA3. Development of a directivity controlled piezoelectric transducer for sound reproduction. Magella Bédard and Alain Berry (G.A.U.S., Dept. of Mech. Eng., Université de Sherbrooke, 2500, boul. de l'Université, Sherbrooke, Québec, Canada, J1K 2R1, magella.bedard@usherbrooke.ca)

One of the inherent limitations of loudspeaker systems in audio reproduction is their inability to reproduce the possibly complex acoustic directivity patterns of real sound sources. For music reproduction for example, it may be desirable to separate diffuse field and direct sound components and project them with different directivity patterns. Because of their properties, poly (vinylidene fluoride) (PVDF) films offer lot of advantages for the development of electroacoustic transducers. A system of piezoelectric transducers made with PVDF that show a controllable directivity was developed. A cylindrical omnidirectional piezoelectric transducer is used to produce an ambient field, and a piezoelectric transducers system, consisting of a series of curved sources placed around a cylinder frame, is used to produce a sound field with a given directivity. To develop the system, a numerical model was generated with ANSYS Multiphysics TM8.1 and used to calculate the mechanical response of the piezoelectric transducer. The acoustic radiation of the driver was then computed using the Kirchoff-Helmoltz theorem. Numerical and experimental results of the mechanical and acoustical response of the system will be shown.

\section{3:15}

2pSA4. Progress towards an electro-acoustic resonance technique for determining quantitative material and geometrical properties in high contrast multi-layer elastic structures.. Karl Fisher (7000 E. Ave, Livermore, CA 94551)

High contrast multilayered elastic structures continue to be problematic for ultrasonic inspection. Large acoustic material impedance mismatches, refraction, reverberation, multiple echoes, and high elastic attenuations are just some of the issues one is faced with standard high frequency $(1-20 \mathrm{MHz})$ pulse echo detection and imaging methods. In this presentation, we will present progress towards developing a low frequency resonance technique that operates in the 20 to $70 \mathrm{kHz}$ regime. The technique is based on a direct correlation between the electrical impedance of a standard electro-acoustic transducer and the mechanical loading it experiences when placed in contact with a layered elastic structure. Preliminary experimental and theoretical results are in good agreement.

$$
\text { 3:30 }
$$

2pSA5. A simple model for coupled acoustic-structure resonance in Stratospheric Observatory for Infrared Astronomy. Jerry H. Ginsberg (G. W. Woodruff School of Mech. Eng., Georgia Inst. of Technol., Atlanta, GA 30332-0405)

The Stratospheric Observatory For Infrared Astronomy (SOFIA) is a joint project of NASA and the Deutsches Zentrum fur Luft- und Raumfahrt that has mounted a $2.5 \mathrm{~m}, 20000 \mathrm{~kg}$ infrared telescope on a bulkhead of a specially modified Boeing 747-SP. A large sliding door will expose the observation bay to the exterior flow field at Mach 0.85 and $13 \mathrm{~km}$ altitude. In the open configuration the interaction of turbulence vortices generated at the leading and trailing edges of the opening has the possibility of inducing a strong acoustic signal. A concern has been raised that the peak frequencies of such a signal might coincide with the cavity resonances. The present work examines the transfer function for a known source in order to identify the cavity resonances. Simplistic reasoning argues that the worst case would occur if the cavity resonant frequencies are close to structural resonances. However, the structure's impedance is very low at its resonances, which means that the cavity resonant frequencies are shifted from their nominal values. The present work uses a simple one-dimensional waveguide model, in which one end is terminated by a damped single-degree-of-freedom oscillator, to explain the coupled-fluid 
structure resonance. The characteristic equation and formulas for the pressure and displacement transfer functions are derived. Analysis of these results leads to some surprising insights regarding the role of a structure's stiffness and mass. [Work supported by the NASA.]

$$
\text { 3:45-4:00 Break }
$$

\section{4:00}

2pSA6. Non-intrusive ground-contacting vibrometer for acoustic/ seismic landmine detection. James S. Martin, Gregg D. Larson (Georgia Inst. of Tech., Atlanta, GA 30332-0405), and Waymond R. Scott, Jr. (Georgia Inst. of Tech., Atlanta, GA 30332-0250)

The detection of buried landmines using seismic waves and full wavefield measurements has been demonstrated. The technique requires a sensor that is non-intrusive with sufficient fidelity, reproducibility, and noise immunity for imaging processes. This has been accomplished in the past with non-contact techniques. For reasons of cost and scalability in large arrays, ground-contacting sensors are currently of interest as an alternative to these. A ground-contacting sensor configuration was studied in which an accelerometer was coupled to the ground through a viscoelastic layer with a bias force provided by a soft coil spring. This sensor was found to meet the noise, fidelity, and reproducibility requirements of a seismic landmine detection system operating in a laboratory experimental model. The fidelity of this sensor was found to vary with the bias force because of the nonlinear stiffness of the soil surrogate in the model. This dependence was sufficiently weak that no feedback of the bias force was necessary to reproducibly couple the sensor over a flat surface. The sensor offers the potential benefit of information that was not available from non-contact measurements regarding the in-plane motion of the soil surface. This data may provide additional cues for the detection of buried mines. [Work supported by ONR.]

\section{4:15}

2pSA7. Vibro-acoustic response of convected fluid loaded plates. Kenneth Frampton (Dept. of Mech. Eng., Vanderbilt Univ., Nashville, TN 37235)

This work demonstrates the effects that fluid convection has on the vibro-acoustic response of rectangular plates. The effects of fluid flow on the vibration and stability of plates is reasonably well understood. These effects include dramatic structural modal coupling along with static and dynamic instabilities. However, the resulting effect on sound radiation is not so well understood. The presentation will include a description of the fundamental physics associated with a simply supported, vibrating, rectangular plate in an infinite baffle and radiating into a semi-infinite, convected fluid. Then, simulation results will demonstrate the effects of flowinduced modal coupling and acoustic radiation. It will be demonstrated that convection can significantly increase the radiated sound power and dramatically affect the vibro-acoustic response.

\section{$4: 30$}

2pSA8. Behavior of the intermediate and exterior layers of a close fitting enclosure surrounding a cylindrical fluid-loaded acoustic source. Joseph Cuschieri (Lockheed Martin MS2, Perry Technologies, 100 East 17th street, Riviera Beach, FL 33404)

Previously, results for the Insertion Loss (IL) of a close fitting enclosure surrounding a submerged (in water) cylindrical acoustic source [Cuschieri, J. Acoust. Soc. Am. 115, 2537 (2004); 116, 2521 (2004)] were presented for different intermediate layer materials, different characteristics of the exterior cladding layer material and different source sizes. The IL results were presented as a function of frequency and layer/cladding characteristics. In this presentation, details on the behavior of the intermediate and outside layers enclosing the cylindrical source are presented which demonstrate the coupling between the cylindrical source the exter- nal acoustic (water) medium. These results help to explain the IL results previously obtained and presented. Furthermore, the scattering characteristics of the cylindrical source with and without the close fitting enclosure are considered. [Work supported by ONR.]

\section{$4: 45$}

2pSA9. A focused multi-layered spherical shell with guided wave enhancements. John D. Smith (Dstl. Porton Down, Salisbury, SP4 0JQ, UK) and Duncan P. Williams (Dstl. Winfrith, Dorchester DT2 8WX, UK)

There is a continued need for underwater reflective targets to be used as relocation and navigational aids. Traditionally, fluid-filled thin spherical shells have been used as passive reflectors, similar to cat's eyes, and would usually be filled with liquid chloroflourocarbons (CFC's). Other options are needed now that the production of CFC's has been restricted. This paper looks at the relationship between a system of multi-layered concentric elastic shells and their target strength in water. The problem is formulated using the global matrix method and is applicable to any system with an arbitrary number of layers. Results show that, by using certain combinations of low loss elastic layers together, the target strength is higher than it would be for any one of the materials used in isolation. This result is explained by geometric focussing on the back surface of the sphere and guided elastic waves that circumnavigate the outer layer or shell and enhance the focussing. The results are compared with calculations and existing experiments for a stainless steel shell filled with CFC.

\section{5:00}

2pSA10. Sound radiation of a plate excited by an impact. Physical and perceptual comparisons between numerical and experimental results. Dominique Habault, Florence Demirdjian, Sabine Meunier, and Georges Canevet (CNRS-LMA, 13402 Marseille Cedex 20, France)

The study is concerned with the response of a thin elastic baffled plate, immersed in a fluid, and excited by an impact force. An experiment was run, in which a plate was struck by an impact hammer which provides excitations of very short duration. Various shapes of excitation functions were obtained by using different kinds of hammer heads (rubber, plastic, metal). The resulting acceleration on the plate and sound pressure radiated in the fluid were measured, with an emphasis on the very first portion of the signals (initial $30 \mathrm{~ms}$ ). The recorded signals were then compared, in the time domain, with theoretical predictions based on expansions in resonance modes of the fluid-loaded plate. These predictions provide a quite accurate description of the experimental data. Auditory tests were also run, to compare the signals obtained from the calculations and the recorded sounds. Subjects were asked to evaluate the dissimilarity between test sounds, using an analog scale graduated from 0 (very similar) to 6 (very dissimilar). The tests allowed to identify the perceptual criteria used by the subjects. The aim of the study is to use these criteria to improve the numerical method of prediction of the sound radiation.

\section{$5: 15$}

2pSA11. A variational approach to modeling the vibration of timber joist floors. Colin Fox and Hyuck Chung (Mathematics Dept., Univ. of Auckland, PB 92019, Auckland, New Zealand, fox@math.auckland.ac.nz)

We present a comprehensive variational model for the vibration of timber joist floors and a simple computer algorithm for finite, particularly rectangular, floors. We allow for floor constructions that are typical in the New Zealand context, consisting of edge-supported timber joists with flooring material above, often a suspended ceiling below with absorptive material in the cavity, and a range of joint types such as gluing or nailing. The model and algorithm are structured in such a way that a component can easily be either added to, or removed from, the structure. Hence the configuration may be made progressively more complex from the simplest floor-joists type to floor-joists-ceiling with cavity air and damper-spring connectors. 


\title{
Session 2pSC
}

\section{Speech Communication: Cross-Linguistic and Dialectical Studies (Poster Session)}

\author{
Richard A. Wright, Chair \\ Dept. of Linguistics, Univ. of Washington, Seattle, WA 98195-4340
}

\section{Contributed Papers}

\begin{abstract}
All posters will be on display from 1:00 p.m. to 4:30 p.m. To allow contributors an opportunity to see other posters, contributors of odd-numbered papers will be at their posters from 1:00 p.m. to 2:15 p.m. and contributors of even-numbered papers will be at their posters from 2:15 p.m. to 4:30 p.m.
\end{abstract}

2pSC1. Free choice task effects in cross-linguistic stop perception. Alexei Kochetov (Dept. of Linguist., Simon Fraser Univ., 8888 Univ. Dr., Burnaby, BC, Canada V5A 1S6, alexei_kochetov@sfu.ca)

This paper examines the identification of stop place and secondary articulation using a free choice task. Russian syllable-initial and syllablefinal stops /p pj t tj/ in nonsense utterances were presented to Russian and Japanese listeners $(N=30)$. Correct identification rates for place and secondary articulation of the target consonants were determined based on written responses (in Cyrillic or Katakana). Both groups of listeners showed better identification of syllable-initial stops compared to syllablefinal stops. Among the consonants, /p/ was identified better, and /pj/ was identified worse than the other stops. Native listeners performed better than non-native listeners. The overall correct identification rates were lower than (yet strongly correlated with) the rates previously obtained with the same stimuli using a forced choice phoneme identification task. The lower identification rates in the current study can be explained in part by the errors involving the segmentation and syllabification of palatalized stops. Thus, the palatal articulation of the syllable-final palatalized /pj/ was often interpreted as independent of the stop (e.g., /tapj api/ rendered as /taj papi/ or /tjap api/). It is concluded that the free choice task can successfully complement the forced choice task, providing additional information about the perception of secondary palatalization. [Supported by SSHRC.]

2pSC2. The preliminary study about neutral tone: Dialect effect between North Official Mandarin speakers in China and Taiwan Mandarin speakers. Jennifer Li (Dept. of English Lit., Natl. Chiao Tung Univ., 1001 Ta Hsueh Rd., Hsinchu, Taiwan 300, Republic of China, wahaha_jennifer@yahoo.com.tw)

According to general theories, neutral tone is not regarded as an independent tone in Mandarin. Previous research shows that the most important characteristic of the neutral tone is that it does not have a certain target and pitch contour. (Lin and Yang, 1980) Namely, its pitch contour is uncertain, and it is weak in perception level. However, those studies ignore the variant between different dialects. Our study examined the features of the neutral tone between two dialects of Mandarin speakers and aimed at figuring out the dialect difference effect on the pronunciation of the neutral tone. Our subjects were chosen form two groups of Mandarin speakers. One group is from the North Mainland China, and the other is from Taiwan. The experiment was designed with a speak-it-out process. All subjects read a randomized script written in Mandarin, and the whole process was recorded spontaneously. The preliminary result shows that the dialect difference effect actually matters. It shows a tendency that the neutral tone has a certain target in Taiwan Mandarin speakers.
2pSC3. The phonetic rhythm/syntax headedness connection: Evidence from Tagalog. Sonya Bird, Laurel Fais, and Janet Werker (Univ. of Victoria and Univ. of British Columbia, Canada, sbird@uvic.ca)

Ramus, Nespor, and Mehler [Cognition (1999)] show that the rhythm of a language (broadly: stress- versus syllable- versus mora-timing) results from the proportion of vocalic material in an utterance $(\% \mathrm{~V})$ and the standard deviation of consonantal intervals (delta-C). Based on 14 languages, Shukla, Nespor, and Mehler [submitted] further argue that rhythm is correlated with syntactic headedness: low $\% \mathrm{~V}$ is correlated with headfirst languages (e.g., English); high \%V is correlated with head-final languages (e.g., Japanese). Together, these proposals have important implications for language acquisition: infants can discriminate across rhythm classes [Nazzi, Bertoncini, and Mehler, J. Exp. Psych: Human Perception and Performance (1998)]. If rhythm, as defined by $\% \mathrm{~V}$ and delta-C, can predict headedness, then infants can potentially use rhythm information to bootstrap into their languages syntactic structure. This paper reports on a study analyzing rhythm in a language not yet considered: Tagalog. Results support the Shukla et al. proposal in an interesting way: based on its \% V and delta-C, Tagalog falls between head-first and head-last languages, slighty closer to the head-first group. This placement correlates well with the fact that, although Tagalog is said to be primarily head-first syntactically, head-last phrases are permitted and common in the language.

2pSC4. A comparison of the acoustic characteristics of American English and Cantonese vowels. Eric Zee (Dept. of CTL, City Univ. of Hong Kong, 83 Tat Chee Ave., Hong Kong, ctlzee@cityu.edu.hk)

The study compares the formant frequencies of the American English vowels $[\mathrm{i}, \varepsilon, \mathrm{a}$, ○, u, I, U] (Peterson and Barney, 1952) and [i, $\varepsilon, \mathrm{a}$, ○, u, I, U] in Cantonese (Zee, 2004). Results of the comparison show that the differences in formant values for the vowels between the two languages vary according to vowel type and gender. Between male speakers of the two languages, the differences in $F$-values are minimal for [i] and [0]. For $[\varepsilon, \mathrm{u}]$, the difference between the two groups is mainly in $F 2$, with $[\varepsilon]$ having a larger $F 2$ and [u] a smaller $F 2$ for Cantonese speakers. [a] in American English has smaller $F 1$ and $F 2$ than [a] in Cantonese. [I, U] occupy the position in between the level of $[\mathrm{i}, \mathrm{u}]$ and level of $[\varepsilon, \nu]$ in the $F 1 / F 2$ plane for American English speakers, and there is a noticeable difference in $F 2$ between [I] and $[\varepsilon]$ and between [U] and [o]. For Cantonese speakers, $[\mathrm{I}, \mathrm{U}]$ are on the same level of $[\varepsilon, \mathrm{o}]$, and the difference in $F 2$ is minimal between $[\mathrm{I}]$ and $[\varepsilon]$ and between [U] and [0]. The differences between female speakers of the two languages will also be presented. 
2pSC5. Acoustic characteristics of Korean stops in Korean childdirected speech. Minjung Kim and Carol Stoel-Gammon (Dept. of Speech \& Hearing Sci., Univ. of Washington, 1417 NE 42nd St., Seattle, WA 98105-6246)

A variety of cross-linguistic studies have documented that the acoustic properties of speech addressed to young children include exaggeration of pitch contours and acoustically salient features of phonetic units. It has been suggested that phonetic modifications of child-directed speech facilitate young children's speech perception by providing detailed phonetic information about the target word. While there are several studies reporting vowel modifications in speech to infants (i.e., hyper-articulated vowels), there has been relatively little research about consonant modifications in speech to young children (except for VOT). The present study examines acoustic properties of Korean stops in Korean mothers' speech to their children aged 29 to 38 months $(N=6)$. Korean tense, lax, and aspirated stops are all voiceless in word-initial position, and are perceptually differentiated by several acoustic parameters including VOT, $f 0$ of the following vowel, and the amplitude difference of the first and second harmonics at the voice onset of the following vowel. This study compares values of these parameters in Korean motherese to those in speech to adult Koreans from same speakers. Results focus on the acoustic properties of Korean stops in child-directed speech and how they are modified to help Korean young children learn the three-way phonetic contrast.

2pSC6. Relationship between perceived politeness and spectral characteristics of voice. Mika Ito (Dept. of Linguist., Univ. of Edinburgh, 40 George Square, Edinburgh EH8 9LL, UK, itomika@ ucla.edu)

This study investigates the role of voice quality in perceiving politeness under conditions of varying relative social status among Japanese male speakers. The work focuses on four important methodological issues: experimental control of sociolinguistic aspects, eliciting natural spontaneous speech, obtaining recording quality suitable for voice quality analysis, and assessment of glottal characteristics through the use of non-invasive direct measurements of the speech spectrum. To obtain natural, unscripted utterances, the speech data were collected with a Map Task. This methodology allowed us to study the effect of manipulating relative social status among participants in the same community. We then computed the relative amplitudes of harmonics and formant peaks in spectra obtained from the Map Task recordings. Finally, an experiment was conducted to observe the alignment between acoustic measures and the perceived politeness of the voice samples. The results suggest that listeners' perceptions of politeness are determined by spectral characteristics of speakers, in particular, spectral tilts obtained by computing the difference in amplitude between the first harmonic and the third formant.

2pSC7. Size-constraints on intonation groups in speech: Evidence of an independent syllable-count principle. Annie C. Gilbert and Victor J. Boucher (Univ. of Montreal, C.P. 6128 succ. Centre-ville, Montreal, QC, Canada H3C 3J7)

This poster examines size-limits on intonation $(F 0)$ contours in spontaneous speech and presents the results of an experiment on a syllablecount principle, which is seen to constitute, irrespective of syntax, a factor restricting the length of $F 0$ groups. Studies of various languages indicate a general tendency to restrict stress-groups in speech to four syllables or less. In languages were stress is not lexically coded (e.g., French), syntax is not a sufficient predictor of stress. The object was to determine whether these aspects of stress patterning also apply to tonal groups. Statistics are lacking with respect to the extent of $F 0$ contours in speech. Pitchextracting software was used to analyze the speech of 15 native speakers of French (20 minutes each). Initial results suggest an eight-syllable limit on tonal groups. Based on these statistics an experiment was conducted where $40 \mathrm{Ss}$ read and repeated visually presented sentences containing major syntactic divisions (phrase boundaries) at different locations. There are two central findings: (1) phrase boundaries placed at different points in the sentence did not serve to predict tonal grouping; (2) even when the sentence structure offered the possibility of creating large tonal groups, Ss did not create contours exceeding an eight-syllable limit

2pSC8. Sociological effects on vocal aging: Age related $F \mathbf{0}$ effects in two languages. Kyoko Nagao (Indiana Univ., Memorial Hall 406, 1021 3rd St., Bloomington, IN 47405-7005)

Listeners can estimate the age of a speaker fairly accurately from their speech (Ptacek and Sander, 1966). It is generally considered that this perception is based on physiologically determined aspects of the speech. However, the degree to which it is due to conventional sociolinguistic aspects of speech is unknown. The current study examines the degree to which fundamental frequency $(F 0)$ changes due to advanced aging across two language groups of speakers. It also examines the degree to which the speakers associate these changes with aging in a voice disguising task. Thirty native speakers each of English and Japanese, taken from three age groups, read a target phrase embedded in a carrier sentence in their native language. Each speaker also read the sentence pretending to be 20 -years younger or 20-years older than their own age. Preliminary analysis of eighteen Japanese speakers indicates that the mean and maximum $F 0$ values increase when the speakers pretended to be younger than when they pretended to be older. Some previous studies on age perception, however, suggested that $F 0$ has minor effects on listeners' age estimation. The acoustic results will also be discussed in conjunction with the results of the listeners' age estimation of the speakers.

2pSC9. Cues used for distinguishing African American and European American voices. Erik R. Thomas (Dept. of English, Box 8105, North Carolina State Univ., Raleigh, NC 27695-8105, ethomas@ social.chass.ncsu.edu) and Norman J. Lass (West Virginia Univ., Morgantown, WV 26506-6122)

Past studies have shown that listeners can distinguish most African American and European American voices, but how they do so is poorly understood. Three experiments were designed to investigate this problem. Recordings of African American and European American college students performing various reading tasks were used as the basis for stimuli in all three. In the first experiment, stimuli were subjected to monotonization, lowpass filtering at $660 \mathrm{~Hz}$, and no modification. In the second, stimuli featuring certain ethnically diagnostic vowels and control stimuli were subjected to monotonization, conversion of vowels to schwa, or no modification. In the third, stimuli featuring diagnostic vowels and control stimuli were modified so that the intonation of paired African American and European American speakers was swapped. In all three experiments, African American and European American listeners in North Carolina and European American listeners in West Virginia identified the ethnicity of the speaker of each stimulus. Vowel quality emerged as the most consistent cue for identifications. However, listeners accessed other cues differently for male and female speakers. Breathiness was correlated with identifications of male speakers but not of female speakers. $F 0$-related factors proved more important for female speakers than for male speakers. [Work supported by NSF.]

2pSC10. Effects of aspiration on fundamental frequency in Taiwanese syllables. Yuwen Lai and Allard Jongman (Linguist. Dept., Blake Hall, Univ. of Kansas, Lawrence, KS 66044)

The perturbation effect on vowel fundamental frequency $(F 0)$ by voiceless aspirated and unaspirated prevocalic obstruents is investigated in Taiwanese. It is well known that $F 0$ is significantly higher after voiceless than voiced stops. However, the perturbation effect caused by aspiration has received much less attention. Twenty-eight minimal pairs contrasting in prevocalic aspiration across three different places of articulation from seven tonal categories in Taiwanese were recorded from two male and two female speakers. An acoustic study was conducted on a total of 1120 
syllables. Onset, offset, and mean $F 0$ as well as contours of the tones after the aspirated and unaspirated obstruents were compared. Results indicate that the onset and mean $F 0$ are significantly higher when following aspirated obstruents but no difference was found in offset $F 0$. Tonal contour comparison shows that the $F 0$ raising effect triggered by aspirated obstruents disappears at 50-60\% of the tone. Higher larynx and faster airflow rate are posited as the major factors for the higher $F 0$ after voiceless aspirated obstruents. The present results warrant a reconsideration of the traditional theory of aspiration-induced tonal split according to which a tonal split resulted from the lowering of $F 0$ due to aspiration.

2pSC11. Coarticulatory nasalization in modern Greek: Evidence for a link between coarticulation and syllable structure. Evanthia Diakoumakou (Oakland Univ., Linguist. Dept., Rochester, MI 48309)

An acoustic analysis of the temporal extent of vowel nasalization in the productions of six native speakers of (Standard) Modern Greek showed that the temporal extent of anticipatory vowel nasalization is limited in all contexts, although it is more extensive before tautosyllabic than heterosyllabic nasals. (On average, in stressed syllables, heterosyllabic anticipatory nasalization was $27 \mathrm{~ms}$ long, tautosyllabic anticipatory was 48 ms long, and carryover was $70 \mathrm{~ms}$.) Modern Greek patterns in this aspect with languages like Spanish, Italian, Ikalanga, which also show a tendency toward open syllables, and obstruents are, in general, dispreferred as codas. It is hypothesized that there may be a link between a tendency for open syllables in a language and limited extent of anticipatory nasalization and it is suggested that investigation of the prosodic organization of languages may prove fruitful in determining the factors that lead to crosslanguage coarticulatory differences regarding vowel nasalization.

2pSC12. Examination of voicing onset time during Mandarin tone productions. Yang Chen (Dept. of Speech-Lang. Pathol., Duquesne Univ., 600 Forbes Ave., Pittsburgh, PA 15282) and Manwa Ng (Long Island Univ., Brookville, NY 11548-1300)

The voice onset time (VOT) of stop consonant is defined as the time interval between the release of a stop and the onset of the following vowel (Lisker and Abramson, 1964, 1970). Previous studies indicated that VOT could be used as a perceptual cue for identification of both voicing and place of articulation of stops (Kewley-Port, 1983; Ladefoged, 2001). Abramson (1977) suggested VOT could be interpreted as interrelated acoustic consequences of variation in the relative timing of glottal and oral gestures. This study will attempt to extend the probe on this suggestion by comparing the VOT values among different tones. Mandarin, which is a tone language, where a change in the tone of a syllable leads to a change in meaning, will be used in the study. There are four contrastive tones in Mandarin, each of which is realized by changing the vocal pitch during the course of the syllable production. The proposed study will determine if VOT values for each of the six stop sounds $(/ \mathrm{p}, \mathrm{b}, \mathrm{t}, \mathrm{d}, \mathrm{k}, \mathrm{g} /)$ will vary with the variation of different tones at which each of the following vowel sounds is produced. The interrelationship between VOT and tone production will be discussed.

2pSC13. A perceptual account of dissimilation. Anthony Brasher (Dept. of Linguist., Univ. of Michigan, 4080 Frieze Bldg., 105 S. State St., Ann Arbor, MI 48109-1285)

Ohala [J. J. Ohala, CLS Parasession on Language and Behavior, 178, 203 (1981)] argues that long-distance dissimilation results from hypercorrection; when confronted with words with multiple similar segments, listeners may be confused as to the origin of coarticulated features and attribute cues to one, but not all, segments. The present study evaluates this theory for a sound change involving dissimilation of breathy stops: in sequences of two or more breathy stops, all but the last become modal. If this pattern were reproduced in the laboratory then, when hearing a word with multiple breathy stops, listeners would have trouble assigning the source of the breathy voicing. In this study, Hindi speakers heard two continua of disyllables, where the first syllable varied systematically in the temporal extent of breathy voicing. The continua differed in that the second syllable had a breathy stop in one continuum (dadhak $>$ dhadhak) but a non-breathy stop in the other (dadak $>$ dhadak) $(\mathrm{dh}=$ breathy stop). Initial results for Hindi speakers responding in a four-choice identification task support Ohala's hypothesis. Listeners identified the first syllable reliably when the second syllable is modal, but identified the first syllable inconsistently when the second syllable is breathy.

2pSC14. Pacific northwest vowels: A Seattle neighborhood dialect study. Jennifer K. Ingle, Richard Wright, and Alicia Wassink (Dept. of Linguist., Univ. of Washington, Box 354340, Seattle, WA 98195-4340, merywen@u.washington.edu)

According to current literature a large region encompassing nearly the entire west half of the U.S. belongs to one dialect region referred to as Western, which furthermore, according to Labov et al., “... has developed a characteristic but not unique phonology." [http:// www.ling.upenn.edu/phono_atlas/NationalMap/NationalMap.html] This paper will describe the vowel space of a set of Pacific Northwest American English speakers native to the Ballard neighborhood of Seattle, Wash. based on the acoustical analysis of high-quality Marantz CDR 300 recordings. Characteristics, such as low back merger and $[\mathrm{u}]$ fronting will be compared to findings by other studies. It is hoped that these recordings will contribute to a growing number of corpora of North American English dialects. All participants were born in Seattle and began their residence in Ballard between ages $0-8$. They were recorded in two styles of speech: individually reading repetitions of a word list containing one token each of 10 vowels within carrier phrases, and in casual conversation for $40 \mathrm{~min}$ with a partner matched in age, gender, and social mobility. The goal was to create a compatible data set for comparison with current acoustic studies. $F 1$ and $F 2$ and vowel duration from LPC spectral analysis will be presented.

2pSC15. Breathiness in Indic languages. Christina Esposito, Sameeruddowla Khan, and Alex Hurst (Dept. of Linguist., UCLA, 3125 Campbell Hall Box 951543, LA, CA 90095-1543, esposito@ humnet.ucla.edu)

Previous work on breathiness in Indic languages has focused on the acoustic properties of breathy oral stops in languages like Hindi ([bal] hair versus [bhal] forehead) or Bengali ([baSa] house versus [bhaSa] language). However, breathiness in Indic languages often extends to nasals (e.g., Marathi ([maar] beat versus [mhaar] a caste). It is unclear if languages such as Hindi and Bengali have breathy nasals in addition to breathy oral stops. This study addresses the following questions: (1) Are breathy nasals $(\mathrm{Nh})$ acoustically different from $\mathrm{N}+\mathrm{h}$ sequences, both in languages where they are phonemic and ones where they are not? (2) In sequences of a breathy stop and a modal nasal (e.g., Hindi [udhmi] naughty) where is the breathiness realized, if at all? To answer these questions, audio, aerodynamic, and electroglottographic recordings will be made of Hindi, Bengali, and Marathi speakers. It is hypothesized that acoustically breathy nasals in Hindi and Bengali will not be distinct from sequences of $\mathrm{N}+\mathrm{h}$. We believe that this will also be true for the oral stops. In addition, it is believed that in sequences of breathy oral stop followed by a modal nasal (e.g., $\mathrm{ChN}$ ), the breathiness will be produced on the nasal. 
2pSC16. Low vowels and transparency in Kinande vowel harmony. Bryan Gick, Douglas Pulleyblank (Dept. of Linguist., Univ. of British Columbia, E270-1866 Main Mall, Vancouver, BC, Canada V6T 1Z1, gick@interchange.ubc.ca), Ngessimo Mutaka (Univ. of Yaounde 1, Cameroon), and Fiona Campbell (Univ. of British Columbia, Vancouver, BC, Canada V6T 1Z1)

Transparency - in which a harmony effect passes over a segment without affecting it phonetically or phonologically-has been a controversial concept in previous literature on harmony systems. A typical case of socalled transparency involves cross-height vowel harmony in Kinande, a Bantu language (J.40). Previous accounts have analyzed low vowels in this system as being transparent to harmony [Schlindwein, NELS 17, 551-567 (1987)]. Further, some analysts have considered low vowels theoretically incapable of undergoing tongue root harmony. These claims were tested in a single-subject field study using ultrasound imaging to measure tongue root position in low vowels. Results indicate that (a) advanced versus retracted tongue root position (ATR) is a viable feature for describing the phonological distinction in the vowel system; (b) there is a phonetic difference between low vowels when adjacent to ATR triggering vowels; (c) this distinction in low vowels does not decrease with distance from trigger vowels, suggesting that these vowels are undergoing phonological harmony rather than phonetic assimilation; and finally, (d) the ATR distinction is phonetically categorical in high vowels, but shows crossover in mid and low vowels. Implications for phonological theory and phonetics-phonology interface will be discussed. [Work supported by NSERC and SSHRC.]

2pSC17. The role of psychophysical difference in the discrimination of non-native contrasts. James Harnsberger, Rahul Shrivastav (Dept. Comm. Sci. and Disord., Univ. of Florida, Gainesville, FL 32611), and Mark Skowronski (Univ. of Florida, Gainesville, FL 32611)

Models of cross-language speech perception have shown only limited success in predicting the discriminability of non-native contrasts. These failures may be attributed partly to an inability to quantify the phonetic similarities between non-native speech sounds and between non-native speech sounds and native speech categories. This study represents an attempt to quantify gross psychophysical differences between consonants (e.g., the absolute difference between two consonants as represented in the peripheral auditory system). Two metrics were evaluated with a set of nasal consonant place contrasts from Malayalam. The first focused only on formant transitions at nasal-vowel boundaries (critical cue measure) while the second compared the spectrum of temporal windows spanning the entire length of both syllables that constituted a contrast (whole stimulus measure). The critical cue measure was presumed to have the advantage of incorporating only perceptually relevant information, while the whole stimulus measure was thought to be preferable if the relevant cues were poorly understood and/or were distributed throughout the syllable. The results showed a significant correlation $(r=0.63)$ between the critical cue measure and discrimination scores. The whole stimulus measure showed only a weak correlation $(r=0.35)$ unless a set of outliers (bilabial contrasts) was excluded $(r=0.80)$.

2pSC18. Comparing listener preferences for text-level prosody in English and French. Caroline Smith (Dept. of Linguist., Univ. of New Mexico, MSC 03-2130, Albuquerque, NM 87131-1196)

Cross-language prosodic differences are well-known at the phrasal level and below, and a few studies have shown language-dependent differences in speakers' prosody over a discourse or text [Fon (2002), Smith and Hogan (2003)]. For comparison with the patterns found in production, this study examines listeners' preferences in text-level prosody. Previously-analyzed recordings of one speaker of American English reading aloud an English text, and one speaker of Parisian French reading a comparable French text, were used as a basis. The discourse organization of each text was analyzed by several native speakers who categorized each sentence-to-sentence transition as a Topic Shift, Continuation or Elabora- tion. The original recordings were manipulated in several ways to alter rate, sentence-final lengthening and pause duration, and the manipulated versions were presented to listeners. English listeners liked best those versions where values for rate and lengthening matched the speakers' means for each separate type of topic transition. French listeners preferred the version where each prosodic variable kept the same mean value throughout. These results are supported by acoustic measurements which also point to a more salient role for rhythmic effects at the discourse level in English than in French. [Work supported by NSF grant BCS-9983106.]

2pSC19. Identifying native Cantonese stops: Implication for native speakers perception. Man Gao (Dept. of Linguist., Yale Univ., New Haven, CT 06511)

A series of perception tasks were conducted to probe Cantonese speakers ability to perceive their languages syllable-final unreleased stops. Stimuli consisted of minimal sets of monosyllabic words with contrasting codas produced by a speaker of Hong Kong Cantonese. Twelve Guangzhou Cantonese speakers and five Hong Kong Cantonese speakers performed the perceptual tasks which included: matching stimuli with the corresponding orthographic character; AXB discrimination; and identifying word-final $[\mathrm{p}],[\mathrm{t}],[\mathrm{k}]$ and vowel explicitly. Five native speakers of Mandarin (where final stops are not allowed) also took the AXB and identification tasks. Both Guangzhou and Hong Kong speakers scored high in matching task: 93 and 96 percent correct respectively; for AXB task, subjects from Hong Kong $(93 \%)$ did slightly better than those from Guangzhou (85\%), and Mandarin speakers scored the lowest (78\%). The most interesting findings were in the identification tasks, although subjects from Hong Kong scored the highest (82\%), the accuracy of Guangzhou Cantonese speakers was surprisingly low (49\%) — even worse than Mandarin speakers $(69 \%)$. Explanation for the observed discrepancy between Guangzhou and Hong Kong subjects will be discussed with respect to their different language education as well as their distinct language experience. Implications for phonological awareness will also be discussed.

2pSC20. Processing voiceless vowels in Japanese: Effects of languagespecific phonological knowledge. Naomi Ogasawara (Dept. of Linguist., Univ. of Arizona, Douglass 200E, P.O. Box 210028, Tucson, AZ 85721)

There has been little research on processing allophonic variation in the field of psycholinguistics. This study focuses on processing the voiced/ voiceless allophonic alternation of high vowels in Japanese. Three perception experiments were conducted to explore how listeners parse out vowels with the voicing alternation from other segments in the speech stream and how the different voicing statuses of the vowel affect listeners' word recognition process. The results from the three experiments show that listeners use phonological knowledge of their native language for phoneme processing and for word recognition. However, interactions of the phonological and acoustic effects are observed to be different in each process. The facilitatory phonological effect and the inhibitory acoustic effect cancel out one another in phoneme processing; while in word recognition, the facilitatory phonological effect overrides the inhibitory acoustic effect.

2pSC21. The influence of stress on some acoustic correlates to the stop voicing distinction in French. Nassima Abdelli-Beruh (Dept. of Speech-Lang. Pathol. and Audiol., New York Univ., 719 Broadway Ste. 200, New York, NY 10003 and Dept. of Speech and Hearing Sci., City Univ. of New York, 365 Fifth Ave., New York, NY 10016) and Radhika Aravamudhan (Post-Doc Res. Fellow at Boys Town Natl. Res. Hospital, Omaha, NE)

This study examined how monolingual French speakers produced the stop voicing distinction in stressed and unstressed syllable-initial stops. Syllables were embedded in sentences. Voicing-related differences in du- 
rations of VOT, closure and vowel were calculated and analyzed as a function of stress (stressed on the target syllable, stress on the syllable preceding the target syllable). Percentages of closures with voicing were tallied as function of the voicing category of the stops and the stressed condition. Results from ANOVA showed that the absolute durations were smaller in the unstressed than in the stressed condition. The magnitude of the voicing-conditioned related duration differences in VOT, closure and vowel were also influenced by stress.

2pSC22. The effects of implosives and prenasalized stops on pitch in Shona. Mario E. Chavez-Peon (Dept. of Linguist., Univ. of British Columbia, E-270, 1866 Main Mall, Vancouver, BC, Canada V6T 1Z1 )

It is well known that $F 0$ at vowel onset can be influenced by a preceding consonant. That influence varies significantly across languages and consonant types, and may function as a perceptual signal to consonant manner. It has further been suggested that tone languages may behave differently from non-tone languages in this respect, with a shorter duration of consonantal perturbation [Hombert, Studies in African Linguistics, 1977]. Previous studies include a limited range of consonant types, and too few tone languages to test Hombert's proposal. This study presents the results of an acoustical investigation of the effects of implosives and prenasalized stops on the $F 0$ of a following vowel in Shona, a tone language. It is found that implosives have a similar raising effect on $F 0$ at vowel onset than that of voiceless (aspirated) stops, contrary to expectations based on previous studies [Wright and Shryock, Journal of the Phonetic Association, 1993]. It is also found that prenasalized consonants behave as nasals, having no effect on the $F 0$ of the following vowel, again contrary to expectation [cf. Trithart, Studies in Bantu Tonology, 1976; and Hombert, Studies in Bantu Tonology, 1976]. Finally, duration results do not support Hombert's position regarding tone languages.

2pSC23. Revisiting the Canadian English vowel space. Robert Hagiwara (Linguist. Dept., Univ. of Manitoba, Winnipeg, MB, Canada R3T 5V5)

In order to fill a need for experimental-acoustic baseline measurements of Canadian English vowels, a database is currently being constructed in Winnipeg, Manitoba. The database derives from multiple repetitions of fifteen English vowels (eleven standard monophthongs, syllabic /r/ and three standard diphthongs) in $/ \mathrm{hVd} /$ and $/ \mathrm{hVt} /$ contexts, as spoken by multiple speakers. Frequencies of the first four formants are taken from three timepoints in every vowel token $(25,50$, and $75 \%$ of vowel duration). Preliminary results (from five men and five women) confirm some features characteristic of Canadian English, but call others into question. For instance the merger of low back vowels appears to be complete for these speakers, but the result is a lower-mid and probably rounded vowel rather than the low back unround vowel often described. With these data Canadian Raising can be quantified as an average $200 \mathrm{~Hz}$ or 1.5 Bark downward shift in the frequency of $F 1$ before voiceless /t/. Analysis of the database will lead to a more accurate picture of the Canadian English vowel system, as well as provide a practical and up-to-date point of reference for further phonetic and sociophonetic comparisons.

2pSC24. Concurrent recognition of focus and question in Mandarin. Fang Liu (Univ. of Chicago, Dept. of Linguist., 1010 E. 59th St., Chicago, IL 60637)

F0 has been shown to vary not only with lexical tones but also with focus and sentence type (statement versus question) in Mandarin. This study further investigates whether listeners can detect concurrent focus and sentence type information in speech. 320 statements and unmarked questions with initial, medial, final, or neutral focus produced by two speakers were tested on 11 listeners. Results show that listeners can identify both sentence type and focus in most cases $(89.1 \%$ and $88.7 \%$, respectively), suggesting that $F 0$ variations related to the two functions can be simultaneously perceived. Meanwhile, the lowest rates were found for identifying neutral focus in questions $(71 \%)$ and for identifying statement in sentences with final focus $(78 \%)$. In both cases the confusions seem to arise from the conflicting $F 0$ adjustments by sentence type and focus in the sentence-final position (final $F 0$ being raised for both question and final focus, but not for statement). Overall, the findings not only indicate that $F 0$ can concurrently encode both focus and sentence type in addition to tonal information, but also demonstrate how delicate a balance is maintained between these communicative functions when they have to share the same articulatory/acoustic parameter for their respective manifestations.

TUESDAY AFTERNOON, 17 MAY 2005

GEORGIA B, 1:30 TO 5:00 P.M.

\title{
Session 2pUW
}

\section{Underwater Acoustics: Effects of Environmental Uncertainty}

\author{
David R. Dowling, Chair \\ Dept. of Mechanical Engineering, Univ. of Michigan, 1231 Beal Ave., Ann Arbor, MI 48109-2133
}

\section{Contributed Papers}

$1: 30$

2pUW1. Uncertainty propagation in ocean acoustic waveguides. Steven Finette (Acoust. Div., Naval Res. Lab., Washington, DC 20375)

This presentation discusses a general method for representing uncertainty in numerical models that describe acoustic field propagation within partially specified ocean waveguide environments. The approach treats uncertainty within a probabilistic framework, representing both the sound speed distribution and acoustic field as stochastic processes. In this talk, environmental uncertainty is linked to incomplete knowledge of the vol- ume sound speed field and is directly incorporated into the mathematical formulation for acoustic propagation in a stochastic ocean waveguide. As an example, equations describing the propagation of uncertainty [Finette, J. Acoust. Soc. Am (in press)] are solved analytically for the special case of a point source in a random, but spatially uniform sound speed field bounded by a pressure release surface and a rigid bottom. Results for the first two moments of the acoustic field are calculated using this approach and compared with an independent estimate of the same moments obtained from Monte Carlo based realizations that are computed from a deterministic parabolic wave equation. [Work supported by ONR.] 
2pUW2. Converting sound speed uncertainty to an effective correlated noise term in array processing. Jit Sarkar, Bruce Cornuelle, Philippe Roux, W. S. Hodgkiss, W. A. Kuperman (Scripps Inst. of Oceanogr., UCSD, 9500 Gilman Dr., Mail Code 0238, La Jolla, CA 92093-0238, jit@mpl.ucsd.edu), and Mark Stevenson (NATO SACLANT Undersea Res. Ctr., La Spezia 19138, Italy)

The sensitivity of the received acoustic field at an array from a specific source to perturbations of the sound speed field about a mean value can be computed using a Born approximation formulation involving forward model calculations. When this sensitivity map is appropriately combined with a covariance of the uncertainty of the sound speed perturbations, one obtains the resultant perturbation to the cross spectral density matrix (CSDM) of the acoustic field. This additional term to the mean CSDM can be thought of as a noise term resulting from the uncertainty in the sound speed field. Computations indicate that this additional term is a form of correlated noise that suggests the possibility of mitigating environmental uncertainty with a robust array processing procedure.

\section{2:00}

2pUW3. Rapid acoustic field computations within environmental uncertainty bounds. Kevin D. Heaney (OASIS, Inc., Falls Church, VA 22044)

Oceanographic and geo-acoustic variability in the shallow water can dominate acoustic propagation. Often the environment is critically undersampled, compared to the sensitivity of the full-field propagation model used [such as the Parabolic Equation (PE) model]. For regions with environmental uncertainty (which is known from measurements or models), there will be a Minimum Achievable Uncertainty (MAU) in acoustic propagation modeling (Transmission Loss). Given this MAU, it is proposed that field interpolation techniques be applied to speed up the acoustic propagation computation, consistent with keeping the interpolation errors below the MAU. Techniques for estimating the uncertainty in TL and speeding up the broadband range-dependent computation using the Navy Standard PE model will be presented. For fully 3-D field computations in a range-dependent environment, the speed up is expected to be several orders of magnitude.

\section{2:15}

2pUW4. In-situ estimates of environmental uncertainty on sonar performance prediction. Kevin D. Heaney (OASIS, Inc., Falls Church, VA 22044)

Oceanographic and geo-acoustic variability in the shallow water can dominate acoustic propagation. Sonar performance predictions using archival sound speed and geo-acoustic information do not contain any information about variability or uncertainty in the environment and its likely impact on sonar performance prediction. Using measurements from the NATO Undersea Research Centres BOUNDARY 2003 sea test, an approach is presented that estimates the uncertainty and transfers it through the acoustic propagation and the sonar equation to sonar performance. The sound speed field is characterized by an Empirical Orthogonal Function decomposition of the measured profiles. The geo-acoustic uncertainty is considered to be a normal distribution around the inverted geo-acoustic parameters (from 4 separate inversions). Dynamic ambient noise variability is measured from the array beamformer output. Uncertainty in propagation is quantified in terms of sound speed, geo-acoustic, and ambient noise uncertainty. The uncertainty in sonar performance, due to environmental uncertainty, is then displayed to the operator. [Work supported by the ONR Capturing Uncertainty DRI.]
2pUW5. Performance of mode-based processing in the presence of environmental uncertainty. Lisa Zurk (Elec. \& Comput. Eng., Portland State Univ., P.O. 751, Portland, OR 97207)

A critical limiting factor to the performance of many underwater sonar detection and localization techniques is the typically high degree of uncertainty regarding the ocean environment. Several researchers have proposed mode-based processing methods for Matched Field Processing, depth-dependent pre-filtering, and sub-array processing. Provided that an appropriate modal decomposition can be applied, these methods are believed to provide processing that is less sensitive to environmental mismatch, with the added potential advantage of computational efficiency. In this paper, we attempt to quantify the performance of mode-based processing in uncertain environments, and compare this performance to more classic processing approaches. Results are presented from Monte Carlo simulations of range-dependent environments in which the uncertainty is varied parametrically. An analytical framework for describing the uncertainty is presented and discussed.

\section{$2: 45$}

2pUW6. A high frequency time reversal array deployment in a very shallow water environment. Thomas Folegot (NATO Undersea Res. Ctr., MILOC, Viale San Bartolomeo 400, 19138 La Spezia, Italy), Dominique Clorennec, Jean-Gabriel Minonzio, Julien de Rosny, Claire Prada (Laboratoire Ondes et Acoustique, ESPCI, 75005 Paris, France), Mathias Fink (Laboratoire Ondes et Acoustique, ESPCI, 75005 Paris, France), Sidonie Hibral, and Lothar Berniere (Atlantide, Technopole Brest Iroise, 29238 Brest Cedex 3, France)

A new rigid 24-element acoustic source receiver array in the 10 to 15 $\mathrm{kHz}$ frequency band has recently been developed [Folegot et al., submitted to OCEANS2005-Europe]. Despite of a height of $10 \mathrm{~m}$, it has been specified to be easily deployed from a pier and to allow low cost at-sea experiments with water depth up to $12 \mathrm{~m}$. The array has been deployed in a pool of $50 \mathrm{~m}$ length and $10 \mathrm{~m}$ depth, where calibrated surface plane waves have been generated. The objective of the trial was to undertake a first deployment of the system, to calibrate the instrument and provide some scientific results based on time reversal in a very controlled experimental setup. The Time Reversal Operator has been measured in several configurations. Subsequently, its eigenvectors associated with target reflections have been emitted by the mirror in order to focus separately on each target. The sensitivity of the method against target depth, target type, emission basis, and surface roughness has been investigated. The collected data will help to improve the understanding of environmental factors affecting acoustic propagation in the $10-15 \mathrm{kHz}$ range and will make an important contribution to the development of advanced acoustic communications methods and tomographic capabilities.

\section{3:00-3:15 Break}

\section{3:15}

2pUW7. Improvement of time reversal communications in underwater acoustic channels using adaptive equalizers. Heechun Song, William S. Hodgkiss, William A. Kuperman, Philippe Roux (MPL/ SIO, UCSD, La Jolla, CA 92093), Tuncay Akal (MPL/SIO, UCSD, La Jolla, CA 92093), and Mark Stevenson (NATO Undersea Res. Ctr., La Sapezia, Italy)

The spatial and temporal focusing properties of time reversal methods can be exploited for undersea acoustic communications. Spatial focusing mitigates channel fading and produces a high signal-to-noise ratio at the intended receivers along with a low probability of interception elsewhere. While the temporal focusing (compression) reduces significantly intersymbol interference (ISI), there always is some residual ISI depending upon the number of transmitters and the complexity of the channel. Moreover, a slight change in the environment over the two-way propagation interval introduces additional ISI. Using shallow water experimental data, we dem- 
onstrate that the performance of multiple-input/multiple-output time reversal communications can be improved significantly by cascading the received time series with adaptive equalizers to remove the residual ISI.

\section{3:30}

2pUW8. Broadband source localization using matched correlation processing. Gordon R. Ebbeson, Marie-Noël R. Matthews, Garry J. Heard, Francine Desharnais (DRDC Atlantic, P.O. Box 1012, 9 Grove St., Dartmouth, NS, Canada B2Y 3Z7), and David J. Thomson (Victoria, BC, Canada V9C 4A4)

For many years, model-based signal processing algorithms using Matched Field Processing (MFP) techniques have been analyzed with the goal of improving the capability of passive sonar systems for localizing quiet underwater sources. Recently, researchers at DRDC Atlantic have been investigating Matched Correlation Processing (MCP) as a faster alternative to MFP. In this method, the cross-correlations for a source as measured with a pair of hydrophones in a horizontal array are matched with those generated with a correlation model for many candidate ranges and depths along a candidate bearing. These matches are carried out with a number of hydrophone pairs to form many ambiguity surfaces. The maximum on the average of these surfaces is assumed to yield the best estimate of the source position. By carrying out this procedure over a number of candidate bearings, a full 3-D search for the source location is achieved. Since 2002, a number of localization trials have been carried out east of Nova Scotia, Canada. During those trials, an array was deployed on the sea floor and used to collect acoustic signals from various broadband sources. In this paper, we describe the broadband MCP localization technique and show some localization results from those trials.

\section{$3: 45$}

2pUW9. Broadband continuous wave source localization via pairwise, cochleagram processing. Eva-Marie Nosal and L. Neil Frazer (Univ. of Hawaii at Manoa, 1680 East-West Rd., Post 813, Honolulu, HI 96822)

A pair-wise processor has been developed for the passive localization of broadband continuous-wave underwater sources. The algorithm uses sparse hydrophone arrays and does not require previous knowledge of the source signature. It is applicable in multiple source situations. A spectrogram/cochleagram version of the algorithm has been developed in order to utilize higher frequencies at longer ranges where signal incoherence, and limited computational resources, preclude the use of full waveforms. Simulations demonstrating the robustness of the algorithm with respect to noise and environmental mismatch will be presented, together with initial results from the analysis of humpback whale song recorded at the Pacific Missile Range Facility off Kauai. [Work supported by MHPCC and ONR.]

\section{4:00}

2pUW10. Modeling of forward sector adaptive matched field processing. Jeffrey Dunne (Johns Hopkins Univ. Appl. Phys. Lab., 11100 Johns Hopkins Rd., Laurel, MD 20723, Jeffrey.Dunne@jhuapl.edu)

A modeling study was undertaken to examine the potential benefit of adaptive matched field processing (AMFP) to the forward sector capability of single-line, twin-line, and volumetric arrays. Comparisons are made with conventional MFP (CMFP) and plane-wave beamforming (PWB) in order to assess the degree of ownship noise reduction obtainable and any corresponding improvement to the signal-to-noise ratio (SNR). A minimum variance distortionless response beamformer using dominant mode rejection was implemented, applied to both uniform and distorted array shapes. Significant improvement over CMFP and PWB in tracking and SNR was seen for modeled data in both cases, with the distorted array showing, not surprisingly, better left-right rejection capability. A brief investigation of the effects of environmental mismatch was undertaken in order to assess its potential impact on the value-added of AMFP. [Work was supported by the Defense Advanced Research Projects Agency, Advanced Technology Office.]
2pUW11. An iso-deviant approach for acoustic computations using efficient adaptive gridder for littoral environments. Erik R. Rike and Donald R. DelBalzo (Neptune Sci. Div. of Planning Systems, Inc., 40201 Hwy 190 E, Slidell, LA 70461, delbalzo@neptunesci.com)

Transmission Loss (TL) computations in littoral areas require a dense spatial and azimuthal grid to achieve acceptable accuracy and detail. The computational cost of accurate predictions led to a new concept, OGRES (Objective Grid/Radials using Environmentally-sensitive Selection), which produces sparse, irregular acoustic grids, with controlled accuracy. Recent work to further increase accuracy and efficiency with better metrics and interpolation led to EAGLE (Efficient Adaptive Gridder for Littoral Environments). On each iteration, EAGLE produces grids with approximately constant spatial uncertainty (hence, iso-deviance), yielding predictions with ever-increasing resolution and accuracy. The EAGLE point-selection mechanism is tested using the predictive error metric and 1-D synthetic data-sets created from combinations of simple signal functions (e.g., polynomials, sines, cosines, exponentials), along with white and chromatic noise. The speed, efficiency, fidelity, and iso-deviance of EAGLE are determined for each combination of signal, noise, and interpolator. The results show significant efficiency enhancements compared to uniform grids of the same accuracy. [Work sponsored by ONR under the LADC project.]

\section{$4: 30$}

2pUW12. Adapting a truly nonlinear filter to the ocean acoustic inverse problem. Andrew A. Ganse and Robert I. Odom (Appl. Phys. Lab. (APL-UW), 1013 NE 40th St., Seattle, WA 98105, aganse@apl.washington.edu)

Nonlinear inverse problems including the ocean acoustic problem have been solved by Monte Carlo, locally-linear, and filter based techniques such as the Extended Kalman Filter (EKF). While these techniques do provide statistical information about the solution (e.g., mean and variance), each suffers from inherent limitations in their approach to nonlinear problems. Monte Carlo techniques are expensive to compute and do not contribute to intuitive interpretation of a problem, and locally-linear techniques (including the EKF) are limited by the multimodal objective landscape of nonlinear problems. A truly nonlinear filter, based on recent work in nonlinear tracking, estimates state information for a nonlinear problem in continual measurement updates and is adapted to solving nonlinear inverse problems. Additional terms derived from the system's state PDF are added to the mean and covariance of the solution to address the nonlinearities of the problem, and overall the technique offers improved performance in nonlinear inversion. [Work supported by ONR.]

\section{$4: 45$}

2pUW13. A neural network for mode space source depth estimation. William Lee and Yung Lee (Sci. Applications Intl. Corp., 1710 SAIC Dr., McLean, VA 22102)

A great deal of research in mode space source localization processing exists. One technique proposed by Shang performs mode filtering of the observed pressure field on a vertical line array, using the calculated mode amplitude distributions and phase differences to estimate source location. Ozard et al. proposed a feed forward neural network using input vectors of mode energy distribution for source localization, a dedicated neuron for each possible source location was employed in their study. Instead of employing a dedicated neuron for each possible search location, the interest of this study is to determine an efficient setup of neurons to properly sample the search space. In MATLAB neural network toolbox, the selforganizing-map function learns to classify input vectors according to how they are grouped in the input space, specifically their distribution and topology. We used this function to examine how input vectors of mode energy distribution for signals at different depths are grouped to determine the sampling grids of the search space in depth. We also compared the neural network performance using input vectors of mode amplitude distribution with those using input vectors of mode energy distribution. 


\title{
Meeting of Accredited Standards Committee (ASC) S1 Acoustics
}

\author{
J. P. Seiler, Chair S1 \\ U.S. Department of Labor, Mine Safety and Health Admin., P.O. Box 18233, Bldg. 38, \\ Cochrans Mill Road, Pittsburgh, PA 15236 \\ G. S. K. Wong, Vice Chair S1 \\ Institute for National Measurement Standards, National Research Council, Montreal Road, Bldg. M36, \\ Ottawa, Ontario K1A OR6, Canada
}

\begin{abstract}
Accredited Standards Committee S1 on Acoustics. Working group chairs will report on the status of standards currently under development in the areas of physical acoustics, electroacoustics, sonics, ultrasonics, and underwater sound, etc. Consideration will be given to new standards that might be needed over the next few years. Open discussion of committee reports is encouraged.
\end{abstract}

People interested in attending the meeting of the TAGs for ISO/TC 43 Acoustics and IEC/TC 29 Electroacoustics, take note - those meetings will be held in conjunction with the Standards Plenary meeting at 10:30 a.m. on Tuesday, 17 May 2005.

Scope of S1: Standards, specifications, methods of measurement and test, and terminology in the field of physical acoustics, including architectural acoustics, electroacoustics, sonics and ultrasonics, and underwater sound, but excluding those aspects which pertain to biological safety, tolerance and comfort.

TUESDAY AFTERNOON, 17 MAY 2005

STANLEY ROOM, 3:30 TO 5:00 P.M.

\section{Meeting of Accredited Standards Committee (ASC) S12 Noise}

\author{
R. D. Hellweg, Chair S12 \\ Hewlett Packard Co., Acoustics Lab, MR01-1/P18, 200 Forest Street, Marlborough, MA 01752 \\ R. D. Godfrey, Vice Chair S12 \\ Owens Corning Science \& Technology Center, 2790 Columbus Road, Route 16, Granville, OH 43023-1200
}

\begin{abstract}
Accredited Standards Committee S12 on Noise. Working group chairs will report on the status of noise standards currently under development. Consideration will be given to new standards that might be needed over the next few years. Open discussion of committee reports is encouraged.
\end{abstract}

People interested in attending the meeting of the TAG for ISO/TC 43/SC 1 Noise, take note - that meeting will be held in conjunction with the Standards Plenary meeting at 10:30 a.m. on Tuesday, 17 May 2005.

Scope of S12: Standards, specifications, and terminology in the field of acoustical noise pertaining to methods of measurement, evaluatuion and control, including biological safety, tolerance and comfort and physical acoustics as related to environmental and occupational noise. 